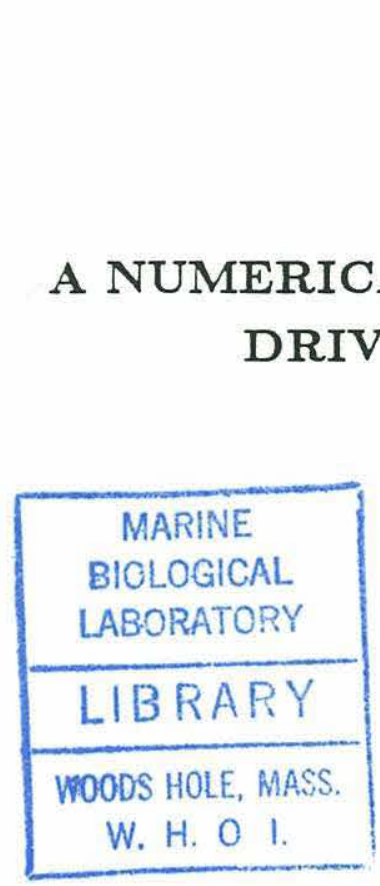

\title{
A NUMERICAL MODEL OF MIXING AND CONVECTION DRIVEN BY SURFACE BUOYANCY FLUX
}

by

MARINE
BIOLOGICAL
LABORATORY

\author{
Xiaoming Wang
}

B.S. Shandong College, China

Submitted in partial fulfillment of the requirements for the degree of

Master of Science

at the

MASSACHUSETTS INSTITUTE OF TECHNOLOGY

and the

WOODS HOLE OCEANOGRAPHIC INSTITUTION

September 1989

(c) Xiaoming Wang 1989

The author hereby grants to MIT and to WHOI permission to reproduce and to distribute copies of this thesis document in whole or in part.

Signature of Author

$$
\begin{array}{r}
\text { Joint Program in Physical Oceanography } \\
\text { Massachusetts Institute of Technology } \\
\text { Woods Hole Oceanographic Institution } \\
\text { August } 7,1989
\end{array}
$$

Certified by

$$
\int / \text { Thesis Supervisor }
$$

Accepted by ...................

Calrl Wunsch Chairman, Joint Committee for Physical Oceanography

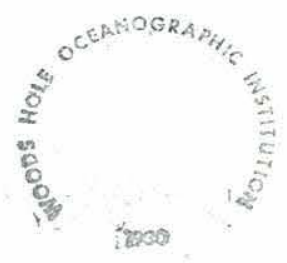




\title{
A NUMERICAL MODEL OF MIXING AND CONVECTION DRIVEN BY SURFACE BUOYANCY FLUX \\ by \\ Xiaoming Wang
}

Submitted in partial fulfillment of the requirements for the degree of Master of Science at the Massachusetts Institute of Technology and the Woods Hole Oceanographic Institution

August 10, 1989

\begin{abstract}
This thesis studies mixing and convection in a rectangular basin driven by a specified heat flux at the surface. A numerical model is constructed for this purpose. The main focus of the study is on the density and circulation structure resulting from the thermal forcing.

In chapter two, a simple vertical one-dimensional model is developed to examine the mixing processes under a given surface heat flux. In order to simulate strong vertical mixing in the region where stratification is unstable, turbulent processes are modeled by a convective overturning parameterization of eddy viscosity and diffusivity. The results show that the density structure is strongly affected by the convective overturning adjustment as surface cooling prevails, and the resulting density field is nearly depth independent.

In chapter three, a more complicated two-dimensional model is constructed to simulate mixing and circulation in a vertical rectangular basin with rigid boundaries. The aspect ratio of the basin ranges from 1 to 0.001 and Rayleigh number from $10^{4}$ to $2 \times 10^{12}$. It is found that the circulation pattern is dominated by these two important numbers. The roles of density overturning and density-momentum overturning mixing are further investigated. The results show that the convective overturning not only homogenizes the density field in the unstably stratified region but also contributes to increase the circulation. A crude scale analysis of the system shows that the characteristics of the density and momentum fields from the analysis agree well with the numerical results.

Thesis Supervisor:

Dr. Glenn R. Flierl, Professor

Massachusetts Institute of Technology
\end{abstract}




\section{Acknowledgments}

I would like to give my sincere thanks to Professor Glenn Flierl, my thesis advisor, for his consistent guidence during the course of completion of this thesis. His creative suggestions can be found in many places of this thesis.

I am also grateful to Dr. Breck Owens for his careful reading and corrections of the thesis draft. His comments not only made this thesis a better presentation, but also deepened my understanding of the results. My thanks also go to Drs. Terry Joyce, Paola Malanotte-Rizzoli and Larry Pratt. Discussions with them in the process of this research were helpful.

It is dedicated with love to Ying and Aileen. 


\section{Contents}

$\begin{array}{lr}\text { Abstract } & 2\end{array}$

$\begin{array}{ll}\text { Acknowledgments } & 3\end{array}$

1 Introduction $\quad 6$

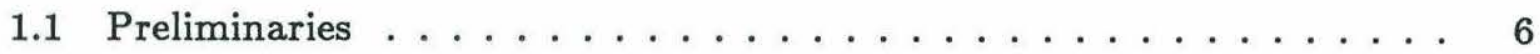

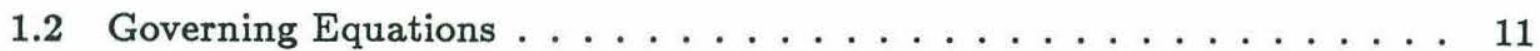

2 Mixing in a One-Dimensional Model $\quad 16$

2.1 Introduction and Governing Equations . . . . . . . . . 16

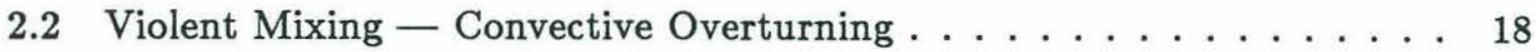

2.3 Numerical Scheme $\ldots \ldots \ldots \ldots \ldots \ldots$. . . . . . . . . . . . 21

2.4 Results and Discussion . . . . . . . . . . . . . . . . 22

2.4.1 Mixing at a Constant Buoyancy Flux . . . . . . . . . 22

2.4 .2 Periodic Forcing $\ldots \ldots \ldots \ldots \ldots \ldots \ldots \ldots$

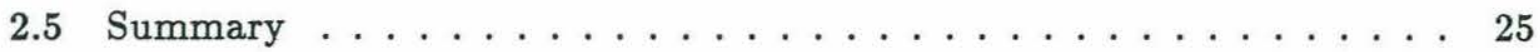


3 Mixing and Circulation in a Two-Dimensional Basin Driven by Surface Buoyancy Flux

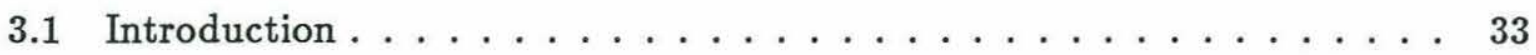

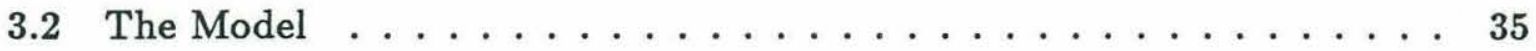

3.3 Numerical Scheme $\ldots \ldots \ldots \ldots \ldots \ldots$

3.4 On the Aspect Ratio of the Basin . . . . . . . . . . . . . 40

3.5 On the Rayleigh Number $\ldots \ldots \ldots \ldots \ldots \ldots$

3.6 The Effects of Mixing $\ldots \ldots \ldots \ldots \ldots \ldots$

3.6.1 Standard Mixing Case $\ldots \ldots \ldots \ldots \ldots \ldots \ldots$

3.6.2 Density Overturning Mixing Case $\ldots \ldots \ldots \ldots \ldots$

3.6.3 Density-Momentum Overturning Mixing $\ldots \ldots \ldots \ldots$. . . 49

3.6.4 Results and Discussion . . . . . . . . . . . . 50

3.7 Analysis on the Dominant Balances of the System . . . . . . . . 54

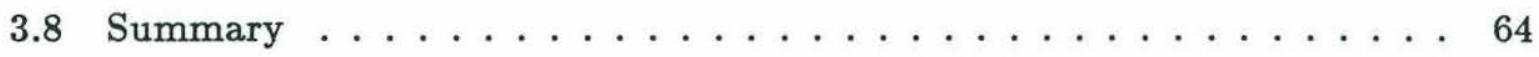

$\begin{array}{lr}\text { Conclusions } & 84\end{array}$

$\begin{array}{lr}\text { References } & 85\end{array}$ 


\section{Chapter 1}

\section{Introduction}

\subsection{Preliminaries}

The ultimate source of energy for the oceanic circulation is energy from the sun. This energy, reaching our planet by solar radiation, is absorbed both by the earth's surface and by its surrounding atmosphere. There are roughly two kinds of forcing driving the oceanic general circulations. One is the direct heating or cooling at the sea surface depending upon the local heat budget; the other is the indirect effect caused by the atmospheric absorption of the solar radiation, essentially leading to wind stresses acting on the sea surface. The oceanic circulation, in general, is an integrated result, coupling these two effects.

For practical reasons, it is customary in oceanography to divide the circulation into different parts according to their forcings. The part driven directly by the exchange of buoyancy at oceanic surface is called the thermohaline circulation. Observations show that the water in the deep ocean is cold and almost uniform in temperature. For example, a fluid column in the tropical region has temperatures ranging from above $20^{\circ} \mathrm{C}$ at the surface to below $2^{\circ} \mathrm{C}$ at $3000 \mathrm{~m}$; while the temperature $9000 \mathrm{~km}$ away in polar ocean is only 0 to $1^{\circ} \mathrm{C}$ at the same depth. This and other observations lead us to the assumed principle of the thermohaline circulation: there must be a general upward advection keeping the abyssal water from warming up by downward heat flux, together 
with the deep circulation it is also responsible for the temperature homogeneity in the deep oceans. Schematically, the thermohaline circulation contains a balance between downward heat diffusion and upward advection over much of the basin and a narrow downward flow of cold water at the coldest edge of the basin. Even though there has been no direct observation of this basinwide upwelling, it is generally accepted by the oceanography community as a basic component of the thermohaline circulation.

The first analytical description of the circulation in an idealized basin was given by Stommel (1950). In his two-page paper Stommel obtained a series solution for temperature and stream function for a two-dimensional advection-diffusion system in a thin basin unevenly heated at the surface. In spite of the fact that the series was only slowly convergent and only two terms were used for the calculation, he was still able to show that the temperature and the velocity fields were asymmetric. This result is non-intuitive and surprising, because there was no asymmetry present in either the basin geometry or the boundary conditions.

Stommel (1962) further investigated a mathematically simplified system. He considered a system of a number of vertical tubes, that were connected at the top with a nonuniform temperature distribution and suspended at the bottom in a fluid reservoir of constant temperature. Upon examination of the equation governing this system at both advective and diffusive limits, Stommel found that the motion was always upward in all but the coldest tube, where the flow went down. Though this model bears no direct resemblance to oceanic problems, it might help to explain the smallness of the sinking regions in the ocean, where the deep and bottom water is formed.

Stone (1968) considered essentially the same set of equations as Stommel (1950) for an axially symmetric system with small aspect ratio. A solution in terms of a series expansion was obtained for small Rayleigh number and again yielded asymmetric patterns of temperature and stream function. 
Laboratory experiments have been performed to verify this thermally driven circulation. One of particular geophysical relevance was performed by Rossby (1965). By imposing a uniform horizontal temperature gradient at the bottom of a small plexiglass tank $\left(24.5 \times 7.5 \times 10 \mathrm{~cm}^{3}\right)$, Rossby successfully showed that a single asymmetric circulation cell exists for a wide range of Rayleigh number. At a low Rayleigh number $\left(1.1 \times 10^{7}\right)$, the thermal boundary near the heated bottom and the upward jet near the warm side wall are relatively wide, and the interior fluid is weakly stratified. As the Rayleigh number is increased (by reducing the viscosity of the fluid), both the heavily stratified bottom layer and the upward jet become thinner, and the interior temperature approaches uniformity. In Rossby's experiment, the interior circulation is downward due to the fact that his tank is heated from the bottom. If the cooling is applied at the surface and the bottom boundary is insulated, there will be upward motion in the interior and sinking next to the coldest wall. Rossby suggested that this asymmetry is a manifestation of the efficient transport of heat by advection relative to diffusion.

As electronic computers became available to the scientific community in the sixties, numerical simulations have brought a new perspection to the problem initially considered by Stommel (1950). This included a number of numerical modelling investigations, for example, the work of Barcilon and Veronis (1965), Somerville (1967), Beardsley and Festa (1972) and Nelken (1987). Barcilon and Veronis considered the same problem as Rossby but with a cosinusoidal bottom temperature distribution and free slip boundary conditions. For a Rayleigh number range of $10^{3}$ to $10^{4}$, they found a weak asymmetric circulation, which was quite independent of the Prandtl number. Somerville (1967) considered this same system of equations with slightly varying boundary conditions. He held the temperature at the upper boundary constant rather than insulated, and considered a basin aspect ratio of 0.1 . The small aspect ratio justified neglecting horizontal diffusion in the governing equations. He adopted spectral analysis and expanded unknowns in double Fourier series. The solutions of both Barcilon 
and Veronis and Somerville exhibit only a slight asymmetry, and neither of them could resolve the boundary layers adequately.

Beardsley and Festa (1972) also studied the same problem as Rossby (1965) with the same noslip boundary condition and linear bottom temperature distribution. They extended the highest Rayleigh number to $3 \times 10^{5}$ and used a unit aspect ratio. Their results showed that the circulation is more asymmetric and the boundary layers are increasingly narrow as the Rayleigh number increases. They also formulated an empirical power law relationship in which the maximum stream function appears to be proportional to $R_{a}^{0.36}$, where $R_{a}$ is the Rayleigh number. Their results show a well defined bottom thermal boundary layer and upward jet, as well as a large homogeneous interior. Figure 1 in their paper illustrates the transition of this system from a diffusion regime at small Rayleigh number to a diffusive-advective regime at large Rayleigh number.

In all the works reviewed above, it is notable that there is always a reversed temperature structure near the base of the jet, i.e., cold heavy fluid overlying warm light ones. This adverse temperature gradient is so large that it is highly unlikely in an oceanic situation. This temperature inversion also appeared in the laboratory experiments of Rossby (1965), but was much weaker than in the numerical simulations. Careful examination of Rossby's results shows that the temperature inversion is most obvious in his small Rayleigh number cases; at large Rayleigh number, this configuration is less obvious and limited only to the small area near the hottest boundary. Considering the fact that Rossby increased the Rayleigh number by reducing the viscosity of the fluid, the existence of the adverse temperature structure in his experiment could be due to the stabilizing effect of high viscosity. One expects that three-dimensional nonhydrostatic effects will limit the possible strength of the inverse gradient. In oceanic situations the Rayleigh number is usually very large, suggesting that the appearence of this adverse temperature structure in the numerical simulations is an artifact, due 
to the lack of proper turbulent (usually three dimensional) mixing scheme in the simulations. To overcome this difficulty, Nelken (1987) introduced convective overturning into the temperature equation by parameterizing the vertical component of the diffusion coefficient $\kappa_{V}$ as a function of stratification. If the stratification is stable, $\kappa_{V}$ is the conventional value used in oceanic mixing problems; if the stratification is unstable, $\kappa_{V}$ is increased to a large value. With the implementation of this turbulent mixing scheme, Nelken's results showed that the unstable temperature structure is replaced by a marginally stable or weakly unstable one, and the isotherms become nearly vertical next to the cooled surface (in his case the basin is unevenly cooled from the top). It is necessary to point out that Nelken adopted an effective Rayleigh number, which was reduced locally where the overturning parameterization was in use. This may be mostly responsible for the weakened circulation in his overturning cases. Nelken's standard cases (non-overturning cases) are remarkably similar to that of Beardsley and Festa (1972), in spite of some important differences in boundary conditions and the omission of the advection terms from the vorticity equation.

Studies in the past four decades have greatly enriched our knowledge about the problem originally initiated by Stommel (1950). In many aspects the problem is still far from being well understood and further investigations are needed. It was started as a problem of direct relevance to large scale oceanography and meteorology, but the investigations until now specified laboratory scales, such as an aspect ratio close to unity in most of the studies and a Rayleigh number many orders of magnitude smaller than in oceans. Those who used a small aspect ratio also had a small Rayleigh number. For example, Stone (1968) solved the small Rayleigh number case, $R_{a}<10^{3}$. In comparison, the Rayleigh number is about $10^{17}$ in the ocean. There are also problems associated with turbulent mixing parameterizations and structure of the boundary layers. These are some of the problems we will address in this study. 
The present work solves the same system as Beardsley and Festa (1972), but with the same free boundary condition and surface flux condition as Nelken (1987). The focus of this work is on the parametric resemblance to the oceans. First the turbulent mixing scheme used by Nelken (1987) is refined to allow smooth spatial changes rather than the original step function. The same mixing scheme is also applied to the momentum field. The basin aspect ratio is varied from 1 to 0.001 . We will focus our attention on the thin basin, typical for phenomena on the oceanic scale. The oceanic values of eddy diffusion coefficients and viscosity will be used throughout the study. Sensitivity to changes in Rayleigh number will be studied, with emphasis on the asymmetry of the circulation and especially the boundary layer structure. The largest Rayleigh number in this work, $2 \times 10^{12}$, surpasses that of any previous theoretical, numerical and laboratory studies. We shall utilize the scale difference in the boundary layers to analyze the balance and the characteristic structure of the circulation.

We should note that our present study is in a non-rotating frame, which severely limits the model applicability to oceanic problems. But, based on what we have achieved here, understanding of the problem within the rotating regime will be our next goal.

\subsection{Governing Equations}

We start with a general dynamical framework commonly used in studying oceanic circulation problems, which includes the momentum equations, the thermodynamic equation and mass conservation equation, and proper boundary conditions.

$$
\begin{aligned}
u_{t}+u u_{x}+v u_{y}+w u_{z}-f v & =-p_{x}+\nu_{H}\left(u_{x x}+u_{y y}\right)+\left(\nu_{V} u_{z}\right)_{z} \\
v_{t}+u v_{x}+v v_{y}+w v_{z}+f u & =-p_{y}+\nu_{H}\left(v_{x x}+v_{y y}\right)+\left(\nu_{V} v_{z}\right)_{z} \\
w_{t}+u w_{x}+v w_{y}+w w_{z} & =-p_{z}+b+\nu_{H}\left(w_{x x}+w_{y y}\right)+\left(\nu_{V} w_{z}\right)_{z} \\
u_{x}+v_{y}+w_{z} & =0
\end{aligned}
$$




$$
b_{t}+u b_{x}+v b_{y}+w b_{z}=\kappa_{H}\left(b_{x x}+b_{y y}\right)+\left(\kappa_{V} b_{z}\right)_{z}
$$

where $x, y$ and $z$ are axes with east, north and upward directions, $u, v$ and $w$ are the velocity components in these directions, respectively, $p$ denotes pressure, $b=-g \rho / \rho_{0}$ buoyancy, $g$ the gravitational acceleration, $\rho=\rho_{0}\left[-\alpha\left(T-T_{0}\right)+\beta\left(S-S_{0}\right)\right]$ density variation, $T$ temperature, $S$ salinity, $\rho_{0}, T_{0}$ and $S_{0}$ are reference density, temperature and salinity, respectively. $\alpha=\left(1 / \rho_{0}\right) d \rho / d T$ is the thermal expansion coefficient of sea water and $\beta=\left(1 / \rho_{0}\right) d \rho / d S$ the correspondng coefficient for salinity. $\nu_{H}$ and $\nu_{V}$ are the horizontal and vertical components of the kinematic viscosity, and $\kappa_{H}$ and $\kappa_{V}$ the horizontal and vertical components of diffusivity. The vertical components of both viscosity and diffusivity may be functions of time and space and will be further discussed later in this thesis. The Boussinesq approximation was already used to obtain equations (1.1).

Equations (1.1) are the fully nonlinear primitive equations. It is a formidable task to find a solution for this system together with the realistic initial and boundary conditions, even numerically. Fortunately, limitations of various kinds often apply, so that we can simplify the system. Assume that the fluid is confined in a channel which is infinite in the $x$-direction and rectangular in $y-z$ cross section. Attention is restricted to only those problems that display no $x$ dependency. Then in (1.1) all $x$ derivatives drop out, and the new continuity equation (1.1d) defines a streamfunction $\psi$, such that

$$
v=-\psi_{z}, \quad w=\psi_{y}
$$

Making use of this streamfunction and cross differentiating (1.1b) and (1.1c) to eliminate pressure, we can reduce the system to the following three equations,

$$
\begin{aligned}
u_{t}+J(\psi, u)+f \psi & =\nu_{H} u_{y y}+\left(\nu_{V} u_{z}\right)_{z} \\
\left(\nabla^{2} \psi\right)_{t}+J\left(\psi, \nabla^{2} \psi\right)-f u_{z} & =b_{y}+\nu_{H}\left(\nabla^{2} \psi\right)_{y y}+\frac{\partial}{\partial z}\left[\nabla \cdot\left(\nu_{V} \nabla \psi_{z}\right)\right] \\
b_{t}+J(\psi, b) & =\kappa_{H} b_{y y}+\left(\kappa_{V} b_{z}\right)_{z}
\end{aligned}
$$


where $\nabla=\hat{\mathbf{y}} \partial / \partial y+\hat{\mathbf{z}} \partial / \partial z, \nabla^{2}=\partial^{2} / \partial y^{2}+\partial^{2} / \partial z^{2}$, and $J(\psi)=,\psi_{y} \partial / \partial z-\psi_{z} \partial / \partial y$. The system in (1.2) is still nonlinear but the unknowns have been reduced from five in (1.1) to three in (1.2). The simplicity of equations (1.2) over (1.1) is obvious. For the geometry chosen here, if none of the boundary conditions and forcing exhibit $x$ dependency, and no turbulence is involved, the solution to equations (1.2) are exactly the same as that of (1.1). In any real oceanic situation, the boundary conditions, topography and forcing exhibit variations in three dimensions, the evolution of instabilities may result in the onset of three dimensional turbulence, the momentum and density fields will no longer be two dimensional. In this sense equations (1.2) can only be regarded as an idealized two-dimensional approximation of (1.1).

The boundary conditions needed to solve (1.2) are the specification of heat flux or temperature on all boundaries, as well as velocities or stresses. They can be of the first kind (Dirichlet boundary condition, such as specifying the streamfunction value) or of the second kind (Neumann boundary condition, such as specifying heat flux), or a mixture of the two.

For convenience, equations (1.2) will be nondimensionalized by basic scales of the system. If dimensionless quantities are denoted by primes, we introduce following scales,

$$
\begin{aligned}
y & =L y^{\prime}, \quad z=D z^{\prime}, \quad f=f_{0} f^{\prime} \\
\psi & =\Psi \psi^{\prime}, \quad u=U u^{\prime}, \quad b=B b^{\prime} \\
\kappa_{V} & =\kappa \kappa_{V}^{\prime}, \quad \nu_{V}=\nu \nu_{V}^{\prime}
\end{aligned}
$$

In this work we mainly focus on mixing and motion driven by differential heating and cooling; it is physically consistent to use a diffusive time scale

$$
t=\frac{D^{2}}{\kappa} t^{\prime}
$$


It is convenient to introduce some commonly used dimensionless numbers,

$$
\begin{aligned}
\lambda & =\frac{D}{L}, \quad \epsilon=\frac{U}{f_{0} L}, \quad F=\frac{f_{0}^{2} L^{2}}{g D} \\
\sigma & =\frac{\nu}{\kappa}, \quad \delta=\frac{\Psi D}{\nu L} \\
\bar{\nu}_{H} & =\frac{\nu_{H}}{\nu} \frac{D^{2}}{L^{2}}, \quad \bar{\kappa}_{H}=\frac{\kappa_{H}}{\kappa} \frac{D^{2}}{L^{2}} \\
E_{H} & =\frac{\nu_{H}}{f_{0} L^{2}}, \quad E_{V}=\frac{\nu}{f_{0} D^{2}}
\end{aligned}
$$

where $\lambda$ is the aspect ratio, $\epsilon$ the Rossby number, $F$ the Froude number, $\sigma$ the Prandtl number, $\delta$ the Reynolds number, and $E_{H}$ and $E_{V}$ the horizontal and vertical Ekman numbers. $\bar{\nu}_{H}$ and $\bar{\kappa}_{H}$ are the dimensionless horizontal vicosity and diffusivity. Substituting the above dimensionless quantities and numbers in the equation (1.2) and rewriting it in dimensionless form, we obtain

$$
\begin{aligned}
\frac{1}{\sigma} u_{t}+\delta J(\psi, u)+\frac{\delta}{\epsilon} f \psi_{z} & =\bar{\nu}_{H} u_{y y}+\left(\nu_{V} u_{z}\right)_{z} \\
\frac{E_{V}^{2}}{\sigma}\left(\nabla^{2} \psi\right)_{t}+\delta E_{V}^{2} J\left(\psi, \nabla^{2} \psi\right)-\frac{\epsilon}{\delta} f u_{z} & = \\
\frac{B}{g \delta F} b_{y}+E_{H} E_{V}\left(\nabla^{2} \psi\right)_{y y} & +E_{V}^{2} \frac{\partial}{\partial z}\left[\nabla \cdot\left(\nu_{V} \nabla \psi_{z}\right)\right] \\
b_{t}+\delta \sigma J(\psi, b) & =\bar{\kappa}_{H} b_{y y}+\left(\kappa_{V} b_{z}\right)_{z}
\end{aligned}
$$

where $\nabla=\hat{\mathbf{y}} \lambda \partial / \partial y+\hat{\mathbf{z}} \partial / \partial z$ is the dimensionless gradient operator and $\nabla^{2}=\lambda^{2} \partial^{2} / \partial y^{2}+$ $\partial^{2} / \partial z^{2}$ is the dimensionless Laplace operator. Now in (1.4) all the dependent and independent variables are of order one and are dimensionless. For the sake of neatness, we have omitted primes on the dimensionless quantities. Since $\kappa_{V}$ and $\nu_{V}$ will have spatial variations they are kept inside the brackets of the vertical derivative.

In (1.3) not all of the nine parameters are independent. From the well known hypothesis of the maintenance of oceanic thermocline, we know that in most of the ocean there is a slow upwelling that balances the natural downward diffusion of heat. This suggests that in the advection-diffusion equation (1.4c), $\delta \sigma=O(1)$. For simplicity we let $\delta \sigma=1$ in the equation. This, in turn, gives a scale for streamfunction, $\Psi=$ 
$\kappa L / D=\kappa / \lambda$. This shows that the thermally induced motion depends on the diffusive nature of the fluid and the aspect ratio of the basin.

If equation (1.4b) is divided by $E_{V}^{2}$, and we define one important parameter,

$$
R_{a}=\frac{B D^{3}}{\nu \kappa},
$$

the Rayleigh number, a ratio of the destabilizing buoyancy force to the stabilizing diffusive forces, we can rewrite (1.4) to give,

$$
\begin{aligned}
\frac{1}{\sigma}\left[u_{t}+J(\psi, u)+\frac{1}{\epsilon} f \psi_{z}\right] & =\bar{\nu}_{H} u_{y y}+\left(\nu_{V} u_{z}\right)_{z} \\
\frac{1}{\sigma}\left[\left(\nabla^{2} \psi\right)_{t}+J\left(\psi, \nabla^{2} \psi\right)\right]-\frac{\epsilon}{\delta E_{V}^{2}} f u_{z} & = \\
R_{a} \lambda^{2} b_{y}+\bar{\nu}_{H}\left(\nabla^{2} \psi\right)_{y y} & +\frac{\partial}{\partial z}\left[\nabla \cdot\left(\nu_{V} \nabla \psi_{z}\right)\right] \\
b_{t}+J(\psi, b) & =\bar{\kappa}_{H} b_{y y}+\left(\kappa_{V} b_{z}\right)_{z}
\end{aligned}
$$

In equation (1.5) the terms involving the effects of rotation are $f \psi_{z}$ and $f u_{z}$ only. If one wants to study this system in the absence of rotation, one can simply set $f=0$ in equations (1.5). If only thermally driven motion is considered and $f=0$, there is no internal or external forcing to support the zonal flow field; thus the first equation can be dropped from (1.5). If we fix $\sigma$ in this simplified system (the system is quite independent of $\sigma$ according to Barcilon and Veronis, 1965), there is only one parameter that can be freely chosen, i.e., the Rayleigh number. The importance of this parameter and the behavior of the system when the number varies will be discussed extensively later in chapter three. 


\section{Chapter 2}

\section{Mixing in a One-Dimensional Model}

\subsection{Introduction and Governing Equations}

To investigate the mixing processes induced by thermal forcing we begin with the simplest case, one-dimensional vertical mixing. Suppose that equations (1.4) exhibit no horizontal dependence and the Coriolis force is neglected, so that only vertical mixing of temperature and dissipation of momentum is involved. We will examine the response of this idealized system to external thermal forcing. The shortcoming of this approximation is obvious, since a thermally induced advection field is absent in any one-dimensional model. In a one-dimensional system reduced from (1.4) the buoyancy field is decoupled from the momentum field. The pre-existing momentum field will simply die out if no internal sources or external forces are present to balance dissipation. The physical interpretation of the results of this mathematically simple problem makes it worthwhile for studying oceanic mixing cases.

As long as there is a net heat flux into the system, there will be no absolutely steady state reached. But for periodic thermal forcing, there will be a periodic steady state established after some time. When the fluid is cooled from the surface (or heated from the bottom), a gravitationally unstable stratification is found (figure (2.1a)), with heavy fluid on top of light fluid. To avoid this highly unrealistic situation, following Nelken (1987), we use a parameterization of convective overturning to simulate the 
violent vertical mixing that would occur due to the unstable density stratification. The new results show that it efficiently smooths out the unrealistic density gradient (figure (2.1c)).

The governing equations reduced from equations (1.4) are the decoupled buoyancy (temperature) and vorticity equations,

$$
\begin{aligned}
b_{t} & =\left(\kappa b_{z}\right)_{z} \\
\zeta_{t} & =(\nu \zeta)_{z z}
\end{aligned}
$$

where vorticity $\zeta=\psi_{z z}, \kappa$ and $\nu$ may be functions of both $t$ and $z$. The boundary conditions are a given flux $Q$ at surface $z=0$ and no flux at bottom $z=-1$, vorticity and streamfunctions are zero on both surface and bottom boundaries. Because in (2.1) the buoyancy and vorticity fields are not related, the thermal forcing will only modify the buoyancy field through the vertical mixing mechanism.

Integrating (2.1a) from bottom to surface gives

$$
\frac{\partial}{\partial t} \int_{-1}^{0} b d z=\left.\kappa b_{z}\right|_{z=0}
$$

so that the rate of change of total buoyancy in the fluid column is proportional to the buoyancy flux at the boundary. The way that fluid mixes in the column and the resulting vertical structure are obviously dependent upon the way that mixing is parameterized. As for the vorticity field, the diffusion and viscous dissipation will finally diminish it to zero, since no generation process exists to supply vorticity to counteract dissipation.

Before looking for a solution to (2.1), we will first investigate some obvious characteristics of the solution. The solution for $b$ in (2.1) has a gravitationally unstable structure if buoyancy is lost at surface and a stable structure if buoyancy is gained at surface. In the latter case the gravity force stabilizes the fluid column, the mixing is dominated by slow diffusion. The resulting field is surface intensified, since the slowness 
of diffusion is unable to carry the heat effectively to the region below and thus builds up a strong gradient near the surface. What we are really interested in is the former case where a gravitationally unstable stratification makes the mixing process totally different from the latter one. As mentioned before, the idea of mixing by convective overturning is introduced, and the resulting buoyancy field is marginally stable to weakly unstable.

\subsection{Violent Mixing - Convective Overturning}

It is known that whenever a heavy fluid lies on top of lighter ones, the fluid will overturn and rapidly mix to form a marginally stable column. This is almost always a three-dimensional process and therefore must be parameterized in a two-dimensional model. In theory this may be done by imposing large mixing coefficient for the fluid where stratification is unstable (Bryan and Cox, 1968; Killworth, 1976). That is, equation (2.1a) is solved with a vertical diffusion coefficient that depends on $b_{z}$,

$$
b_{t}=\left(\kappa b_{z}\right)_{z}
$$

where

$$
\kappa= \begin{cases}\kappa^{+}, & b_{z} \geq 0 \\ \kappa^{-}, & b_{z}<0\end{cases}
$$

where $\kappa^{+}=O(1)$ and $\kappa^{-} \gg O(1)$. Both $\kappa^{+}$and $\kappa^{-}$are positive and constant. When buoyancy is lost from the fluid column, the above parameterization of vertical mixing results in an almost uniform buoyancy structure in the column. The same parameterization was used by Nelken (1987) to study water mass formation and thermally driven circulation. Letting $\kappa^{-}$go to infinity is equivalent to averaging $b$ within the depth over the overturning region (region $b_{z}<0$ ). Theoretically a step function $\kappa$ as defined above may cause a sudden jump in right hand side of equation (2.3) at $b_{z}=0$. It is fortunate that in equation (2.3) this term is multiplied by $b_{z}$ itself, which keeps the term $\left(\kappa_{z} b_{z}\right)$ finite. Thus a step function $\kappa$ still leads to a continuous, smooth buoyancy field. Unfortunately when momentum overturning mixing is considered with the same 
kind of parameterization for $\nu$ as a function of $b_{z}$, it results in a singular behavior of equation (2.1b) at $b_{z}=0$. This is a problem when we solve both (2.3) and (2.1b) with overturning mixing parameterization.

An obvious choice is to make $\kappa$ a continuous function of $b_{z}$, which retains the basic idea of overturning mixing but remove the singularity at $b_{z}=0$. After many test cases the form of $\kappa$ employed in this study was chosen to be

$$
\kappa=\kappa^{+}+\kappa^{-} b_{z} \tanh \left(\gamma b_{z}\right) H\left(-b_{z}\right)\left|b_{z}\right| /\left|b_{z}\right|_{\max }
$$

where $H(x)=0$ for $x \leq 0$ and 1 for $x>0$ and $\gamma$ is a constant. The second term on the right hand side of (2.4) is switched on only when $b_{z}<0$, that is, for convective overturning. Later in this thesis when we refer to nonoverturning mixing, it means that $\kappa^{+}=1$ and $\kappa^{-}=0$. Otherwise $\kappa^{+}=1$ and $\kappa^{-}$is a given large constant. When momentum overturning is included, $\nu$ is parameterized exactly as $\kappa$.

Parameterizing mixing in the above ways, we enter a nonlinear regime rather than the original simple linear system. The mixing coefficient is no longer a known value for the fluid but a function of stratification.

When the fluid is cooled constantly from above, the stratification may reach a point that it is unstable for the whole fluid column. This enables us to use a large and constant value for $\kappa$ and thus to solve $b$ by expanding it in powers of $1 / \kappa$

$$
b=b_{0}+(1 / \kappa) b_{1}+(1 / \kappa)^{2} b_{2}+\ldots
$$

Substituting into (2.3) and grouping terms in powers of $1 / \kappa$, the zero and first order balances are

$$
\begin{aligned}
b_{0 z z} & =0 \\
b_{0 t} & =b_{1 z z}
\end{aligned}
$$

the boundary conditions at the surface and bottom give

$$
\kappa b_{0 z}+b_{1 z}=Q
$$




$$
\kappa b_{0 z}+b_{1 z}=0
$$

where $Q=O(1)$, we get $b_{0 z}=0, b_{1 z}=Q$ at $z=0$, and $b_{0 z}=b_{1 z}=0$ at $z=-1$. Integrating the zero order balance equation and using the boundary conditions, we obtain

$$
b_{0}=c_{1}(t)
$$

while the first order balance gives

$$
b_{1}=0.5 c_{1 t} z^{2}+c_{2}(t) z+c_{3}(t) .
$$

The boundary conditions give $c_{2}=Q$ and $c_{1}=\int Q d t+c_{4}$, where $c_{4}$ is an integration constant and can be combined into $c_{3}$. If at $t=0, b=0$, then $c_{3}=0$. Thus to the first order of $1 / \kappa$, the solution is

$$
b(z, t)=\int Q d t+(1 / \kappa) Q z(1+z / 2)
$$

The dependency of $b$ on $\kappa$ can be seen qualitatively even in this very approximate solution. When $\kappa$ is infinitely large, corresponding to infinite mixing, the mixing process becomes instantaneous, and the buoyancy in the whole fluid column is uniform and changes at a rate equal to the surface flux. The fluid column, acting as a slab, has no vertical structure. When $\kappa$ is finite, there is a weakly unstable vertical structure in addition to the uniformly changing background. The larger $\kappa$, the weaker the vertical structure. For this constant heat flux case, the vertical gradient of $b$ is approximately linear.

When $\kappa$ and $\nu$ are parameterized in the same way, equation (2.1b) still differs from equation (2.1a). As already discussed previously, (2.1b) involves a singularity at $b_{z}=0$. By using (2.4), we may avoid this singular point, but the behavior of the solution for $\varsigma$ may still not be smooth around $b_{z}=0$, since it involves the second derivative with respect to $z$. Our goal is to mix the momentum by the same overturning mechanism as we mix the buoyancy, so that as the buoyancy field varies smoothly, $\nu$ 
will be continuous and reasonably smooth; thus the resulting momentum field will also be regular and smooth.

\subsection{Numerical Scheme}

In this section we solve equations (2.1) numerically given initial and boundary conditions and $\kappa, \nu$ parameterization as in (2.4). The finite difference form of (2.1) using forward time and central space difference becomes

$$
\begin{gathered}
\frac{b(j, n+1)-b(j, n)}{\Delta t}=\frac{\kappa(j+1)[b(j+1, n)-b(j, n)]-\kappa(j)[b(j, n)-b(j-1, n)]}{\Delta z^{2}} \\
\frac{\varsigma(j, n+1)-\varsigma(j, n)}{\Delta t}=\frac{\nu(j+1) \zeta(j+1, n)-2 \nu(j) \zeta(j, n)+\nu(j-1) \zeta(j-1, n)}{\Delta z^{2}}
\end{gathered}
$$

and

$$
\varsigma(j, n)=\frac{\psi(j+1, n)-2 \psi(j, n)+\psi(j-1, n)}{\Delta z^{2}},
$$

where $t=n \Delta t$ and $z=j \Delta z$. The grid configuration is staggered, so that $\zeta(j, n)$ and $\psi(j, n)$ are located at the same point and $b(j, n)$ is in between $\zeta(j, n)$ and $\zeta(j-1, n)$. The diffusion coefficients $\kappa$ and $\nu$ are evaluated in between $b(j+1, n)$ and $b(j, n)$, i.e., on the $\varsigma(n, j)$ and $\psi(n, j)$ grid point. In such a grid system, Dirichlet boundary conditions for vorticity and streamfunction can be directly applied at the surface and bottom grids, while each control volume (viewed as a rectangle of unit width and length $\Delta z$ ) bounded by two neighboring $\psi$ (or $\zeta$ ) points has buoyancy $b$.

The Von Neumann stability analysis (Roache, 1972, p42) gives the upper bound of the size of the time step that guarantees a convergent solution to (2.1)

$$
\Delta t<\frac{\Delta z^{2}}{\kappa}
$$

When the time step is chosen to meet this criterion, the information will not travel more than one grid length at each step thus allowing the field to adjust itself one point 
per time step. Thus, one artifact of this discrete time difference is that the speed of information propagation is limited.

\subsection{Results and Discussion}

\subsubsection{Mixing at a Constant Buoyancy Flux}

For a constant surface buoyancy loss, we will study solutions to (2.1) for different parameter ranges. With $Q=-1, \kappa^{+}=1, \kappa^{-}=0$, no momentum exchange occurs at all boundaries. Initially we have a uniform buoyancy field and a Gaussian vorticity field (symmetric about mid depth). The results, after $6.4 \times 10^{5}$ time steps, are shown in figure $(2.1 \mathrm{a}, \mathrm{b})$. The horizontal and vertical axes are time and depth, respectively, the contours are buoyancy in (2.1a) and streamfunction in (2.1b). The most prominant feature seen here is the very unstable structure of the buoyancy field. The cooling at the surface moves downward slowly by the conventional mixing process, even though the structure is statically unstable. It causes a reversed density structure, i.e., the heavy fluid lying on top of the lighter ones.

The results of a similar case but with $\kappa^{-}=100$ are shown in figure $(2.1 \mathrm{c}, \mathrm{d})$. The buoyancy contours become vertical as overturning mixing tends to smooth out the negative vertical buoyancy gradient. The cooling persists all the way from the surface to the bottom, with the maximum flux at the top. The buoyancy structure is weakly depth dependent (inversely proportional to $\kappa^{-}$). Comparing figure (2.1a) with (2.1c), one can immediatly recognize that the later case is more physically realistic and acceptable. From (2.1c) we can see that the tilting of the b-contours stays almost unchanged as time goes by, indicating that even though the total buoyancy in the column is varying, the flux across a given depth is fixed. This flux, maximum at surface, decreases with depth. This can also be deduced from the parameterization of $\kappa$. Near the surface, $\kappa$ is large, and thus a larger flux. $\kappa$ decreases with depth since less flux is needed to 
adjust the buoyancy field below the surface, thus $\left|b_{z}\right|$ also decreases with depth. This is clearly seen in figure (2.3), where we plot buoyancy and vertical buoyancy gradient as functions of depth (vertical axis) at thirteen different times (horizontal axis) for both nonoverturning and overturning cases. We observe a continuous increase of $b$ and a continuous decrease of $\left|b_{z}\right|$ with depth in both cases. The magnitude of the changes with depth are much larger in $(2.3 \mathrm{a}, \mathrm{b})$, where $\kappa^{-}=0$, while the changes in $(2.3 \mathrm{c}, \mathrm{d})$ are hardly seen (close to vertical lines), where $\kappa^{-}=100$. The convective overturning mixing gives a more realistic buoyancy structure than the nonoverturning case does.

We have a different situation when solving the momentum equation. Since no boundary flux and internal sources of momentum exist, the initial flow field simply dies out due to viscous dissipation. We expect that this dissipation process is slow if no overturning of momentum is involved and fast if overturning of momentum is involved. Figure $(2.1 \mathrm{~b}, \mathrm{~d})$ shows our intuition is correct. One can choose to overturn only buoyancy or both buoyancy and momentum; the different combination of parameterization will be explored later in chapter three of this thesis. Here only two choices are necessary, since $b$ and $\zeta$ are dynamically unrelated.

In the momentum overturning case, it is essential that dissipation of momentum be smooth and continuous. Since later in chapter three the momentum field is related to buoyancy field through advection and baroclinic generation of vorticity, it is important for the momentum field not to be singular. Figure (2.2) is an enlarged version of figure (2.1) for the first $1 / 6$ th of the total time steps. It can be seen from figure $(2.2 \mathrm{~b}, \mathrm{~d})$ that no appreciable singular behavior of the streamfunction exists. Further plots of temperature (buoyancy), vorticity and streamfunction values at three different depths all show smooth changes of these quantities with time (Figure (2.4) and (2.5)). A more detailed version of figure (2.5) is shown in (2.6) for the first $1 / 6$ th time steps. Particular attention is paid to both vorticity and streamfunction. They all approach zero smoothly within a relatively short period of time because the fluid column is always subject to 
the convective overturning condition. If we examine figure $(2.2 \mathrm{~b}$ and $\mathrm{d})$ very carefully, we can see that momentum dissipation in figure $(2.2 \mathrm{~b})$ is uniform and symmetric, while in (2.2d) it is slightly asymmetric, due to the buoyancy structure and the varying of $\kappa$ as a function of $b$. When a surface momentum flux is also imposed, we see that it will be quickly mixed downward and homogenized over the depth of the column.

\subsubsection{Periodic Forcing}

Results for the case with a periodic surface flux of buoyancy are given in figure (2.7). The forcing is simply a sinusoidal, time dependent buoyancy flux at the surface, $Q=\sin \omega t$. Since our main focus is on the model behavior, we do not require the forcing function to resemble the actual seasonal cycle. Figures $(2.7 \mathrm{a}$ and $\mathrm{b})$ show buoyancy and streamfunction contours as functions of time and depth for the first five forcing periods. As anticipated we see that a periodic buoyancy field develops quickly since overturning mixing is involved; only one period is needed compared to three periods for the nonoverturning mixing case. The buoyancy structure is surface trapped, with a thermocline developed during the heating phase. It penetrates only to a small depth. The onset of cooling quickly destroys it and overturns the dense water at the surface with the light ones below. At the end of the cooling phase, the net flux into the system is zero, and the whole fluid column is almost neutrally stratified. Thus the periodic heating and cooling events look like isolated pulses, separated by cooling and heating phases.

Figure (2.7c) shows the buoyancy vs. time at three different depths. The amplitudes at different depths show the surface trapping effect. The gentle slope of the curves is associated with heating and steep slope with cooling, while the flat troughs indicate that violent mixing occurs during the cooling phase and homogenizes the water column. The absence of flat troughs in curve $c$ is due to the fact that it is right 
below the surface and responds to either heating or cooling at almost the same rate and amplitude.

Momentum mixing, parameterized exactly as buoyancy mixing, gives fast but smooth dissipation of the vorticity and decay of the streamfunction $(2.7 \mathrm{~b})$. Stream functions at three different depths are plotted in figure $(2.7 \mathrm{~d})$ for the first forcing period. They approach zero within the first cooling phase, but a little slower than in the constant cooling case shown in (2.6d), due to a decrease of mixing as the surface flux decreases with time.

\subsection{Summary}

When the water column is cooled from the surface, the convective overturning mixing process prevails, the density is nearly uniform for the whole column. The small deviation from uniformity is due to the fact we used a finite vertical diffusion coefficient in (2.4). If $\kappa$ is infinitely large and thus $\Delta t$ is infinitely small according to the Von Neumann stability criterion (2.5), the contours in (2.1c) will approach vertical. This practicality limits us to a moderate diffusion coefficient, such as $\kappa^{-}=100$. Our

numerical results with large $\kappa^{-}$did not significantly change from those for moderate $\kappa^{-}$, except more CPU time was consumed, so we decided to use $\kappa^{-}=100$. Later in chapter three, the same parameterization and value for $\kappa$ will be used in a two dimensional advection-diffusion model to examine the buoyancy driven circulation.

The simulation with periodic forcing shows that a periodic steady response of the buoyancy structure can be achieved with the overturning mixing parameterization. The variability of the density field is largest near the surface. The cooling phase, after eroding the thermocline generated by surface heating, produces a nearly uniform water column with weak vertical gradients. Then the heating proceeds to reconstruct the thermocline and the process repeats. 


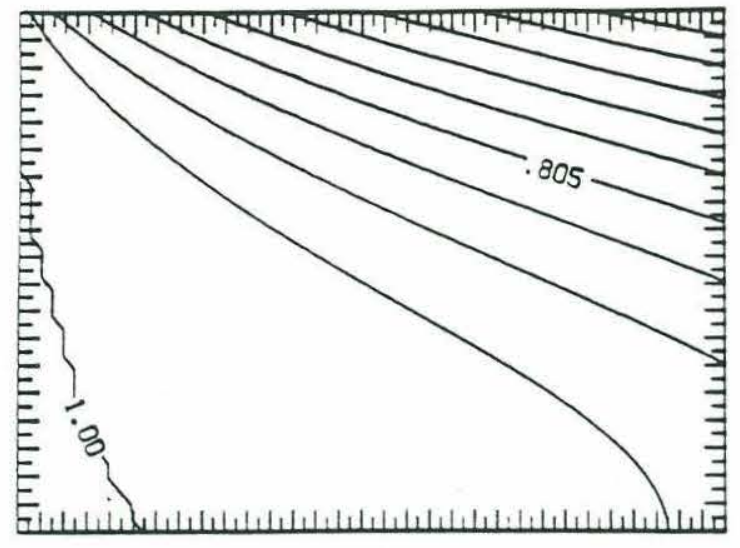

(a)

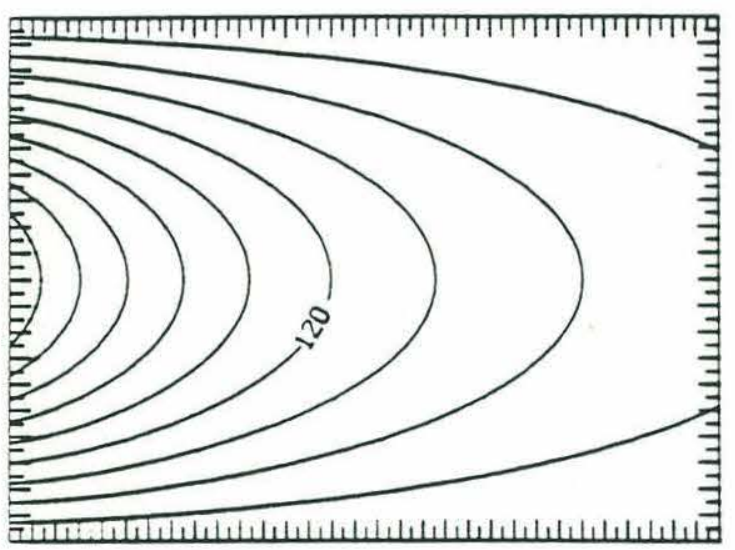

(b)

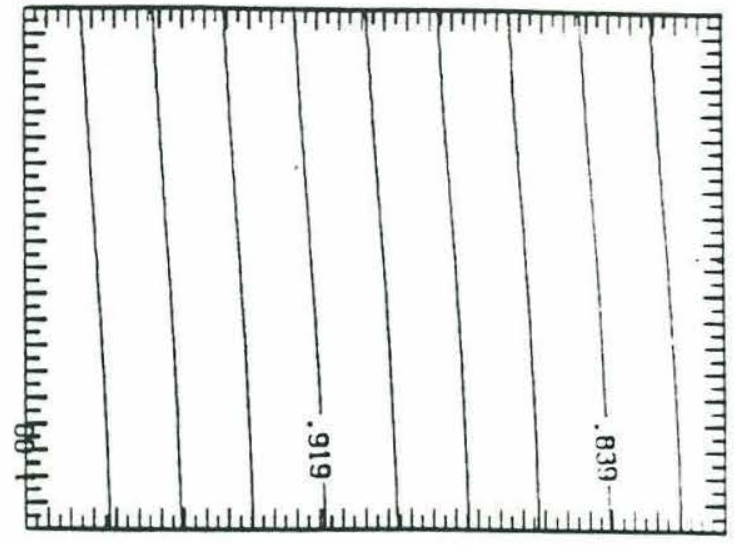

(c)

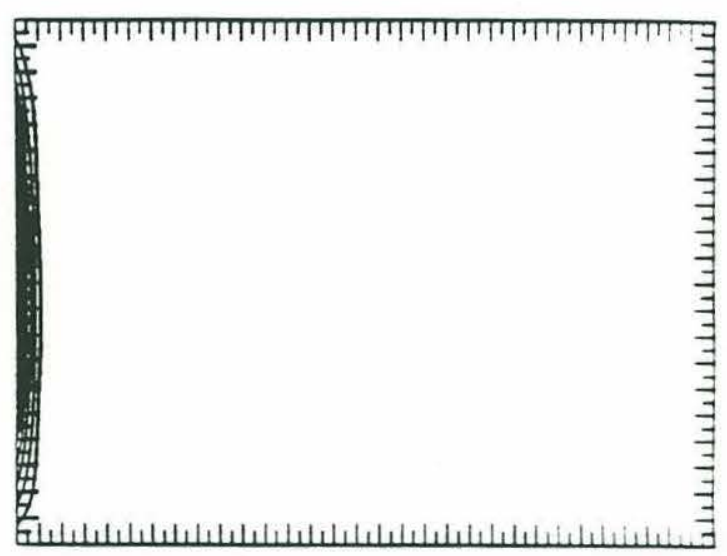

(d)

Figure 2.1: Contours of $b((\mathrm{a})$ and (c)) and $\psi((\mathrm{b})$ and (d)) for a one-dimensional fluid column at a constant rate of buoyancy loss. The horizontal and vertical axes are time and depth, respectively. The plots are for nonoverturning case ((a) and (b)) and overturning case ((c) and (d)). 


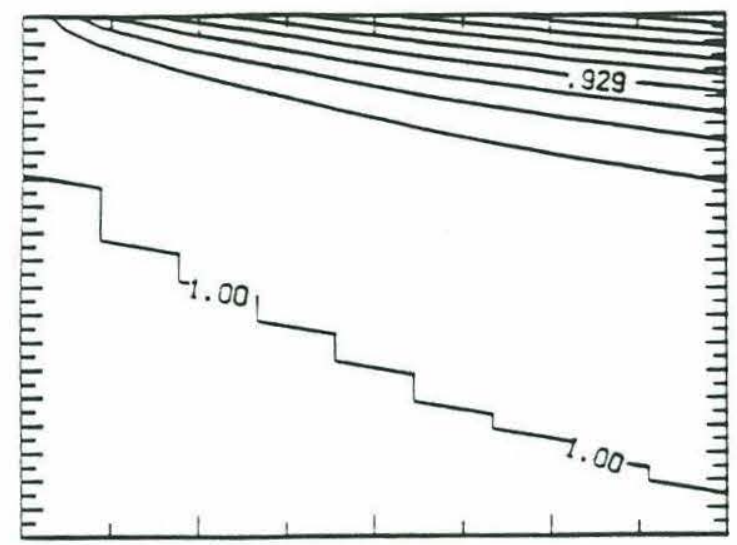

(a)

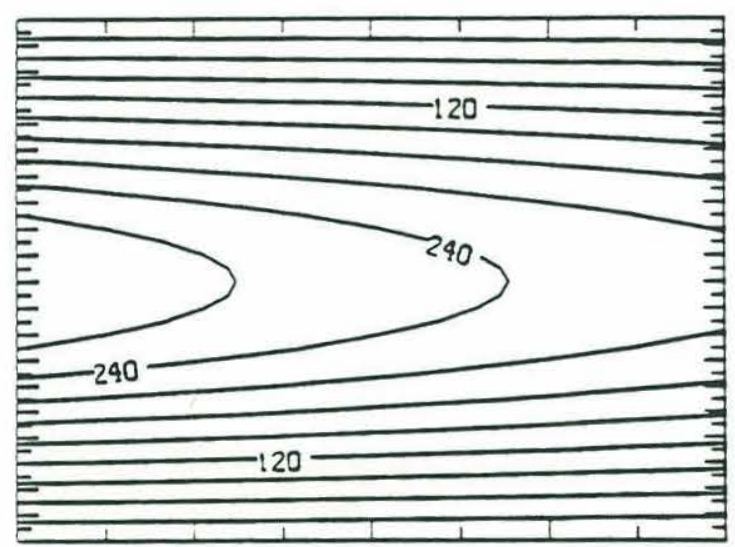

(b)

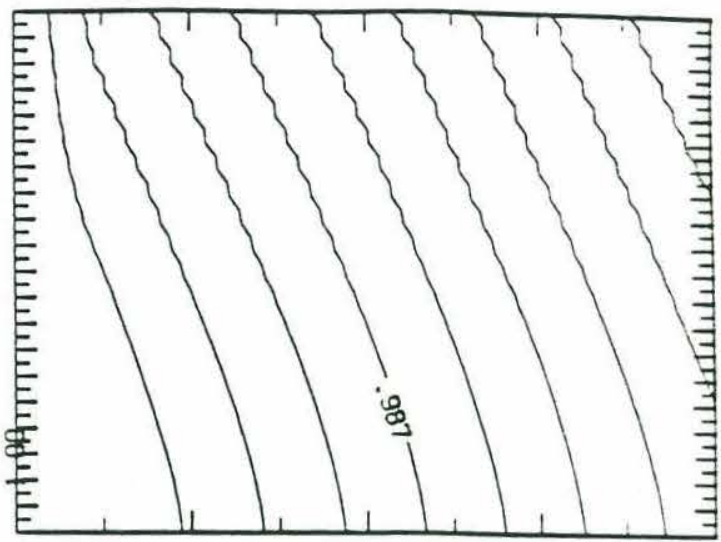

(c)

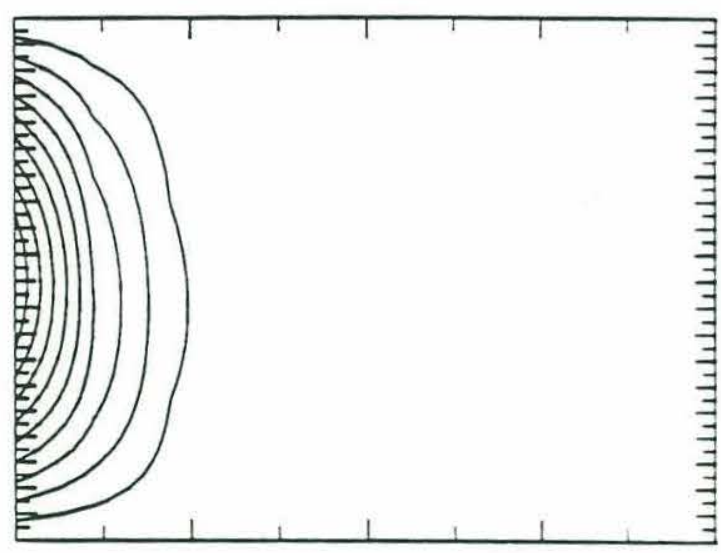

(d)

Figure 2.2: An enlarged view of figure 2.1 with only the first $1 / 6$ of the total time steps. 


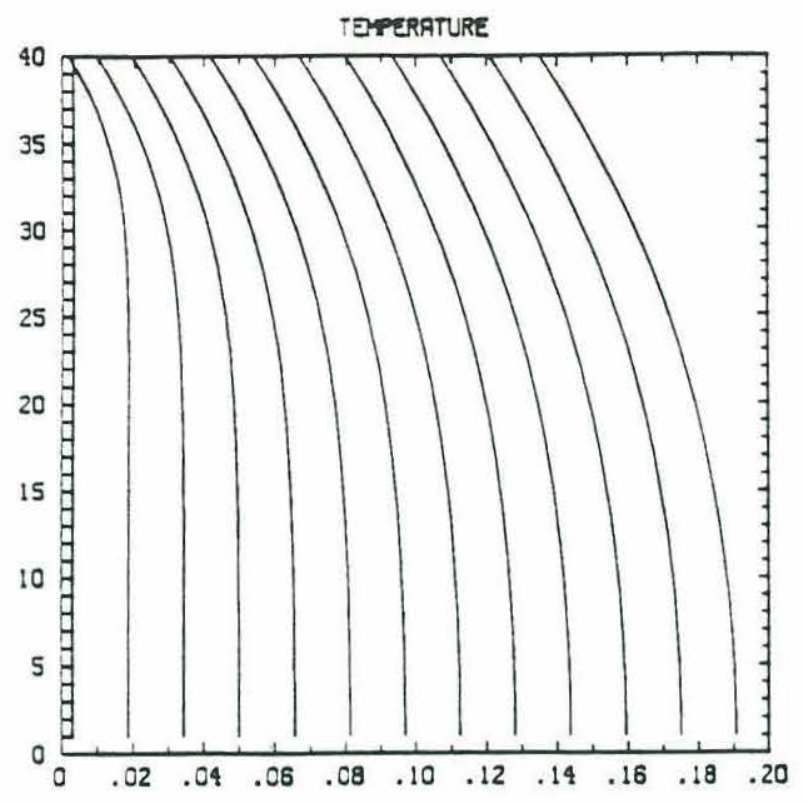

(a)

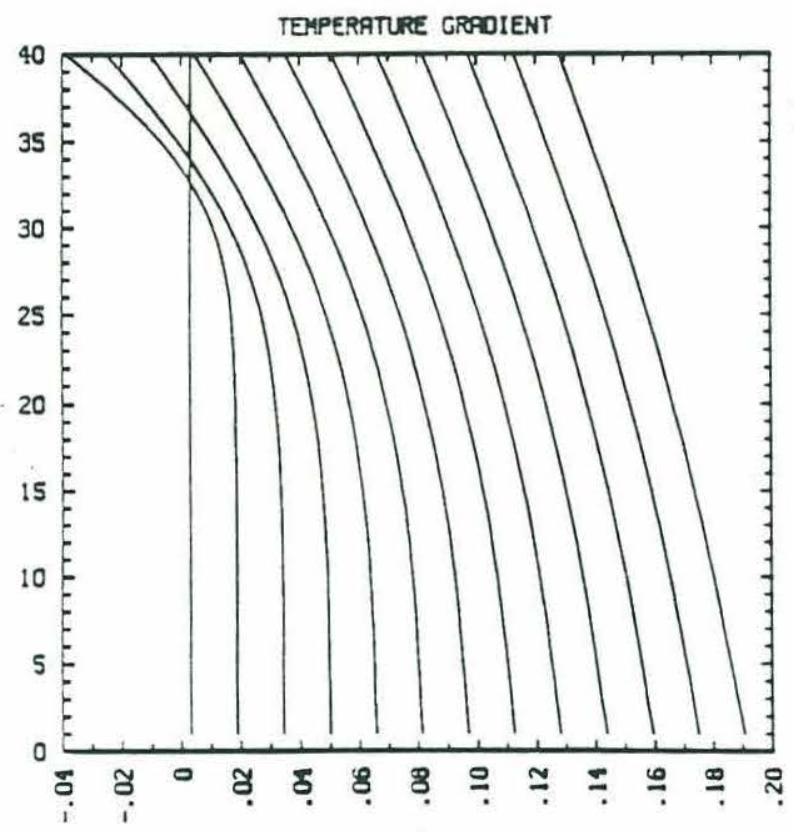

(b)

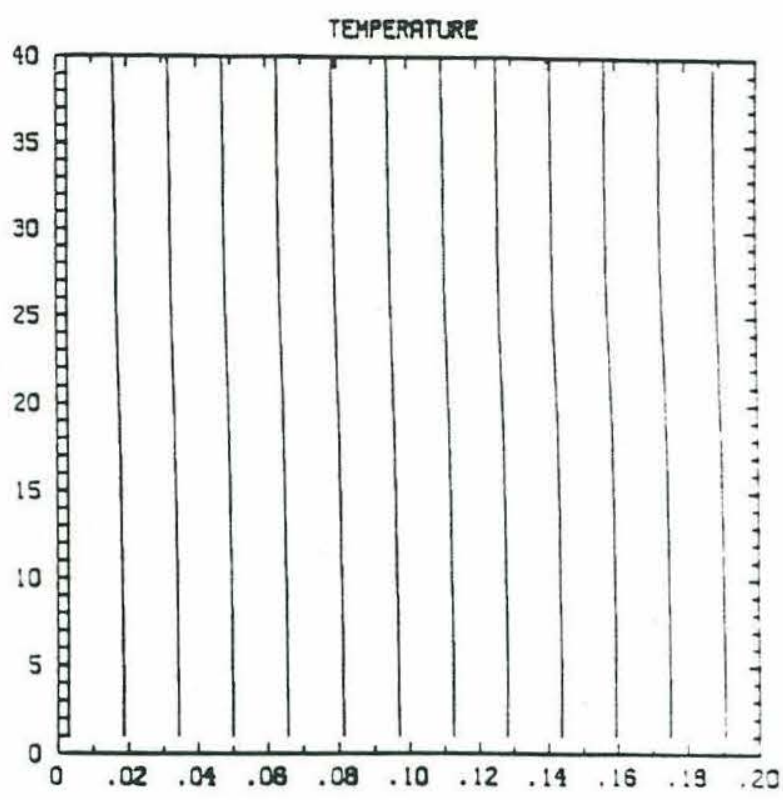

(c)

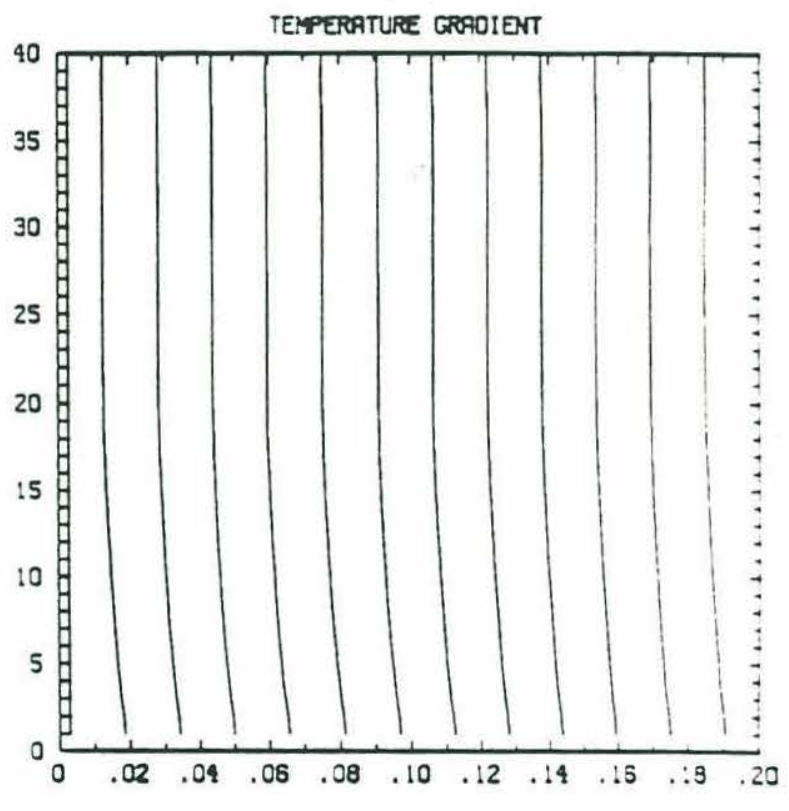

(d)

Figure 2.3: $b((\mathrm{a})$ and $(\mathrm{c}))$ and $b_{\boldsymbol{z}}((\mathrm{b})$ and $(\mathrm{d}))$ as functions of depth (vertical axis) at thirteen different time (horizontal axis). There is a constant rate of buoyancy loss at surface. The plots are for nonoverturning case ((a) and (b)) and overturning case ((c) and (d)). 

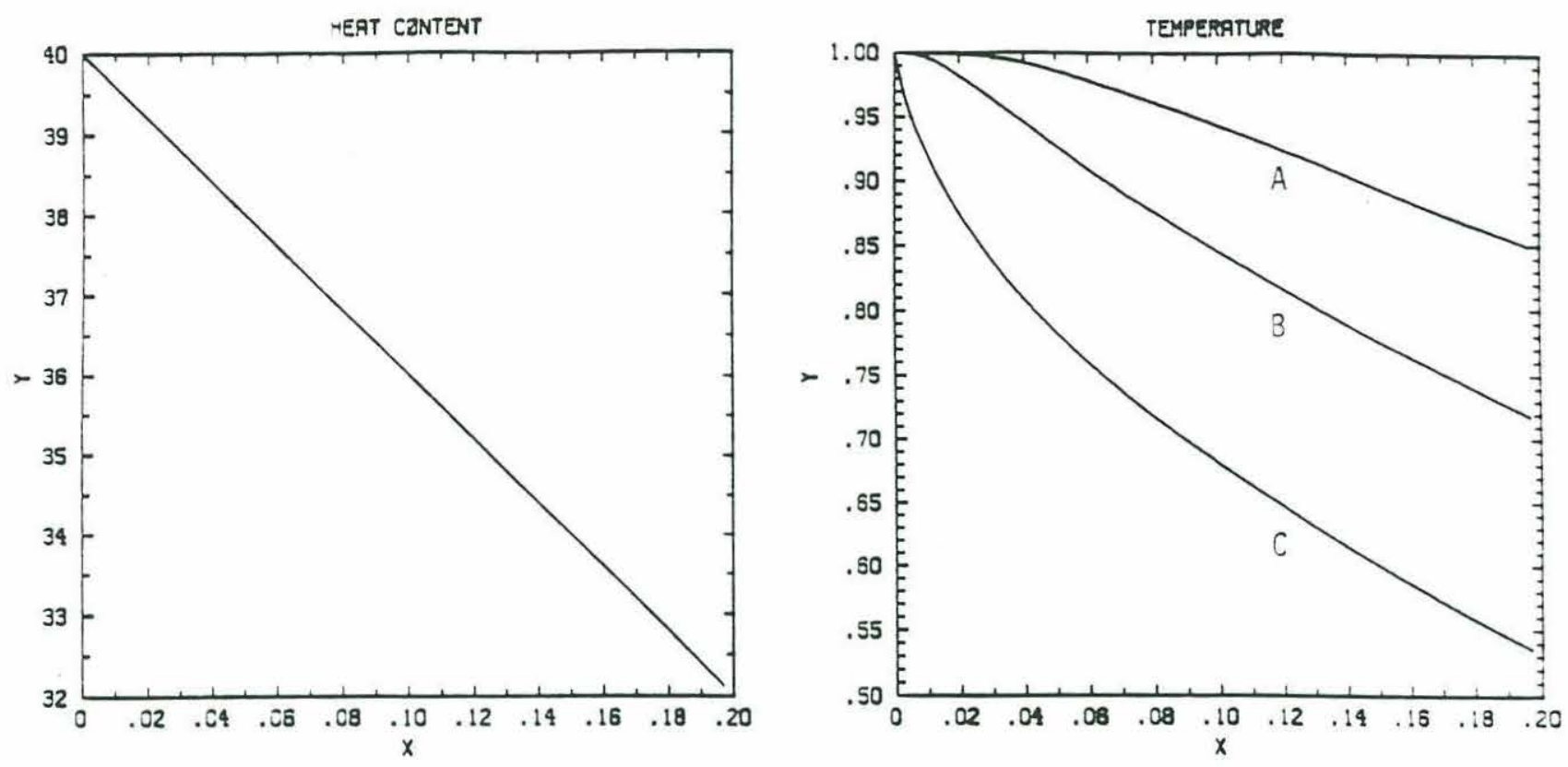

(a)

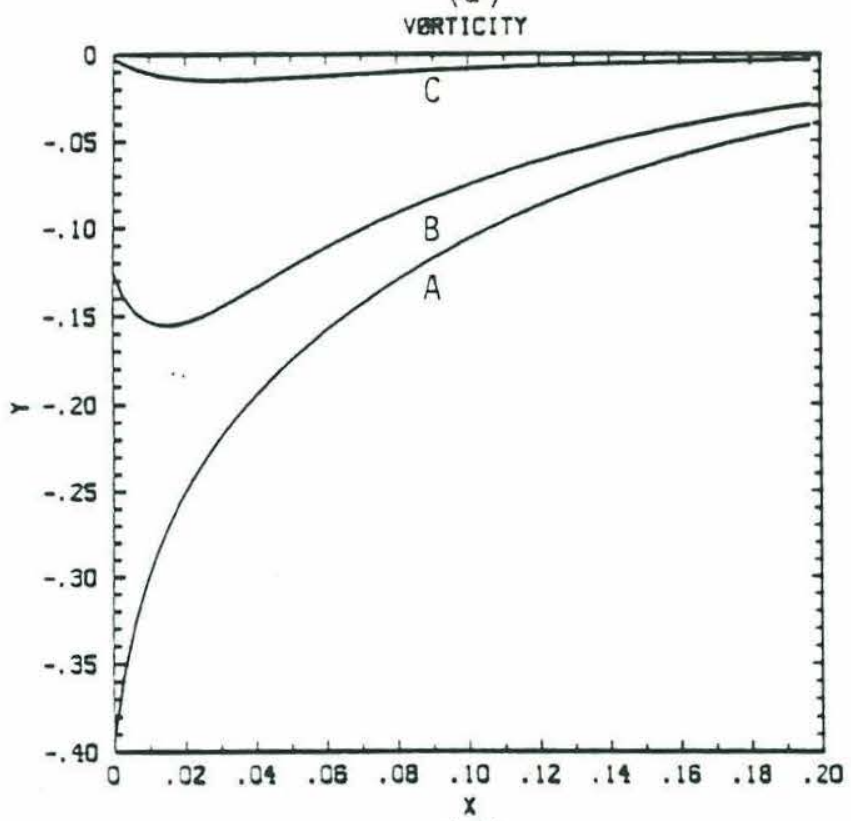

(c)

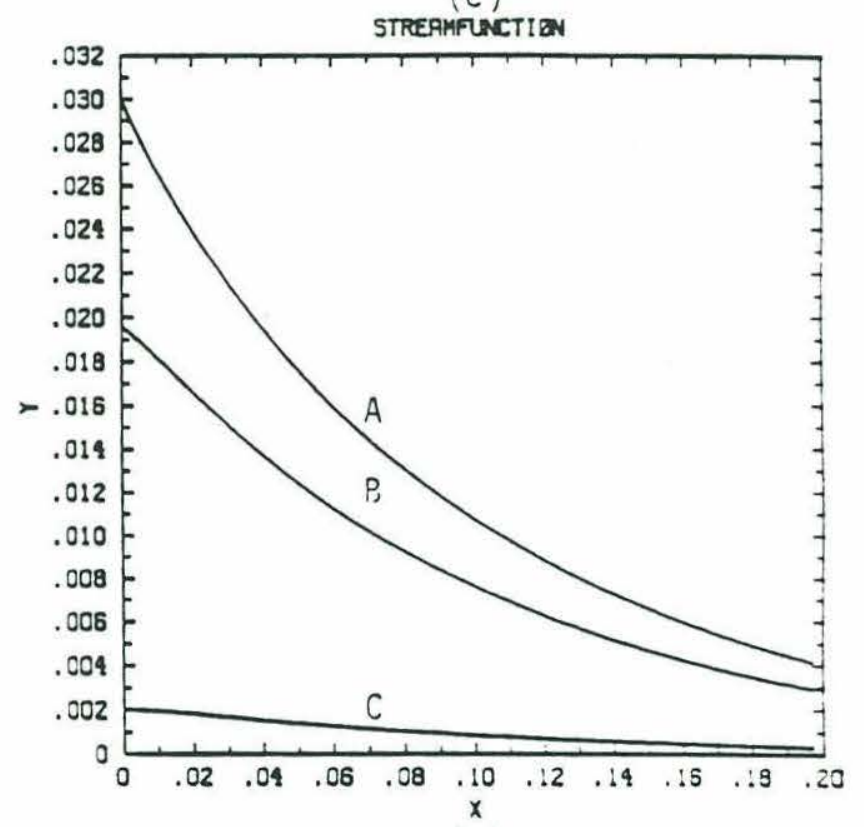

(d)

Figure 2.4: (a) the total heat content of the fluid column (vertical axis) as a function of time (horizontal axis). Values of $\psi_{z z}$ (b), $b$ (c) and $\psi$ (d) as functions of time at three different depth: (A) $1 / 2$, (B) $1 / 4$ and (C) $1 / 40$. The depth is normalized between 0 at surface and 1 at bottom. No overturning mixing is involved. 


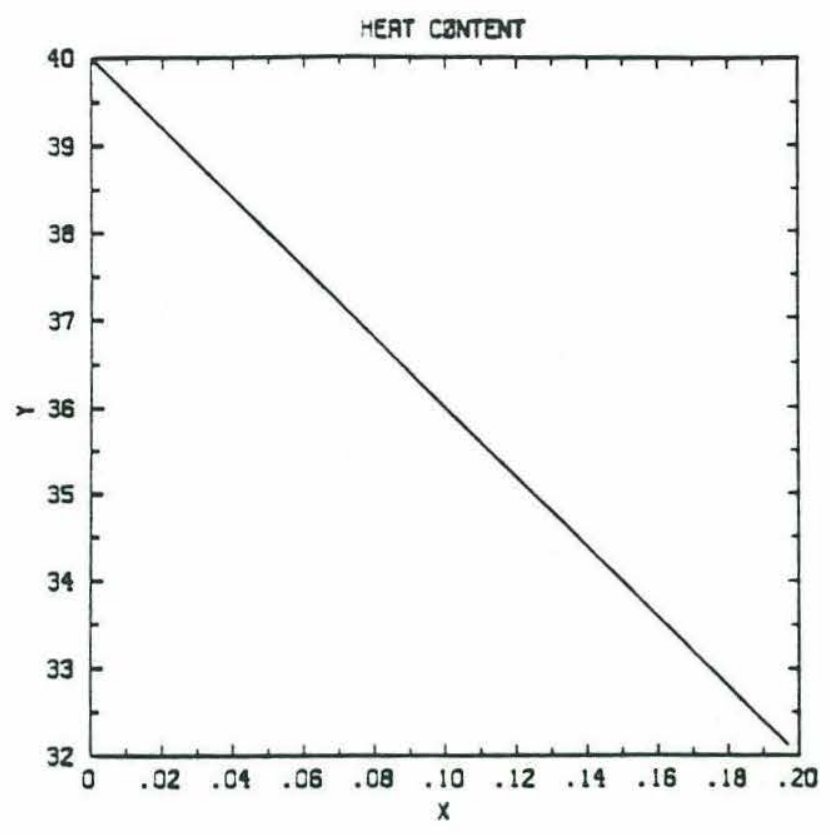

(a)

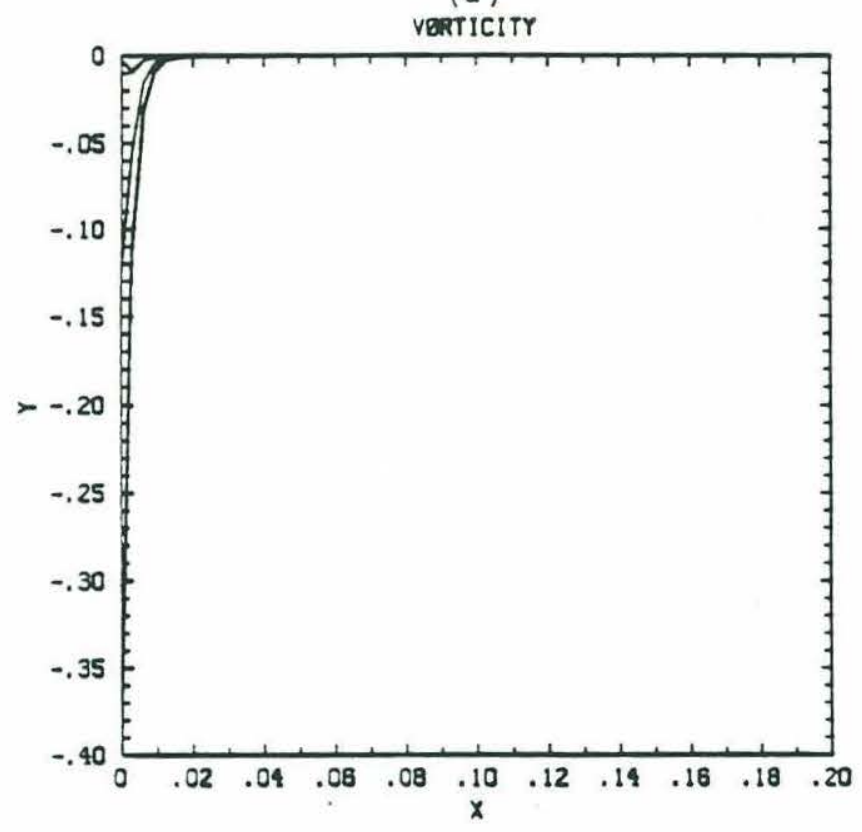

(b)

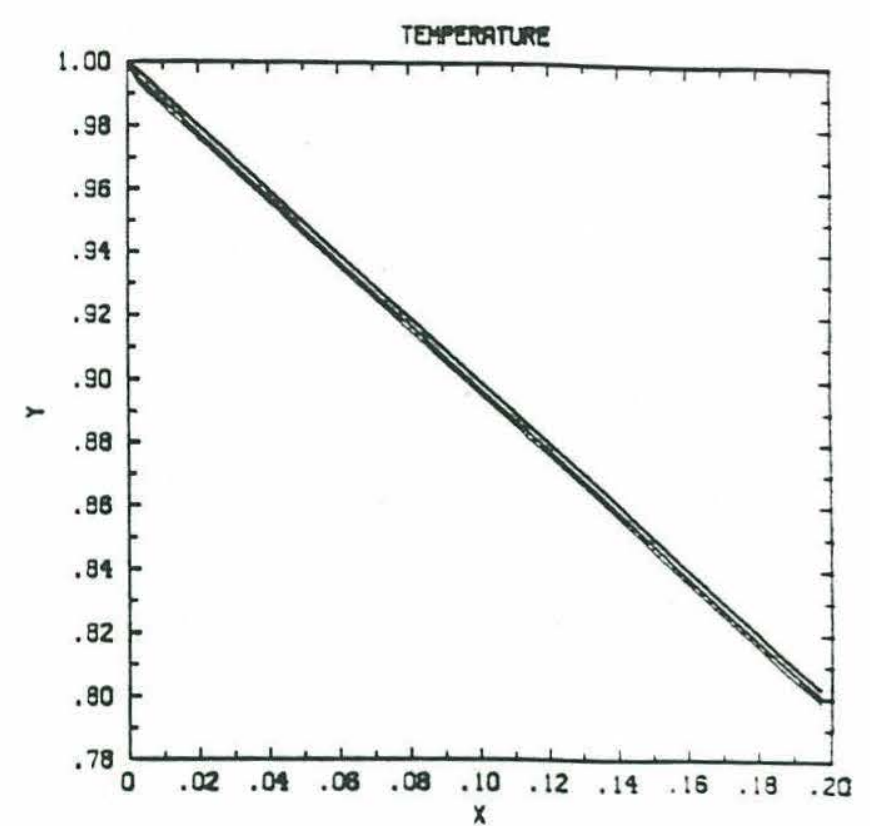

(c)

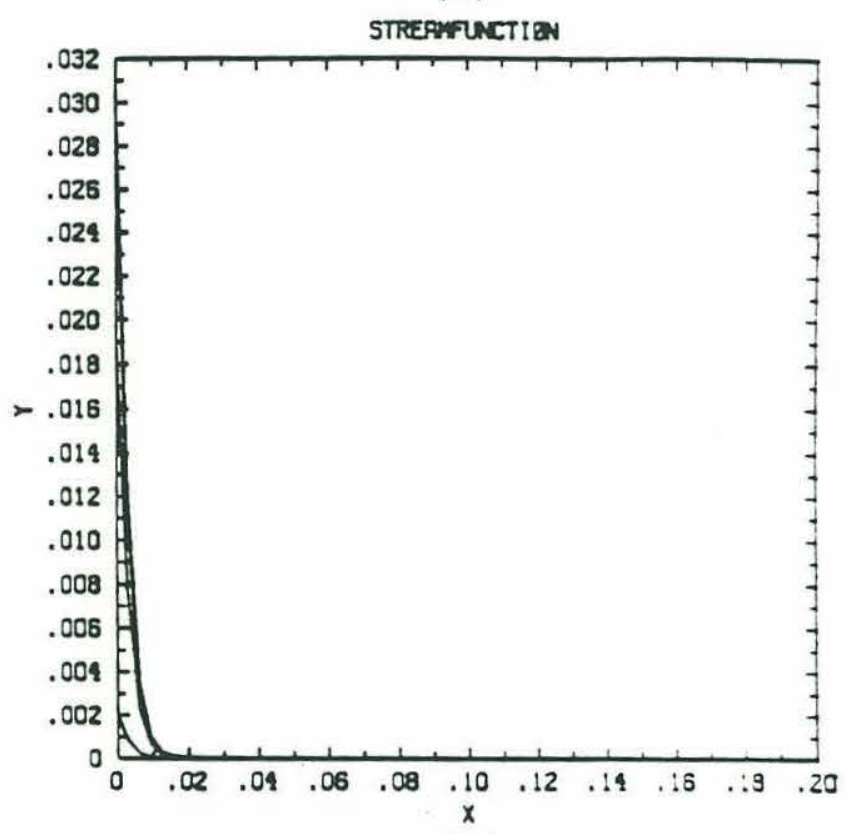

(d)

Figure 2.5: The same as figure 2.4, but for overturning case. 


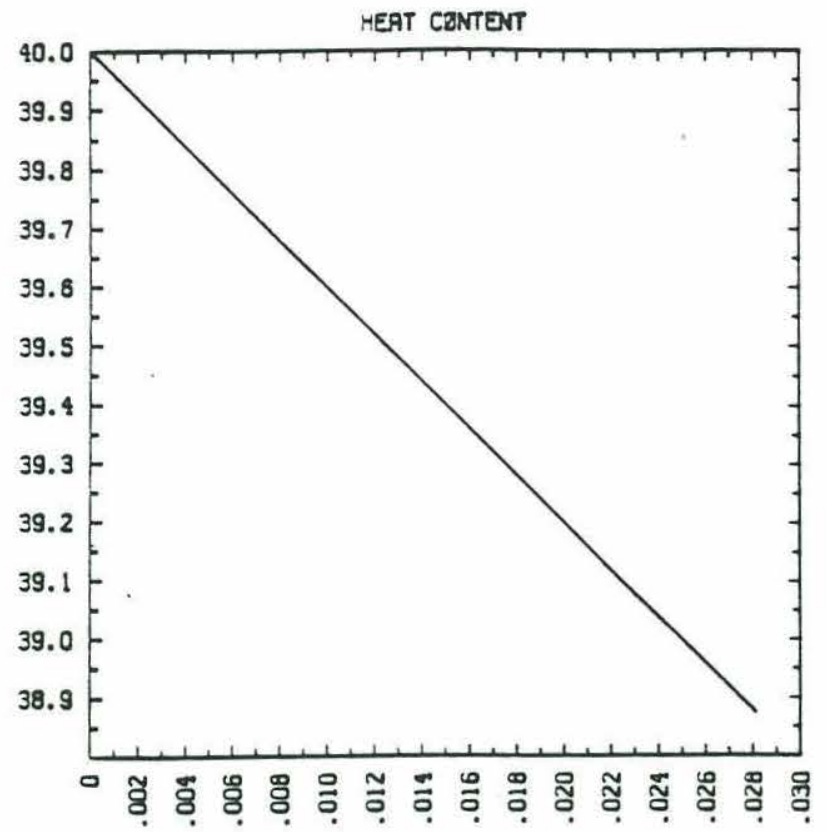

(a)

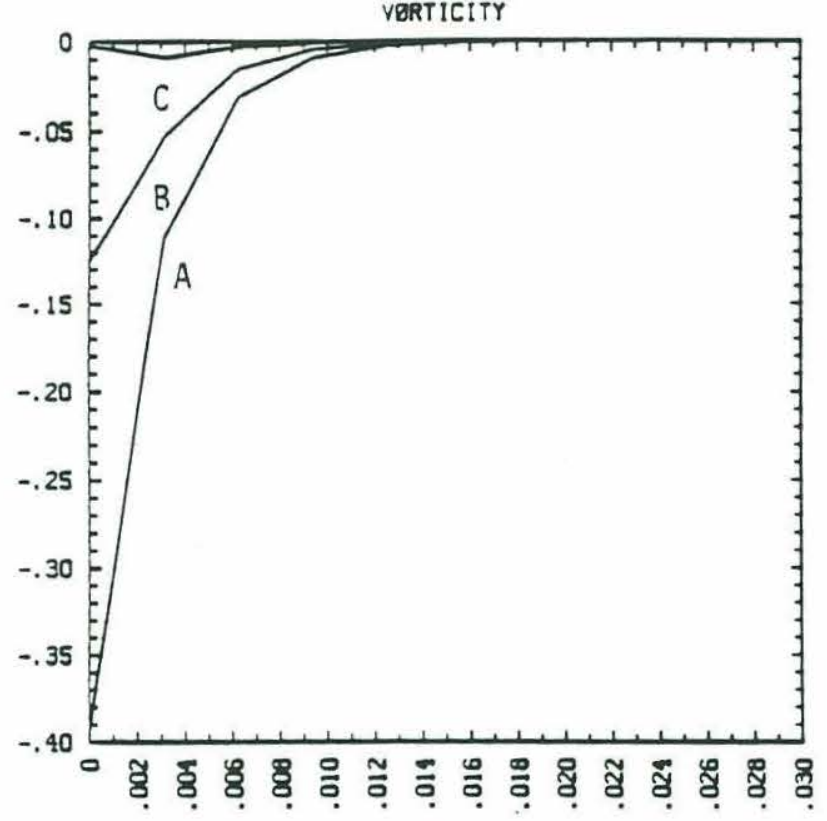

(b)

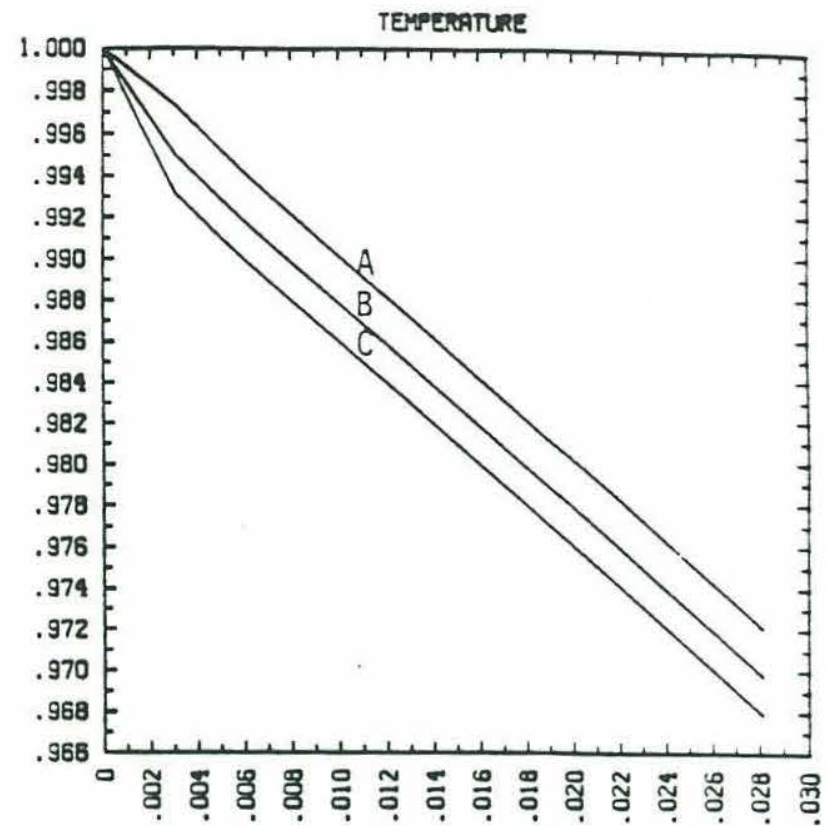

(c)

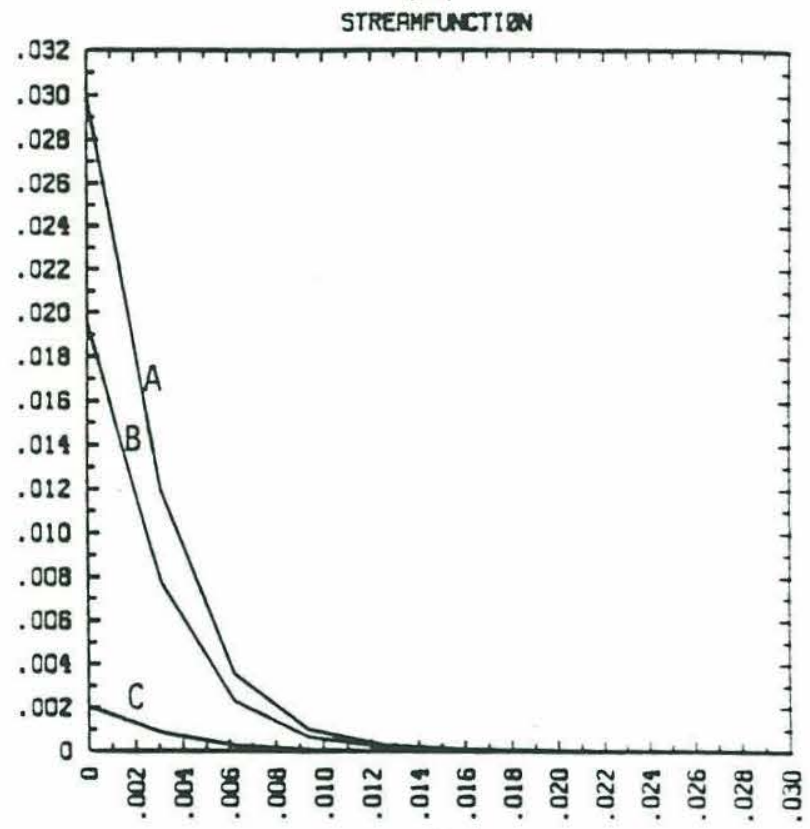

(d)

Figure 2.6: An enlarged view of figure 2.5 with only the first $1 / 6$ of the total time steps. 


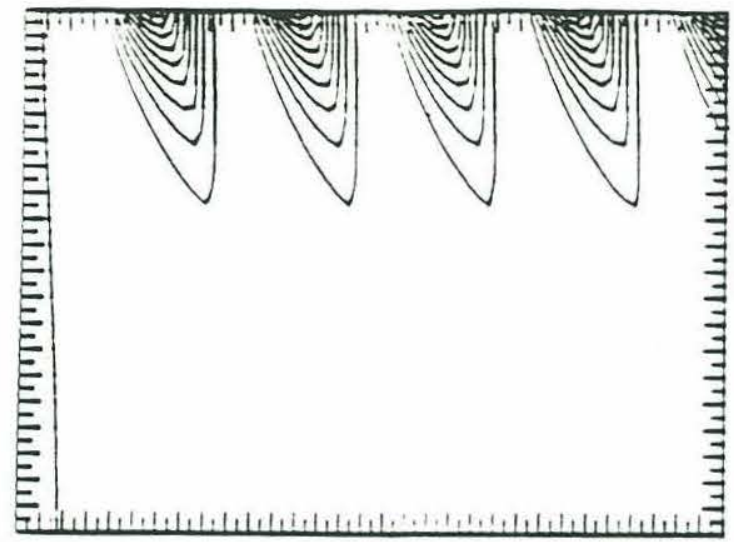

(a)

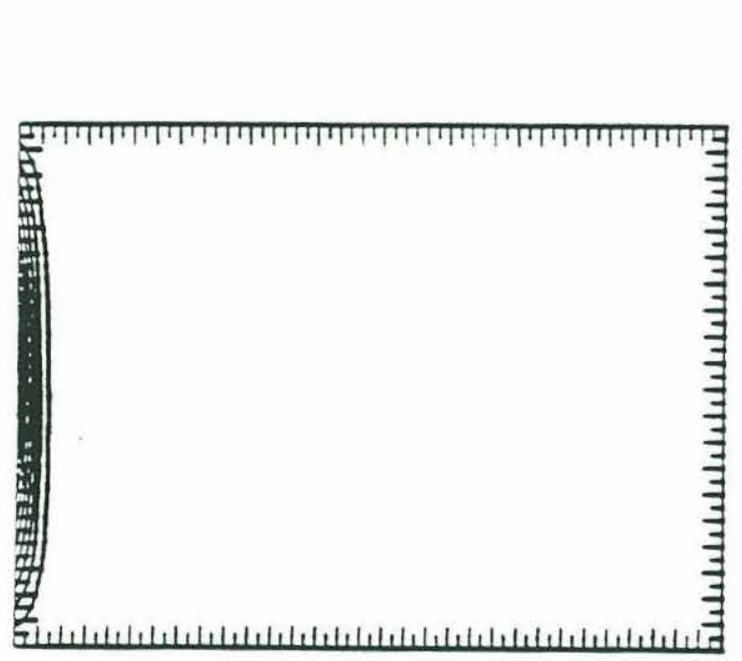

(b)

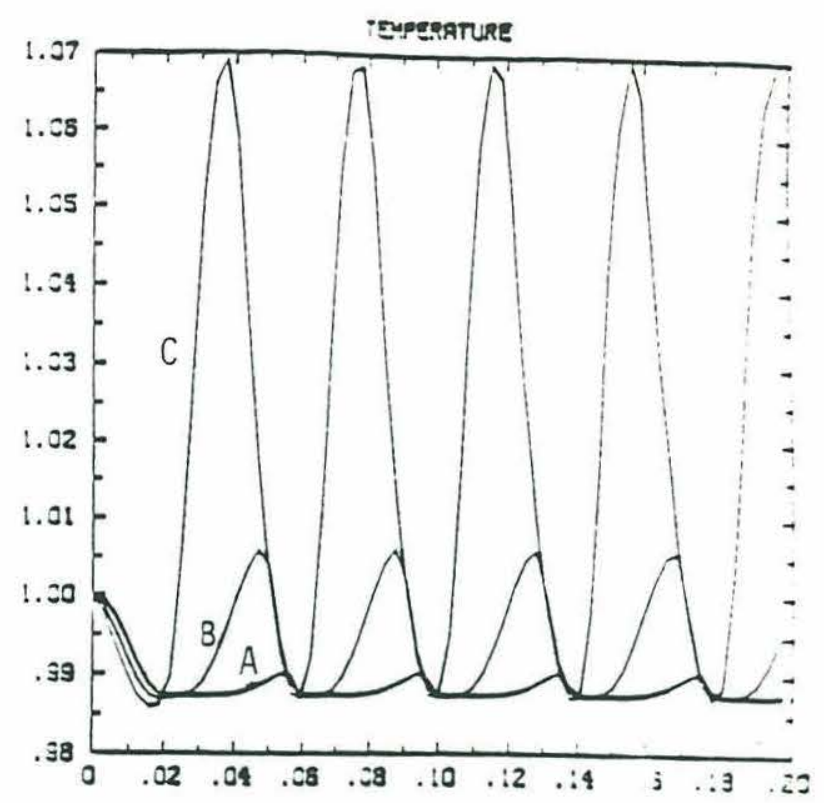

(c)

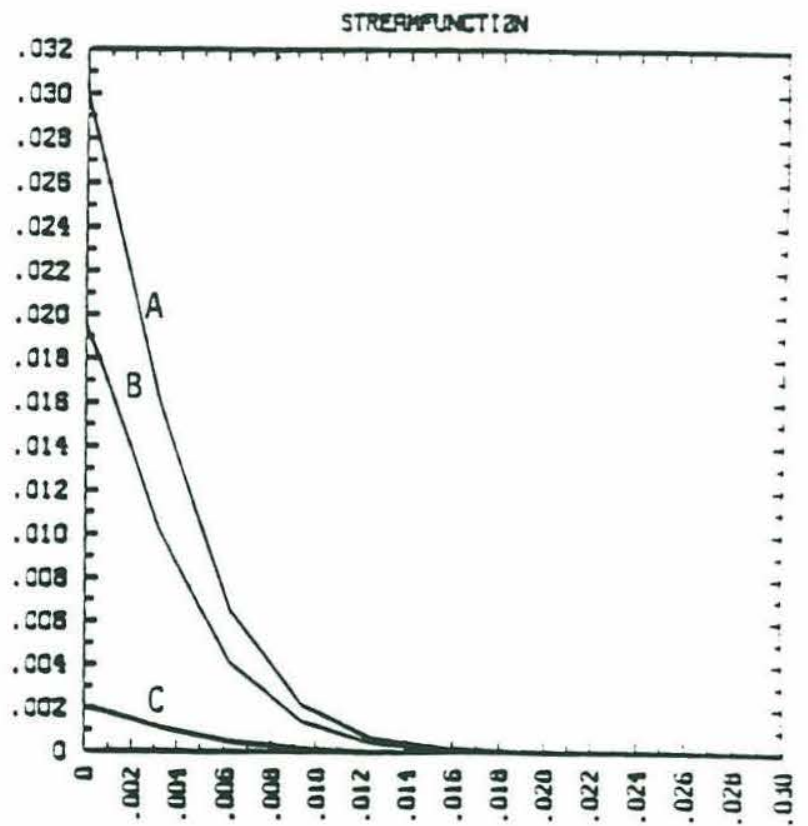

(d)

Figure 2.7: Contours of $b(\mathrm{a})$ and $\psi(\mathrm{b})$ for a one-dimensional fluid column at a periodic surface buoyancy flux. The horizontal and vertical axes are time and depth, respectively. (c) and (d) are $b$ and $\psi$ vs. time at three different depth: (A) $1 / 2$, (B) $1 / 4$, (C) $1 / 40$, respectively. Overturning mixing is used. 


\section{Chapter 3}

\section{Mixing and Circulation in a Two-Dimensional Basin Driven by Surface Buoyancy Flux}

\subsection{Introduction}

One of the most important mechanisms missed in the one-dimensional system examined in chapter two is the coupling between the thermal and the momentum fields. In the real fluid, it is known that a horizontal nonuniformity of temperature field will induce thermal wind in a rotating framework or generate motion with a horizontal component of vorticity in a nonrotating framework. In both situations, thermal energy is converted into kinetic energy, maintaining fluid motion in the presence of viscous dissipation. This thermally induced circulation is sometimes called the thermohaline circulation.

In many aspects, the problem of the oceanic thermohaline circulation is theoretically complicated and difficult to solve. The difficulties, to name a few, include the turbulent nature of mixing, the extremely wide spectra of the mixing scales and eddy diffusivities, and the role of nonlinearities in the circulation. For practical reasons, direct observations of thermohaline circulation are also not available to quantify its structure in detail. However we do in general have a qualitative picture, as already described in the introduction to chapter one. It is obvious that at this stage some further qualitative understanding of the problem will be helpful in many ways. Naturally 
we will look for a simplified system which contains the essence of the thermohaline circulation and illustrates the basic mechanisms.

Examples have been given by Stommel (1950 and 1962), as he elegantly demonstrated the inherent asymmetric nature of the thermohaline circulation and the smallness of sinking areas in the ocean by means of simple models. That idea has thus been followed and the work has been carried on by a number of researchers. Those works, (some of them were reviewed in chapter one) all reproduced Stommel's qualitative results and have retained the two-dimensional framework and a rectangular basin to avoid unnecessary complication. The system that will be used in this study is essentially the same as most of the previous works, with the usual advection-diffusion equations for both buoyancy and vorticity and an idealized basin geometry. In addition we implement the turbulent mixing parameterization for both buoyancy and momentum equations presented in the previous chapter. This study is still in the spirit of Stommel's pioneering work and is a continuation of the work of Beardsley and Festa (1972) and Nelken (1987).

In this chapter we shall examine the idealized thermohaline circulation in a horizontally stretched basin with arbitrarily small aspect ratio $(\lambda \leq 1)$, which bears more resemblance to the geometry of the ocean. Then we shall examine the Rayleigh number dependence of the system, a much studied problem in the past. The cases range from the diffusive limit at small $R_{a} \lambda^{2}$ values to the advective-diffusive limit at large $R a \lambda^{2}$ values. We shall raise the Rayleigh number to an unprecedented high value, $2 \times 10^{12}$, corresponding to $R a \lambda^{2}=2 \times 10^{6}$. Then the parameterization developed and tested in the chapter two is applied to examine the effect of turbulent mixing of density and momentum on a steady state thermohaline circulation. Finally the characteristic structure of the circulation and the thermal field will be studied using a scale analysis to investigate the dominant balance in the boundary layers. 


\subsection{The Model}

We will study here the problem of mixing and circulation driven purely by thermal forcing in the absence of rotation. The fluid is assumed to be incompressible and Boussinesq and confined to a channel which is infinitely long in the $x$-direction and rectangular in $y-z$ cross-section. Under these assumptions equation (1.5) can be rewritten as follows:

$$
\begin{aligned}
\frac{1}{\sigma}\left[u_{t}+J(\psi, u)\right] & =\bar{\nu}_{H} u_{y y}+\left(\nu_{V} u_{z}\right)_{z} \\
\frac{1}{\sigma}\left[\left(\nabla^{2} \psi\right)_{t}+J\left(\psi, \nabla^{2} \psi\right)\right] & =R_{a} \lambda^{2} b_{y}+\bar{\nu}_{H}\left(\nabla^{2} \psi\right)_{y y}+\frac{\partial}{\partial z}\left[\nabla \cdot\left(\nu_{V} \nabla \psi_{z}\right)\right] \\
b_{t}+J(\psi, b) & =\bar{\kappa}_{H} b_{y y}+\left(\kappa_{V} b_{z}\right)_{z}
\end{aligned}
$$

where the definition of the symbols is given in chapter one. In these dimensionless equations buoyancy can be considered to be the same as a dimensionless temperature. In the discussion below we will sometimes describe the buoyancy (or density) in terms of temperature.

Because of the absence of rotation, the zonal velocity $u$ enters only in the $x$ momentum equation (3.1a). The thermal forcing has no effect on generating or maintaining the zonal momentum field. If initially the zonal flow field is zero and $u$ vanishes on all boundaries, it is zero forever. Since our focus is on the mixing and circulation in the meridional cross-section, equation (3.1a) can be eliminated from the system.

The Prandtl number $\sigma$, characterizing the ratio of the viscous to diffusive effects on the fluid, is usually one or larger for the ocean. The study of Beardsley and Festa (1972) showed that when $\sigma$ varies from 1 to 100 , the corresponding variance of the maximum value of streamfunction is less than $1 \%$. Considering their and other relevant studies (Somerville, 1967), Beardsley and Festa suggested that for a certain range in $R_{a}-\sigma$ space, the solutions are virtually independent of Prandtl number. The value they used in their numerical simulation was constant, $\sigma=10$. Since this value is based only 
on the molecular viscosity and diffusivity for water, a property of the fluid, it does not involve any adjustment caused by turbulent effects. In this study, we will deal with steady, laminar motions driven purely by buoyancy forcing at a scale comparable to the ocean, so the value of $\sigma$ is assumed to be one. Even though later we will parameterize turbulent mixing, changing $\kappa_{V}$ and $\nu_{V}$ to large values in statically unstable regions, the value of $\sigma$ will remain unity. Since the results differ by less than $1 \%$ within a certain $\sigma$ range (Beardsley and Festa, 1972) we should be able to reproduce most of the previous results.

It is important to note that if there were no meridional temperature gradient, there would be no thermally driven circulation. Thus in equation ( $3.1 \mathrm{~b})$ the first term on the right hand side, $R_{a} \lambda^{2} b_{y}$ is vital to the existence of a vertical circulation field. In the ocean $R_{a}$ is about $10^{17}$ and $\lambda$ about $10^{-3}$, yielding $R_{a} \lambda^{2}=10^{11}$. This means that even a very small $b_{y}$ may result in a $O(1)$ basinwide meridional circulation.

In chapter one we defined the Rayleigh number $R_{a}=B D^{3} / \nu \kappa$, where we took $B$ to be a scale quantity for buoyancy. Since we will specify the surface buoyancy flux, $Q_{b}=Q / \kappa_{V}=\partial b / \partial z$, it is convenient and physically consistent to define a Rayleigh number in terms of the magnitude of this flux. Replacing $B$ by the surface flux $Q_{b} D$ in Rayleigh number gives $R_{a}=Q_{b} D^{4} / \nu \kappa$. Since $\nu$ and $\kappa$ are vertical components of eddy viscosity and diffusivity, this $R_{a}$ is also regarded as 'vertical' Rayleigh number with respect to the conventional 'horizontal' Rayleigh number defined with horizontal components and temperature difference. This horizontal Rayleigh number is most often used when a boundary temperature is specified instead of a flux, such as studies by Somerville (1967) and Beardsley and Festa (1972). In the present study the focus is on vertical mixing and circulation for a given boundary flux; thus it is advantageous to use the 'vertical' Rayleigh number. Thus, to change the Rayleigh number by a factor, we simply multiply the boundary flux by the same factor and leave the other model parameters unchanged. 
Most of the previous studies used an aspect ratio equal or close to unity (Table 3.1). The exceptions are the studies of Stommel (1950) and Somerville (1967), who examined the cases of small aspect ratio. Stommel (1950) required $R_{a} \lambda^{2} \ll 40$, Somerville (1967) used a fixed $\lambda=0.1$. For the large scale oceanic circulations, as we discussed above, $R_{a} \lambda^{2}$ is as high as $10^{11}$ and $\lambda$ is $10^{-3}$. Thus, Stommel's value is obviously too small, and Somerville's value is far from the oceanic situation. In this thesis we will try to fill this gap by studying cases with aspect ratios ranging from 1 to 0.001 and $R_{a} \lambda^{2}$ as large as $2 \times 10^{6}$. However, due to limited computer resources we still can not reach the realistic value of $R_{a} \lambda^{2}$, because of the enormous resolution requried at high $R_{a}$ values. A list of parameters used in this and previous studies is given in Table (3.1).

The boundary conditions required to solve this system are no flux of buoyancy through side walls and bottom boundary (they are perfectly insulated), and no stress and no flow across all boundaries; at the top boundary the buoyancy flux is specified.

$$
\begin{aligned}
\bar{\kappa}_{H} \frac{\partial b}{\partial y} & =0, \quad \text { at } y=0,1 \\
\kappa_{V} \frac{\partial b}{\partial z} & =0, \quad \text { at } z=-1 \\
\kappa_{V} \frac{\partial b}{\partial z} & =Q, \quad \text { at } z=0 \\
\psi=\nabla^{2} \psi & =0, \quad \text { at } y=0,1 \text { and } z=0,-1
\end{aligned}
$$

The last condition for zero vorticity comes directly from the no tangential stress condition, which is also called the free slip boundary condition. The boundary conditions used in this and other studies are listed in Table (3.1). Some previous investigators used rigid boundary conditions, i.e., both normal and tangential velocities vanish on the boundaries (Beardsley and Festa, 1972). The rigid conditions are more suitable for describing laboratory results, while the free conditions give a simple numerical formulation. The choices of boundary conditions may result in differences in some of the details in the solution, such as changes in values of maximum streamfunction and shear near boundaries, especially in the narrow boundary layer region, where a stress condition 
brings the velocity to zero, and thus causes larger shear dissipation; but it should not cause any fundamental difference in the pattern of the solution. In this study we have used free slip boundary conditions for all cases.

The surface flux of buoyancy has a cosinusoidal distribution along meridional direction, $Q=\cos (\pi y)$, so that the net flux into the system is zero at all times. Fixed temperature boundary conditions were used by previous investigators (Somerville, 1967; Beardsley and Festa, 1972). In this case, a linear or cosinusoidal temperature distribution was given along the bottom boundary. Their results showed a slight increase of basin average temperature with Rayleigh number. This is avoided in the present study because there is no net flux into the system. Even though both conditions lead to a steady state solution, we prefer the flux condition to the temperature condition. For the flux condition chosen here, the heat is always gained at the southern half of the basin and lost at the northern half. The balance of the flux in the domain requires a net heat transport across the basin centerline $y=1 / 2$. This is achieved through the diffusion of heat and more effectively through advection. This fluid motion, with a cellular pattern, is driven by the horizontally inhomogeneous buoyancy distribution.

\subsection{Numerical Scheme}

Since the system described in section (3.2) is highly nonlinear, it will be solved numerically. The differential equations in (3.1) are rewritten in their finite difference forms and are solved on a space-staggered Arakawa C-grid system (Arakawa and Lamb, 1977). The advection of buoyancy and vorticity are approximated using the Arakawa Jacobian (Arakawa, 1966), which conserves temperature and temperature squared, and vorticity and kinetic energy. The horizontal and vertical diffusion of buoyancy and horizontal diffusion of vorticity are approximated by a second-order central-difference 
scheme. The vertical diffusion of vorticity is slightly complicated and is written as

$$
\frac{\partial}{\partial z}\left[\nabla \cdot\left(\nu_{V} \nabla \psi_{z}\right)\right]=\frac{\partial^{2}}{\partial z^{2}}\left(\nu_{V} \nabla^{2} \psi\right)-\lambda^{2} \frac{\partial}{\partial z}\left(\nu_{V z} \psi_{y y}\right)
$$

to avoid direct calculation of a fourth-order derivative in $z$. The right hand side terms of the above equation are then approximated using a second-order central difference scheme.

The difference equations are stepped forward in time. The independent variables are discretized uniformly, $y_{i}=i \Delta y$ and $z_{j}=j \Delta z$, for $i=0,1, \ldots, I$ and $j=0,1, \ldots, J$, $t_{n}=n \Delta t$, for $n=0,1,2, \ldots$, where $\Delta y$ and $\Delta z$ need not be the same, and $\Delta t$ has to satisfy the Von Neumann stability criterion,

$$
\Delta t \leq \frac{1}{2\left(\frac{\kappa_{H}}{\Delta y^{2}}+\frac{\kappa_{V}}{\Delta x^{2}}\right)+\frac{\psi_{m a s}}{\Delta y \Delta z}} .
$$

If there exists a stable solution for this system under a time invariant boundary condition, the final steady solution has to be independent of the initial condition and only depends on the boundary condition given. This means that one can start the calculation with any initial condition and the solution may differ only by a constant. For simplicity we use zero momentum and constant buoyancy as the initial conditions.

The numerical calculation proceeds as follows: first the new buoyancy field is computed by integrating the buoyancy equation forward in time under the given boundary flux condition; then the vertical component of diffusion and dissipation coefficients are calculated as functions of vertical buoyancy gradient. The vorticity equation is then stepped forward in time using the new buoyancy field and $\nu_{V}$ from the above calculation, and finally the streamfunction is obtained using a Poisson solver (Buneman, 1969). Using the new values of the streamfunction, vorticity and $\kappa_{V}$, we proceed to next time step for the buoyancy field. The leapfrog stepping scheme is used (Press etc.,1986). The loop is repeated until a steady state for both the buoyancy and momentum fields is achieved. Here the steady state is defined by relative changes of both 
the buoyancy and momentum fields between two adjacent time steps being less than $0.01 \%$ at every grid point.

The requirement for resolution is variable for different values of Rayleigh number and aspect ratio. As mentioned in section (3.2), we must lower the Rayleigh number in order to get a reasonable grid resolution, since at large Rayleigh number the circulation becomes excessively asymmetric, and a very narrow boundary layer develops along one of the vertical walls. To resolve this boundary layer a large number of grid points is needed. One possible remedy for this problem is to use uneven grid spacing with a high grid density in the boundary layer. This would complicate the numerical formulation and has not been used here. In this thesis the values of $R_{a} \lambda^{2}$ ranges from $2 \times 10^{4}$ to $2 \times 10^{6}$, and the corresponding appropriate grid resolution ranges from $17 \times 17$ at the lowest value to $65 \times 33$ at the highest value of $R_{a} \lambda^{2}$.

All numerical computations were performed on a Sun microsystem 4/260 computer. The CPU time used for one simulation ranges from five minutes for low $R_{a} \lambda^{2}$ with $17 \times 17$ grids to thirty hours for high $R_{a} \lambda^{2}$ with $65 \times 33$ grids.

\subsection{On the Aspect Ratio of the Basin}

One of the principal purposes of the previous and present studies is to examine the asymmetric circulation structure and to explain the extreme smallness of the sinking regions in the world oceans (Stommel, 1962). The notable shape of the ocean as a thin vertical layer of fluid with large horizontal extention has not been considered in previous investigations (see table 3.1). By choosing a vertical to horizontal aspect ratio close or equal to one, the solution will not be applicable to the thermohaline circulation. However all previous investigators were able to reproduce the fundamental features the asymmetry of the temperature and momentum fields, discovered by Stommel in his 1950 paper. It is still unclear how this asymmetry depends on the basin aspect 
ratio, how variations of the aspect ratio affect the balance of the dynamics and the thermodynamics of the system, and how the intensity of the circulation changes with the aspect ratio.

Those who used a small aspect ratio in their studies (Stommel, 1950; Somerville, 1967) also made important simplifications in their system. The smallness of the aspect ratio allowed them to neglect the horizontal diffusion of both heat and momentum. We shall see in section (3.7) that this is only correct outside the vertical boundary layer. As noted in Somerville (1967), his model is not capable of solving the asymmetry and the boundary layer structure accurately, especially at large Rayleigh numbers. He attributed these failures to the truncation of his Fourier expansion. Actually this is also due to the neglect of $T_{y y}$ and $\left(\nabla^{2} \psi\right)_{y y}$ in his equations. In the boundary layer the horizontal diffusion enters the leading order balance, and it can not be neglected totally.

Qualitatively the influence of the aspect ratio can be seen by examining equations (3.1b and c). The dimensionless horizontal diffusivity and viscosity are defined as $\bar{\kappa}_{H}=\lambda^{2} \kappa_{H} / \kappa$ and $\bar{\nu}_{H}=\lambda^{2} \nu_{H} / \nu$, where $\kappa_{H}$ and $\nu_{H}$ are the typical values of horizontal eddy coefficients and $\kappa$ and $\nu$ are the typical nonconvecting vertical ones. Woods (1973) showed that the values of $\kappa_{H}$ and $\nu_{H}$ change as the time and space scales of the flow field vary. Typical values for $\kappa_{H}$ and $\nu_{H}$ range from 50 to $5 \times 10^{4} \mathrm{~m}^{2} / \mathrm{s}$, and for $\kappa$ and $\nu$ from $10^{-5}$ to $10^{-2} \mathrm{~m}^{2} / \mathrm{s}$ in the open ocean (Killworth, 1976). For an aspect ratio 0.001, $\bar{\kappa}_{H}$ and $\bar{\nu}_{H}$ range from 0.5 to 5 . The scaling suggests that both $b_{y y}$ and $\left(\nabla^{2} \psi\right)_{y y}$ must be retained in the system. For convenience we simply let $\bar{\kappa}_{H}=\bar{\nu}_{H}=1$ in this study. For an aspect ratio close to the oceanic value, the horizontal diffusion is as important as the vertical one, and any attempt to neglect it must be justified. For other cases with larger aspect ratio the horizontal diffusion is at least as important as in the oceanic case. 
The fundamental influence of the aspect ratio from a mathematical point of view comes from the Poisson equation involving the stream function, $\nabla^{2} \psi=\lambda^{2} \psi_{y y}+$ $\psi_{z z}$. With $\lambda$ close to one, this is an elliptical equation, for which no real family of characteristic curves exist. For $\lambda \ll 1$, the equation degenerates to a second order ordinary differential equation in $z$ on $(y, z)$ plane, $\nabla^{2} \psi \approx \psi_{z z}$. Mathematically these two equations are quite different, but when solving for $\psi$, the double integration behaves like a smoothing operator in the space, so that contours of $\psi$ are considerablly smoother than those for $\nabla^{2} \psi$. The behavior of the system is unknown for the transition of the Poisson equation from PDE to ODE, but the physical constraints can only allow a gradual and smooth transition in momentum and temperature fields. Detailed analysis of the balance to the leading order will be given in section (3.7). In this section we shall examine some of the results from numerical solutions of the problem.

Figures (3.1) and (3.2) show the numerical results for steady state temperature (top panel), stream function (middle panel) and vertical temperature gradient (bottom panel) at five different values of aspect ratio and two different values of Rayleigh number. The thick line in the plots of the temperature and stream function divides the basin into two parts; the part to the left of the line has stable stratification, i.e., $b_{z}>0$, and the part to the right of the line has unstable stratification, i.e., $b_{z}<0$. $R_{a} \lambda^{2}$ is $2 \times 10^{4}$ in figure (3.1) and $2 \times 10^{5}$ in figure (3.2), and grid resolutions are $17 \times 17$ and $33 \times 17$, respectively. Looking at plots of $\lambda=1$, we see that the basic features shown by previous laboratory experiments and numerical simulations are reproduced, most importantly, the asymmetry of the temperature and stream function fields, and the uniform interior temperature in the lower half of the basin. The fluid is heated at the southern surface and advected to the north by circulation, where it is cooled and turns into a downward jet to form a northern boundary layer. This boundary current diverges and joins the interior fluid at depth. In the interior the cold fluid, approximately uniform in density, upwells slowly against a downward diffusion in the thermocline. 
As the aspect ratio decreases, i.e., the basin becomes flatter, the circulation gyre becomes more and more asymmetric, the center of the gyre moves closer to the northern wall, so that the northern boundary layer narrows. Meanwhile the circulation is enhanced, the maximum value of stream function $\psi_{\max }$ increases from 3.355 (7.235) to 4.552 (9.041) as $\lambda$ changes from 1 to 0.1 for $R_{a} \lambda^{2}=2 \times 10^{4}\left(2 \times 10^{5}\right)$ (table 3.2 and 3.3). All these suggest a swifter northern boundary layer current, which is mostly responsible for the northward shift of $b_{z}=0$ line.

It is interesting to note that the enhancement of the asymmetry of the circulation with decreasing $\lambda$ does not exceed a certain limit. Both asymmetry and intensity of the circulation approach this limit as $\lambda \leq 0.1$. This feature is robust regardless of the variation of $R_{a} \lambda^{2}$. In our numerical scheme, we solve $\nabla^{2} \psi=\psi_{z z}$ instead of $\nabla^{2} \psi=\lambda^{2} \psi_{y y}+\psi_{z z}$ when $\lambda=0.001$. The results are virtually unchanged between $\lambda=0.01$ and $\lambda=0.001$. This shows that our system behaves smoothly in the transition, and the circulation reaches a limit for an aspect ratio somewhere around 0.01. Below this value, the change in aspect ratio causes no change in the solution provided $R_{a} \lambda^{2}$ is fixed.

Special attention is paid to the temperature field and thermocline structure. It is seen from both figure (3.1) and (3.2) that as $\lambda$ decreases changes of the temperature field are very small; the interior is only slightly colder, and the region of unstable stratification narrows slightly, about one grid length to the north. There is no change in the thermocline, because a change of $\lambda$ with $R_{a} \lambda^{2}$ fixed does not change the balance between upwelling and downward diffusion in the interior region. Note that when $\lambda$ varies, it is $R_{a} \lambda^{2}$ not $R_{a}$ that is kept constant. As $\lambda$ changes from 1 to $0.001, R_{a}$ ranges from $2 \times 10^{4}$ to $2 \times 10^{10}$ in figure (3.1) and from $2 \times 10^{5}$ to $2 \times 10^{11}$ in figure (3.2). This means that in equation (3.1b) the coefficient in front of $b_{y}$ is constant in each set of experiments, and that allows us to compare the results at the same or different level of the forcing term. When we reduce $\lambda$ to the oceanic situation, we also increase the 
Rayleigh number to approach the true oceanic value. Now it is easy to understand why the thermocline does not change as $\lambda$ varies. As $\lambda$ is reduced, $R_{a}$ is increased; thus the strength of the surface buoyancy flux is increased by the same proportion. This stronger flux maintains the intensity of diffusion and upwelling over a wide region and thus the final balance between the two is unaltered.

It is interesting to see what happens as we keep $R_{a}$ fixed and vary only $\lambda$. As $\lambda$ decreases, $R_{a} \lambda^{2}$ also decreases, and we effectively reduce the importance of the horizontal buoyancy gradient in the vorticity equation. Since $R_{a} \lambda^{2} b_{y}$ is the only term that drives the vertical circulation, we would expect the circulation to decrease in intensity and the thermocline to deepen to balance a weakened upwelling. As the circulation becomes very weak, diffusion is predominant, and the temperature and the thermocline structures are hardly affected by the weak advection. The pattern approaches the purely diffusive limit (Nelken, 1987). These features are clearly seen in figure (3.3) for $R_{a}=2 \times 10^{4}$ and figure (3.4) for $R_{a}=2 \times 10^{5}$. Why does the circulation weaken and become symmetric when the Rayleigh number and thus the external thermal forcing is kept unchanged? When $R_{a}$ is fixed, the intensity of the surface buoyancy flux is the same, i.e., the peak heat flux entering in the south and leaving in the north are fixed, but it acts on a wider basin area as $\lambda$ becomes smaller. It is the horizontal buoyancy gradient, which is reduced as the basin is stretched horizontally, that contributes to the momentum field. As $\lambda$ decreases, $R_{a} \lambda^{2} b_{y}$ decreases quickly and the resulting circulation decreases and becomes more symmetric. Some typical values of the results are documented in table (3.4) for $R_{a}=2 \times 10^{4}$ and table (3.5) for $R_{a}=2 \times 10^{5}$. Even though the Rayleigh numbers are different in these two cases, the temperature field approaches the same limit value as $\lambda$ becomes small. This is not unexpected, because the Rayleigh number does not enter the buoyancy equation directly, and at the small $\lambda$ (weak advection) limit it reduces to the diffusion equation. 
We will study here in detail the cases of fixed values of $R_{a} \lambda^{2}$ as $\lambda$ varies. Later in this study, when we say that the aspect ratio is varying, we mean that $\lambda$ varies with $R_{a} \lambda^{2}$ fixed, except when otherwise indicated.

\subsection{On the Rayleigh Number}

It is undisputable that the Rayleigh number plays a crucial role in thermal convection problems. Consider a horizontal layer of fluid with an adverse temperature gradient imposed by cooling (heating) the top side (bottom side). Because of thermal contraction, the fluid at the cooled top will be heavier than the fluid at the bottom. This heavy surface stratification is statically unstable and there is a tendency for the fluid to redistribute itself. This tendency will be inhibited by viscosity. Rayleigh showed that the stability of such a layer of fluid is determined by the numerical value of the nondimensional parameter $R_{a}=g \alpha|d T / d z| D^{4} / \kappa \nu$, now known as the Rayleigh number. We have written it in conventional form using the vertical temperature gradient. Rayleigh further showed that instability must set in when $R_{a}$ exceeds a certain critical value $R_{a c}$; then a stationary pattern of motion will be set up. In essence, the Rayleigh number is a ratio of the destabilizing buoyancy force to the stabilizing viscous force. The Rayleigh number in oceanic problems is often of the order $10^{17}$, which is far larger than the critical Rayleigh number $\left(R_{a c}=10^{3}\right.$ to $10^{5}$ for a fluid sphere with a free surface. Chandrasekhar, 1961). Thus the ocean can not sustain even a slight vertical adverse temperature gradient (salinity effects are not included here). Convection occurs as soon as the adverse temperature gradient is imposed. Suppose we could impose a very small $|d T / d z|$, which reduces $R_{a}$ below the critical value. If we increase $|d T / d z|$ gradually, $R_{a}$ passes the critical value, and the fluid starts to move. This motion is usually laminar and steady for a certain range of $R_{a}$ above $R_{a c}$. If we keep increasing $R_{a}$, the intensity of the fluid motion will also increase, until $R_{a}$ is so large (e.g. $R_{a}>R_{a t}$ ) that the laminar and steady flow pattern of motion becomes turbulent. It is clear that 
the thermal convection process is closely related to the Rayleigh number. In this study the $R_{a}$ value is relatively small and falls safely into the regime of two-dimensional laminar convection (theoretically a two-dimensional convection may have higher $R_{a t}$ than a three-dimensional convection).

Previous studies of the thermally driven single cell circulation have focused heavily on the Rayleigh number dependence, so we will not go into a detailed analysis; instead we only summarize the important aspects of these results. In our numerical experiments, $R_{a}$ ranges from $2 \times 10^{4}$ to $2 \times 10^{12}$ for $\lambda=1$ to 0.001 . Some of our results have already been shown in figures (3.1) and (3.2) with $R_{a} \lambda^{2}=2 \times 10^{4}$ and $2 \times 10^{5}$ respctively. We can compare these results with ones having equal values of $\lambda$ but different $R_{a}$ 's, figure ( $3.1 \mathrm{~m}, \mathrm{n}$ and o) with $R_{a}=2 \times 10^{10}$ and figure $(3.2 \mathrm{~m}, \mathrm{n}$ and o) with $R_{a}=2 \times 10^{11}$, both at $\lambda=0.001$. The major influences of the Rayleigh number on the thermal and momentum fields are found to be:

(1) The intensity of the circulation increases as the Rayleigh number increases. $\psi_{\max }$ is about doubled when $R_{a}$ increases by a factor 10 . This tendency is the same for different values of $\lambda$.

(2) The vertical boundary layer narrows and the asymmetry increases as the Rayleigh number increases. Comparing figure $(3.1 \mathrm{n})$ with $(3.2 \mathrm{n})$, the former has a northern boundary layer width about $1 / 5$ th of the basin width, while the latter is only $1 / 10$ th of the basin width and the gyre center is more vertically asymmetric.

(3) The thermocline shallows and the area of the homogenized interior increases as the Rayleigh number increases.

(4) The variation of the buoyancy field decreases as the Rayleigh number increases, i.e., the buoyancy field tends to be more uniform at larger Rayleigh number. The values of $b_{\max }-b_{\min }$ are 0.374 for figure $(3.1 \mathrm{~m})$ and 0.265 for figure $(3.2 \mathrm{~m})$. 
(5) The area of the northern unstable region decreases as the Rayleigh number increases. The zero contour of $b_{z}$ is much closer to the northern wall in figure (3.2 o) than in figure (3.1 o).

(6) The system is diffusion dominated at small Rayleigh number. Advection becomes increasingly important as the Rayleigh number increases (figures (3.3) and (3.4)).

The Rayleigh number used in the present study is much larger than the values used by any previous investigators (table 3.1). Since we have also used small $\lambda$ at large $R_{a}$, we essentially have kept the value $R_{a} \lambda^{2}$ at about the same order of magnitude as that used by Beardsley and Festa (1972), and their cases are the same as ours with $\lambda=1$ (figures $3.1 \mathrm{a}, \mathrm{b}$ and $\mathrm{c}$, and $3.2 \mathrm{a}$. $\mathrm{b}$ and $\mathrm{c}$ ). The relationship between the Rayleigh number and the thermal and momentum fields will be further examined in section 3.7 where we will analyze the leading order balances in the system.

Numerical experiments are still limited to values of around $10^{6}$ for $R_{a} \lambda^{2}$, many orders of magnitude smaller than the $R_{a} \lambda^{2}$ for large scale geophysical problems. Laboratory experiments are severely limited by their aspect ratio; the highest $R_{a} \lambda^{2}$ achieved in Rossby's experiment is $2.56 \times 10^{9}$ with $\lambda=0.4$. However, in the laboratory, even at this Rayleigh number, instabilities may cause the onset of three-dimensional turbulence (Beardsley and Festa, 1972).

At the present stage, modelling at small $R_{a} \lambda^{2}$ values may provide valuable physical insight to understand the large scale thermally driven circulations. The ultimate goal of fully understanding this complicated process is beyond the scope of this study. 


\subsection{The Effects of Mixing}

In all the results presented in figures (3.1)-(3.4), the viscosity $\nu_{V}$ and the thermal diffusivity $\kappa_{V}$ in the vorticity and temperature equations are held constant. The resulting temperature field shows a typical unstable structure with heavy fluid overlying the light one at the northern part of the basin. In chapter two we introduced the idea of convective overturning mixing, which effectively reduces the gravitationally unstable density structure to a marginally stable or weakly unstable one; a result closer to reality. In this section we shall apply the same idea to thermally driven mixing and circulation. We will designate the case with constant values of $\kappa_{V}$ and $\nu_{V}$ the standard mixing case; it serves as a reference with which our results are compared. The other cases are the density overturning case and the density-momentum overturning case. The only distinction between the standard and the overturning cases is in the parameterization of $\kappa_{V}$ and $\nu_{V}$. The horizontal components of diffusivity and viscosity always have a constant value, $\bar{\kappa}_{H}=\bar{\nu}_{H}=1$.

\subsubsection{Standard Mixing Case}

By standard mixing we mean the conventional mixing due to the diffusive nature of the fluid. In section 3.4 we gave typical ranges of the dimensional values of $\kappa_{H}, \kappa_{V}$, $\nu_{H}$ and $\nu_{V}$ for oceanic problems. In the nondimensionalized equation (3.1) $\bar{\kappa}_{H}=\kappa_{V}=$ $\bar{\nu}_{H}=\nu_{V}=1$ for standard mixing case. The works cited in section 3.4 and 3.5 all used these values uniformly. Because the mixing rate is constant for both stable and unstable stratifications, the final results can have a statically unstable structure. 


\subsubsection{Density Overturning Mixing Case}

As in chapter two the vertical diffusivity is given by equation (2.4) with $\kappa^{+}=1$ and $\kappa^{-}=100$, but $\nu_{V}=1$ is kept constant, and $\gamma=-5 /\left|b_{z}\right|_{\max }$.

This is equivalent to assuming that the fluid is very diffusive in the unstable region and mixes quickly to reach a marginally stable state. The purpose of this parameterization is to simulate the effect of buoyancy forced turbulent convective mixing in the gravitationally unstable area, and therefore it does not mean physically the nature of the fluid in the unstable region differs from that in the stable region; thus we do not alter the overall Rayleigh number in the calculation and $R_{a}$ is still kept constant throughout the whole basin. We may define a so called local effective Rayleigh number based upon magnitude of $b_{z}, \kappa_{V}$ and $\nu_{V}$ in the overturned media; this effective Rayleigh number will certainly depend on the mixing parameterization. In this study we have used the previously defined constant Rayleigh number instead of the local effective Rayleigh number.

\subsubsection{Density-Momentum Overturning Mixing}

In the regions where the buoyancy structure is unstable, both density and momentum are mixed by convective overturning. Because the overturning mixing coefficients depend upon the vertical buoyancy gradient, momentum overturning can only occur in regions where the buoyancy structure is unstable. The value of $\nu_{V}$ is then exactly the same as $\kappa_{V}$. As the overturning mixing homogenizes the density of the fluid column, it also acts to redistribute the momentum field in the same manner. From the results of chapter two we saw that this method worked smoothly for the one-dimensional mixing problem. Its application to the two-dimensional mixing and convection problem follows the same form and procedure, except it is applied on many fluid columns at the same time. In the two dimensional case, the buoyancy and momentum fields are 
closely coupled through complicated interactions between the advection and diffusion processes. As in the density overturning case, the Rayleigh number is held constant.

\subsubsection{Results and Discussion}

Since we are primarily interested in thermal convection on a geophysical scale, we shall focus only on cases of small aspect ratio, typically $\lambda=0.001$. Two groups, $A$ and B, of cases are examined in great detail and are listed in table (3.6). Each group contains three different mixing cases as defined above at a fixed value of $R_{a} \lambda^{2}$. The results are characterized by $\psi_{\max }$, its location and the maximum and minimum values of buoyancy. The results corresponding to the groups A and B are plotted in figures (3.5) and (3.6), respectively. These figures present vertical sections of the results with the southern (heated) regions to the left and the northern (cooled) regions to the right. There are three columns in each figure, representing, from left to right, standard, density overturning and density-momentum overturning mixing cases. In each column, from top to bottom are contours of buoyancy, streamfunction and vertical buoyancy gradient. The curves extending from the top center to the lower left in the buoyancy and streamfunction fields are the contours of $\kappa_{V}$ and $\nu_{V}$ used in the overturning parameterization. The zero contour in the vertical buoyancy gradient represents the interface between the stable (south of the contour) and unstable (north of the contour) regions.

It is immediately obvious from the figures that the unstable buoyancy structure in the standard case has now vanished in both overturning mixing cases; instead, the isopycnals become almost vertical near the north wall, a result of strong vertical mixing there. The lack of the vertical mixing in the standard case causes cold water to be concentrated near the northern surface, forming a low temperature center there. The overturning mixing effectively brings this cold water down and results in an almost uniform fluid column with a higher near surface temperature. This is clearly seen by comparing $b_{\min }$ of two groups in table (3.6); here we must point out that $b_{\max }$ and $b_{\min }$ 
are located at the southern and northern top corners, respectively. Since the buoyancy field near the southern surface is nearly independent of the northern overturning, $b_{\max }$ is almost constant within each group. As a result the density overturning reduces the variation of the buoyancy field. This differs from the case examined by Nelken (1987). Nelken compared the standard case with density mixing case and found that the density overturning caused the isotherms to be more crowded near the surface. This is not present in our results. Because the domain in his study had an aspect ratio of one, the changes at the northern boundary affected the southern surface and significantly changed conditions there. In contrast, our basin is a thin flat one, with an aspect ratio 0.001 , and the southern surface is relatively insensitive to the changes occurring at the northern boundary.

The density overturning also affects the momentum field. Compared with the standard case, the northern boundary layer is broader so that the streamlines are less crowded next to the wall, and the circulation is slightly more intense, up by $3 \%$ for both groups A and B. The last feature is contradictory to Nelken's results which had a decreased circulation. Indeed, our results at a smaller $R_{a} \lambda^{2}$ value, $2 \times 10^{4}$, also shows a $4 \%$ decrease in $\psi_{\max }$ as we go from the standard to overturning mixing cases. We know that the circulation increases as $R_{a}$ increases as does the importance of the nonlinear advection terms in the vorticity equation. These nonlinear terms enter the dominant balance in the northern boundary layer (see section 3.7 for detail) and are crucial in transporting vorticity. At small $R_{a}$ and large $\lambda$ values, the boundary layer is much wider than that at large $R_{a}$ and small $\lambda$ values. The consequency is that nonlinear terms are more important for a narrow boundary layer (as in our case) than for a wide boundary layer (as in Nelken's case). In Nelken's study, the contribution to the vorticity field from advection terms is less than $5 \%$, and was thus neglected totally from vorticity equation. In his case $R_{a} \lambda^{2}=2 \times 10^{5}$ and $\lambda=1$, there is a very wide northern boundary layer, about $1 / 3$ of the basin width, and it might be a reasonable approximation for him to neglect the vorticity advection everywhere. Since 
the overturning mixing smooths out both horizontal and vertical buoyancy gradients in the northern mixing region, it effectively reduces $b_{y}$ and thus the circulation. In our case, $R_{a} \lambda^{2}=2 \times 10^{5}$ and $\lambda=0.001$, so that the boundary layer is only $1 / 10$ of the basin width; as we shall see in section (3.7), in the boundary layer the vorticity balance is between the vertical advection, horizontal diffusion and $b_{y}$. The decrease of $b_{y}$ due to overturning mixing interacts with the other two terms in such a manner that more potential energy is converted into kinetic energy, and the stronger advection is balanced by stronger diffusion. This results in an increase in circulation. The increase in $\psi_{\max }$ due to the density overturning is $3 \%$ for both groups $\mathrm{A}$ and $\mathrm{B}$, a value that is small enough not to distort the interior and the thermocline appreciably away from the standard cases; a very slight upward shift of the thermocline can barely be seen in the $b_{z}$ contours.

The effects of overturning of both density and momentum compared with overturning of density are seen mainly in the momentum field. The center of the vertical gyre is pushed downward one or two grid points by the convective momentum mixing. The streamlines are less crowded against the north wall and are almost evenly spaced across the boundary. The circulation is slightly increased, again a contribution due to the redistribution of the energy and the interaction between the advection and the enhanced diffusion processes in the overturning region. The influence of the overturning mixing does not extend to the interior and the thermocline; both the circulation pattern and the buoyancy structure are the same as before. A small upshift of the contour $b_{z}=0.1$ reflects the increase in circulation due to overcoming downward diffusion. As pointed out by Killworth (1976), there is little change of the density field as momentum is mixed in the same manner as density. From those results we see that this is indeed the case for the buoyancy field both in and out of the overturning region; but the effect on the momentum field is more pronounced. 
To further understand the overturning effect, we examine the energy exchange process during mixing. Since $b$ is a conserved quantity, the mixing simply redistributes it, or reduces its variation, $\Delta b$. In the present case, because the net heat flux into the system is zero, the total density of the system must be conserved. The release of potential energy due to mixing the upper heavy fluid with the lower light ones is balanced by an increase in kinetic energy. In the overturned region there are two distinct releases of potential energy occurring simultaneously: (1) a vertical redistribution of buoyancy until the stratification is marginally stable, and (2) horizontal stratification tends to adjust to a state of minimum available potential energy. Potential energy is rapidly redistributed through vertical overturning mixing. At steady state the energy level of a fluid column does not change; potential energy released by mixing unstable stratification is thus converted to kinetic energy. In the adjustment process the buoyancy field reaches a state of uniform or small horizontal stratification which corresponds also to the uniform vertical stratification, a state of minimum available potential energy. The potential energy released during this adjustment process also becomes kinetic energy. The combined contributions from both processes are not significant in maintaining the circulation; compared with the standard cases, $\psi_{\max }$ is up by $5 \%$ in group $\mathrm{A}$ and $12 \%$ in group B, when both density and momentum overturning mixing are involved.

As an aside, we remark on the formation of a weak counterclockwise circulation below the main gyre in figure (3.6). It is located in the lower southern part of the basin. The new circulation is totally in the interior region of almost homogeneous density, and its existence is independent of the mixing parameterization. It is obvious that the circulation is shaded by the main gyre from direct influence of the surface buoyancy flux and yet also seems to be a direct result of the increase of the Rayleigh number, i.e., the intensity of the surface flux in our definition. The only difference between the corresponding cases in groups A and B is the Rayleigh number, $2 \times 10^{11}$ in group A and $2 \times 10^{12}$ in group B. The group B has the highest Rayleigh number used in this study, also the highest ever used in any of the previous studies, numerical or laboratory. 
The higher Rayleigh number means a more nonlinear momentum field, especially in the northern boundary region. The extremely narrow northern boundary layer corresponds to a very strong downward jet. It flows fast along the northern boundary and suddenly turns into the interior. The inertial effect of this flow is probably responsible for its sharp upward-turning in flow direction and leaves the rest of the interior dominated by $R_{a} \lambda^{2} b_{y}$ and vertical diffusion. Examination of the term $b_{y}$ shows that the horizontal buoyancy gradient reverses its sign from the surface to the deep water for case B. The magnitude of $b_{y}$ is very small in the lower interior of the basin, about $2 \%$ of the magnitude of $b_{y}$ at surface, but it may be responsible for driving the weak clockwise deep circulation in that region. We are not sure how this deep circulation depends upon the frictional boundary condition. Future work with the stress boundary condition on this problem may be helpful in understanding the complication of the circulation pattern. More work with higher Rayleigh number is also needed to confirm and further understand this problem.

\subsection{Analysis on the Dominant Balances of the System}

The most striking feature in the results presented above (figure 3.1, 3.2, 3.5 and 3.6) is the existence of boundary layers in both the thermal and the momentum fields. The thermal boundary layer next to the non-insulated boundary has isotherms densely packed near the surface, while the dynamic boundary layer is a downward jet next to the northern insulated wall. These boundary layers are clearly associated with advective processes, almost disappearing as the solution approaches the diffusive limit at small $R_{a} \lambda^{2}$ (figure $3.3,3.4$ ).

As we emphasized in previous sections, the asymmetry of the buoyancy and the momentum fields is a key feature to this problem. Imagine that we start from zero fields for both buoyancy and stream function and impose a weak heat flux at 
surface. The heat flux is so weak that the redistribution of buoyancy is mainly a diffusive process. The circulation due to this horizontal nonhomogeneity is too weak to balance the diffusion, and the system is virtually governed by the right hand side of equation $(3.1 \mathrm{~b}, \mathrm{c})$. Thus both the buoyancy and the momentum fields are symmetric, as shown in figure $(3.3 \mathrm{~g}, \mathrm{~h}, \mathrm{i})$ and $(3.4 \mathrm{~g}, \mathrm{~h}, \mathrm{i})$. The existence of the weak circulation field is simply the result of the presence of the horizontal buoyancy gradient. To the lowest order there is no interaction between these two fields. As the strength of the forcing, or equivalently, $R_{a} \lambda^{2}$ increases, the circulation increases and advection becomes important in the buoyancy equation everywhere. Since advection is far more effective than diffusion in transporting heat, the isotherms must be closely packed to allow diffusion to balance the upward advection over a large part of the interior. It is apparent that the stronger the advection, the closer the isotherms are pushed toward the surface, and thus the shallower the thermocline. This forms the asymmetric thermal field.

Since advection is much more efficient than diffusion in heat transport, the balance between these two processes must take place in a large area. If $R_{a} \lambda^{2}$ is reasonably large, both advection and diffusion are important, and as we already have seen from previous results, the interior motion is dominantly upward. This means the momentum field is asymmetric relative to the basin center line. From continuity, if fluid is brought up everywhere in the interior, it must go down along one of the vertical boundaries. This boundary layer must be next to the north wall for a simple reason. It has to obey the second law of thermodynamics; if it were next to the south wall, it must flow against the density gradient along that boundary, i.e., advecting light fluid under heavy ones. That violates the second law and is impossible for this problem. From a mathematical point of view, we can find solutions to the linearized boundary layer equation at either south or north wall (the vorticity equation can be approximately written as a fourth order linear ODE about stream function), and both solutions vary exponentially across the boundary layer but only the one at the northern boundary 
remains finite as it extends to the interior. This excludes the possibility of existence of a southern boundary layer.

The surface and northern boundary layers are fundamentally different. The horizontal one is a thermal boundary layer, characterized by strong vertical temperature gradient above a homogeneous interior. Inside this layer, the flow almost follows the isolines before they become vertical. This is not altered by the implementation of the convective overturning. The vertical boundary layer is a dynamic boundary layer, with high velocity and large shear. It is very sensitive to the mixing parameterization, which may widen the boundary layer and convert more available potential energy to kinetic energy, thus increasing the total circulation. The two boundary layers are closely related to each other through interaction of diffusive and advective processes. It is the fluid in the thermocline that turns into the northern jet. It is an obvious fact that one boundary layer can not exist without the existence of the other. Actually it is easy to prove that, from the existence of any one of the two boundary layers, there must be another boundary layer in the system.

Analyses for the existence and the location of the boundary layers have been made. The order of magnitude have also been estimated, such as the width of the boundary layer, the magnitude of the stream function and the temperature difference across the boundary layer. The analyses take advantage of the large scale difference in the directions along and across the boundary layer, and thus reduce the full equations to simple balances of the leading order terms. The balances relate the major unknowns to the known quantities, usually the Rayleigh number and the surface heat flux or temperature, and give us a direct and simple picture about the dominant processes and the fundamental mechanisms in the system.

The estimates of the relevant quantities in the thermocline and the northern jet have been given previously by Rossby (1965) and Nelken (1987). Their analyses give an order of magnitude for the stream function and the boundary layer thickness 
in terms of Rayleigh number and the surface forcing. They dropped nonlinear terms in the vorticity equation and did their analysis without consideration of convective overturning. The aspect ratio, an important element as we already have seen in section (3.4), is relatively large in these studies (see table 3.1). Here we shall examine this problem considering convective overturning, vorticity advection and a wide range of aspect ratios. We shall compare our analysis with the results of the numerical solution of equation (3.1).

In the thermocline, we replace the vertical coordinate $z$ by a stretched boundary layer coordinate $\eta=z / d_{T}$, where the scale thickness of the thermocline $d_{T} \ll 1$. It is important to remember that all variables in equation (3.1) are already dimensionless, e.g., $z$ is the dimensionless depth scaled by the basin depth $D$ and therefore varies between $(0,1)$. So here $d_{T}$ is only a number, characterizing the dimensionless thickness of the thermocline; the dimensional thickness is $d_{T} D$. This is a thermal boundary layer, so that $\psi$ still varies over the whole depth, and the scaling balance from equation (3.1 $\mathrm{b}$ and $\mathrm{c})$ is

$$
\begin{array}{r}
R_{a} \lambda^{2} \Delta B_{T}=\left(\lambda^{2} \Psi_{T}+\frac{\Psi_{T}}{d_{T}^{2}}\right)+\nu_{V}\left(\lambda^{2} \frac{\Psi_{T}}{d_{T}^{2}}+\frac{\Psi_{T}}{d_{T}^{4}}\right) \\
\Psi_{T} \Delta B_{T}+\frac{\Psi_{T} \Delta B_{T}}{d_{T}}=\Delta B_{T}+\kappa_{V} \frac{\Delta B_{T}}{d_{T}^{2}}
\end{array}
$$

where we temporarily drop the vorticity advection in the first equation and shall return to it later. $\psi$ and $b$ are rescaled by $\Psi_{T}$ and $\Delta B_{T}$, their magnitudes in the thermocline. $\nu_{V}$ and $\kappa_{V}$ are of order one away from the overturning region. The leading order balance in the thermocline is

$$
\begin{aligned}
R_{a} \lambda^{2} \Delta B_{T} & =\frac{\Psi_{T}}{d_{T}^{4}} \\
\frac{\Psi_{T} \Delta B_{T}}{d_{T}} & =\frac{\Delta B_{T}}{d_{T}^{2}}
\end{aligned}
$$

which corresponds to the dimensional balance from (1.2)

$$
b_{y}+\nu_{V} \frac{\partial^{4} \psi}{\partial z^{4}}=0
$$




$$
\psi_{y} b_{z}-\kappa_{V} \frac{\partial^{2} b}{\partial z^{2}}=0
$$

With our specification of order one dimensionless surface buoyancy flux, $\Delta B_{T} / d_{T}=1$, equation (3.3) leads to the power law

$$
\begin{aligned}
\Psi_{T} & =\left(R_{a} \lambda^{2}\right)^{\frac{1}{6}} \\
d_{T} & =\left(R_{a} \lambda^{2}\right)^{-\frac{1}{6}} \\
\Delta B_{T} & =d_{T}
\end{aligned}
$$

It is interesting to note that the stream function (or the strength of the circulation) is inversely proportional to the buoyancy difference in the thermocline from this simple analysis. This agrees with the decreased variation of the buoyancy field as $R_{a} \lambda^{2}$ (and thus the circulation) increases. The decrease of $\Delta B_{T}$ requires the thermocline to shallow so that the surface flux is kept of order one.

Now we return to the vorticity advection in the thermocline. If we suppose that the power law of (3.4) is still correct in the presence of $J\left(\psi, \nabla^{2} \psi\right)$, the magnitude of vorticity advection (substituting (3.4) into the Jacobian), gives

$$
J\left(\psi, \nabla^{2} \psi\right) \sim\left(R_{a} \lambda^{2}\right)^{\frac{5}{6}}
$$

which is exactly the same order as the right hand side of (3.1b). This is not to say that $J\left(\psi, \nabla^{2} \psi\right)$ is always as important as the terms $R_{a} \lambda^{2} b_{y}$ and $\nu_{V} d^{4} \psi / d z^{4}$ in the thermocline (at least for small $R_{a} \lambda^{2}, J\left(\psi, \nabla^{2} \psi\right)$ is unimportant). This analysis tells us one cannot simply neglect vorticity advection without further justification, especially when $R_{a} \lambda^{2}$ is large. For $R_{a} \lambda^{2}=2 \times 10^{5},(3.4)$ gives $\Psi_{T}=7.65, d_{T}=0.13$. The first value agrees well with the numerical solution. If we define thermocline depth in the numerical simulation to be the $e^{-1}$ decay depth of $b_{z}, d_{T}$ also agrees well with it.

In the northern jet we rescale $\psi$ and $b$ by $\Psi_{N}$ and $\Delta B_{N}$, here $\Delta B_{N}$ is the buoyancy difference across the jet. As in the thermocline, we introduce stretched 
boundary layer coordinate $\xi=y / l_{N}$. The first order buoyancy and vorticity equations (in magnitude) in the northern boundary layer are

$$
\begin{aligned}
R_{a} \lambda^{2} \frac{\Delta B_{N}}{l_{N}} & =\frac{\Psi_{N}}{l_{N}^{2}}\left(\frac{\lambda^{2}}{l_{N}^{2}}+1\right)+\nu_{V} \Psi_{N}\left(\frac{\lambda^{2}}{l_{N}^{2}}+1\right) \\
\frac{\Psi_{N} \Delta B_{N}}{l_{N}} & =\frac{\Delta B_{N}}{l_{N}^{2}}+\kappa_{V} \Delta B_{N}
\end{aligned}
$$

where we included $\nu_{V}$ and $\kappa_{V}$ because they can be large in the overturning region. Since the overturning mixing smooths out the vertical gradient, the vertical diffusion terms in the vorticity and buoyancy equations do not increase proportionally as $\nu_{V}$ and $\kappa_{V}$ do; instead the vertical uniformity makes vertical diffusion less important than horizontal diffusion. Once the vertical homogeneity is achieved, the overturning is confined only to a small portion near to the coldest corner. This is clearly seen from previous numerical results. Therefore the vertical diffusion may enter the balance but it certainly does not change the overall dominant balance in the boundary layer. To the leading order

$$
\begin{aligned}
R_{a} \lambda^{2} \frac{\Delta B_{N}}{l_{N}} & =\frac{\Psi_{N}}{l_{N}^{2}}\left(\frac{\lambda^{2}}{l_{N}^{2}}+1\right) \\
\frac{\Psi_{N} \Delta B_{N}}{l_{N}} & =\frac{\Delta B_{N}}{l_{N}^{2}}
\end{aligned}
$$

or in dimensional form

$$
\begin{aligned}
b_{y}+\nu_{H}\left(\nabla^{2} \psi\right)_{y y} & =0 \\
J(\psi, b)-\kappa_{H} b_{y y} & =0
\end{aligned}
$$

where we again dropped the nonlinear terms in the vorticity equation for the simplicity of analysis. (3.5b) relates the stream function to the width of the jet,

$$
\Psi_{N}=\frac{1}{l_{N}}
$$

Now there are three possible balances for the vorticity equation (3.5a), depending on the value of $\left(\lambda / l_{N}\right)^{2}$.

$$
\text { i) }\left(\lambda / l_{N}\right)^{2} \gg 1
$$


An example is the case $\lambda=1$. The dominant balance gives

$$
\Delta B_{N}=R_{a}^{-1} l_{N}^{-4}
$$

since the fluid in the jet is from the thermocline, we shall use $\Psi_{N}=\Gamma \Psi_{T}$. If $\Gamma=1$, the transport in the thermocline equals that of the jet; if $\Gamma>1$, only part of the flow in the jet is from the thermocline. The balance yields

$$
\begin{aligned}
\Psi_{N} & =\Gamma\left(R_{a} \lambda^{2}\right)^{\frac{1}{6}} \\
\Delta B_{N} & =\Gamma^{4} \frac{\left(R_{a} \lambda^{2}\right)^{\frac{2}{3}}}{R_{a}}
\end{aligned}
$$

If $\Gamma=1, R_{a} \lambda^{2}=2 \times 10^{5}$ and $\lambda=1$, this gives $\Psi_{N}=7.65, \Delta B_{N}=0.017$ and $l_{N}=0.13$. The temperature gradient across the jet $\Delta B_{N} / l_{N}=0.13$, is about one third of the basinwide horizontal temperature gradient.

ii) $\left(\lambda / l_{N}\right)^{2} \ll 1$

The typical example is the case $\lambda=0.001$. The cross boundary layer temperature difference is

$$
\Delta B_{N}=\frac{1}{R_{a} \lambda^{2} l_{N}^{2}}
$$

We still assume $\Psi_{N}=\Gamma \Psi_{T}$, and $\Gamma=1$, i.e., all the fluid entering the jet is from the thermocline. For $R_{a} \lambda^{2}=2 \times 10^{5}$ and $\lambda=0.001$, this yields $\Psi_{N}=7.65, l_{N}=0.13$ and $\Delta B_{N}=2.96 \times 10^{-4}$. Both $\Psi_{N}$ and $l_{N}$ are quite close to the value given by the numerical results. Notice that $\Psi_{N}$ and $l_{N}$ are relatively insensitive to $\lambda$ as long as $R_{a} \lambda^{2}$ is kept constant, but $\Delta B_{N}$ decreases greatly. Now $\Delta B_{N} / l_{N}=2.28 \times 10^{-3}$, which is only about one hundredth of the horizontal temperature gradient over the whole basin.

iii) $\left(\lambda / l_{N}\right)^{2} \sim 1$

The example for this is case $\lambda=0.1$. The dominant balance in this case is the same as that in (ii). By examining figure (3.1) and (3.2) carefully, we can see the cases with $\lambda=0.1$ are very similar to the cases with $\lambda=0.01$ and 0.001 . 
In all three possible balances, the dimensional expressions have the same form. In the buoyancy equation the lateral diffusion is balanced by downward advection of $b$; the balance is not affected by the varying aspect ratio. The northern boundary layer always has a balance between the horizontal buoyancy variation and the lateral vorticity diffusion,

$$
\begin{aligned}
b_{y}+\bar{\nu}_{H}\left(\nabla^{2} \psi\right)_{y y} & =0 \\
\psi_{y} b_{z}-\bar{\kappa}_{H} b_{y y} & =0
\end{aligned}
$$

where $\nabla^{2} \psi=\lambda \psi_{y y}+\psi_{z z}$ is reduced to $\nabla^{2} \psi=\psi_{z z}$ for small value of $\lambda$.

In above analysis, we have deliberately dropped $J\left(\psi, \nabla^{2} \psi\right)$ from the vorticity equation. We already indicated in the previous sections that the nonlinear advection of vorticity is important in the thermal jet, so it must be included in the balance analysis. If we use the same scalings from above analysis, the Jacobian in the northern boundary layer is

$$
J\left(\psi, \nabla^{2} \psi\right) \sim \frac{\Psi_{N}^{2}}{l_{N}}\left(\frac{\lambda^{2}}{l_{N}^{2}}+1\right)
$$

from (3.5b), $\Psi_{N}=1 / l_{N}$; thus this has exactly the same order of magnitude as the terms in (3.5a), regardless of the value $\left(\lambda / l_{N}\right)^{2}$. Again we have to emphasis that this analysis only indicates the possible importance of $J\left(\psi, \nabla^{2} \psi\right)$; further justification is needed when one tries to linearize the vorticity equation.

The values of the individual terms in the buoyancy and vorticity equations are presented in figure (3.7) to (3.9). In (3.7.1), (3.8.1) and (3.9.1) the terms $v b_{y}, w b_{z}, \bar{\kappa}_{H} b_{y y}$ and $\left(\kappa_{V} b_{z}\right)_{z}$ are plotted as a function of depth at different latitudes. The curves are marked by the characters $\mathrm{a}, \mathrm{b}, \mathrm{c}$ and $\mathrm{d}$ corresponding to above four terms, respectively. The profiles in the four panels (i), (ii), (iii), (iv) are taken at latitudes $6 / 64,30 / 64,50 / 64$ and 62/64, respectively; where latitude 0 corresponds to the south wall and 1 to the northern extent of the domain. The vertical axis of each plot is depth, and varies from 0 at bottom to 32 at surface. Similarly in (3.7.2) to (3.9.2), $v\left(\nabla^{2} \psi\right)_{y}, w\left(\nabla^{2} \psi\right)_{z}, R_{a} \lambda^{2} b_{y}$, 
$\bar{\nu}_{H}\left(\nabla^{2} \psi\right)_{y y}$ and $\left[\nabla \cdot\left(\nu_{V} \nabla \psi_{z}\right)\right]_{z}$ are plotted at the same four latitudes against depth, and are marked by a-e, respectively. For the particular cases shown here, $R_{a} \lambda^{2}=2 \times 10^{6}$, $\lambda=0.001$, with different mixing parameterizations: figure (3.7) is standard, (3.8) has density overturning only and (3.9) has both density and momentum overturning. The smaller inserted picture in each figure shows the same plot but only for the portion from bottom to the base of the thermocline.

We first examine figure (3.7.1)-(3.9.1). All the terms are small in the lower half of the domain throughout the basin. The balance in the thermocline $(z>25)$ is very distinct, with large variations of both advection and diffusion, reflecting the predominant influence of the direct surface flux. In the region below the thermocline, the dominant balance is clearly between the vertical advection $w b_{z}$ and the vertical diffusion $\left(\kappa_{V} b_{z}\right)_{z}$, with small horizontal advection and diffusion except at latitude 62/64, which is located in the northern downward jet. Inside the jet, the balance is between the vertical advection and horizontal diffusion $\bar{\kappa}_{H} b_{y y}$. This is true for all standard and overturning cases, but the balance is more obvious with the presence of the convective mixing. It is clearly observed in the overturning cases, figure (3.8.1)iv and (3.9.1)iv, that the terms (b) and (c) dominate the balance below the depth where the curves (b) and (c) intersect the curves (a) and (d). The depth of this intersection is the depth that convective mixing can reach at that latitude. Above this depth, vertical convective mixing is strong and must be balanced by horizontal advection. The effect of convective overturning on the balance of the buoyancy field is confined mainly to the region near to the northern boundary; its influence on the balance outside this region is very small.

The balance in the vorticity equation is quite complicated. Like the buoyancy equation, individual terms are small in the lower half of basin and become drastically larger in the thermocline, figure (3.7.2) to (3.9.2). But below the thermocline the variation of individual terms is gentle and slow. Unlike the buoyancy equation there is no obvious two term dominant balance in the interior for the vorticity equation. Careful 
examination of figure (3.7.2) to (3.9.2) reveals that below the thermocline the balance is actually among three terms, $w\left(\nabla^{2} \psi\right)_{z}, R_{a} \lambda^{2} b_{y}$ and $\left[\nabla \cdot\left(\nu_{V} \nabla \psi_{z}\right)\right]_{z}$, with small horizontal advection and diffusion of vorticity. This generally agrees with our previous analysis about the vorticity equation, and especially the argument about the vorticity advection, which clearly enters the leading order balance here. In the northern jet, we observe a balance between vertical advection and horizontal diffusion. This is not totally surprising even though it somewhat differs from our previous scale analysis. In the jet, the horizontal temperature gradient is so small that the jet is no longer driven directly by this term; meanwhile the vertical advection of vorticity becomes increasingly important as the Rayleigh number becomes large. Now in the northern boundary layer both the buoyancy equation and vorticity equation are dominated by the advection-diffusion processes, i.e., the balance is only between the horizontal diffusion and the vertical advection. With this balance we can go through the same scale analysis as before and get only one relation $\Psi_{N}=1 / l_{N}$ for both buoyancy and vorticity balance equations. This is valid only for cases that have large enough $R_{a} \lambda^{2}$ value. Now $\Delta B_{N}$ does not enter the dominant balance and can not be estimated by this simple scale analysis. However our previous analysis about the northern boundary layer holds approximately for $\Psi_{N}$ and $l_{N}$ (since $J\left(\psi, \nabla^{2} \psi\right)$ is still of the same order of magnitude as $\kappa_{H} \nabla^{2} \psi_{y y}$ there), but the estimate for $\Delta B_{N}$ can only be regarded as an upper limit for the buoyancy difference across the northern jet. If $R_{a} \lambda^{2}$ is small and vorticity advection is less important compared with diffusion in the northern boundary layer, our previous scale analysis will give good estimates for the quantities in the boundary layer.

The effect of momentum overturning is mainly confined to the depth the overturning can reach. Below this depth, the situation is the same as the density overturning case. Inside the overturning region, the individual terms vary quite erratically with enormously large changes, figure (3.9.2)iii, iv. These noisy terms, though large, managed to cancel each other and converge to a steady solution. This behavior is most probably caused by the vertical diffusion term, which with the overturning parameter- 
ization can change drastically with depth for a relatively rough $\nabla^{2} \psi$ field. Fortunately this enormously noisy behavior appears only locally in the overturning region and does not contaminate the neighboring area.

\subsection{Summary}

Due to the fact that in oceanic problems the values of $\kappa_{H}$ and $\nu_{H}$ change for different time and space scales of motion, it is not justified that one can totally neglect horizontal diffusion in problems of large horizontal scale. Instead the horizontal diffusion may be important under certain circumtances. Here we have shown that horizonal diffusion can be locally dominant in the northern boundary layer; but in the interior of the basin it is negligible compared with vertical diffusion.

With $R_{a} \lambda^{2}$ value fixed, the change of $\lambda$ has a pronounced effect on the system. It causes an increase in asymmetry and circulation, resulting in a narrower and swifter cold downward jet next to the northern boundary compared to $\lambda=1$. The system is independent of $\lambda$ for $\lambda \leq 0.01$.

The controlling parameter for the system is the Rayleigh number. At small $R_{a}$, the system is diffusion dominated; there is no asymmetry in either buoyancy and circulation field. As $R_{a}$ increases, the circulation and asymmetry increase correspondingly, and the northern jet becomes increasingly narrow. The interior is almost uniform in density. Both diffusion and advection are involved in the dominant balance of the system. Since the northern boundary layer is extremely narrow and nonlinear at high Rayleigh number, it poses difficulties for numerical simulations. It is known that instability in circulation will eventually occur if $R_{a}$ is large enough. It was observed in laboratory experiments (Beardsley and Festa, 1972) that three dimensional turbulence occurred at $R_{a} \geq 10^{9}$ for $\lambda=0.4$. This critical Rayleigh number is still several order of magnitude smaller than the common oceanic values. Our present simulations have 
not seen such instabilities. This is perhaps due to fact the instability is often related to three-dimensional evolution of field structure, which is lacking in our two-dimensional model.

The parameterization of convective mixing eliminates the obvious unstable density structure. It acts to convert available potential energy to the kinetic energy and therefore increases the circulation intensity. The turbulent momentum mixing also contributes to the circulation field and tends to move the center of the circulation downward. In general, the interior structure of buoyancy and circulation is not significantly affected by the turbulent mixing parameterization.

The scale analysis of the system shows qualitative agreement with the numerical simulation. The distinct balances in different boundary layers stand out as the characteristic structure of the system. The depth of the thermocline from the scale analysis is quite shallow compared with oceanic thermocline. This indicates that the interior upwelling is too strong in the model, which may not be the case in a rotational framework. With rotation, the major part of the energy goes to maintain the thermal wind circulation in the horizontal plane, and the resulting vertical circulation will probably be much weaker. 

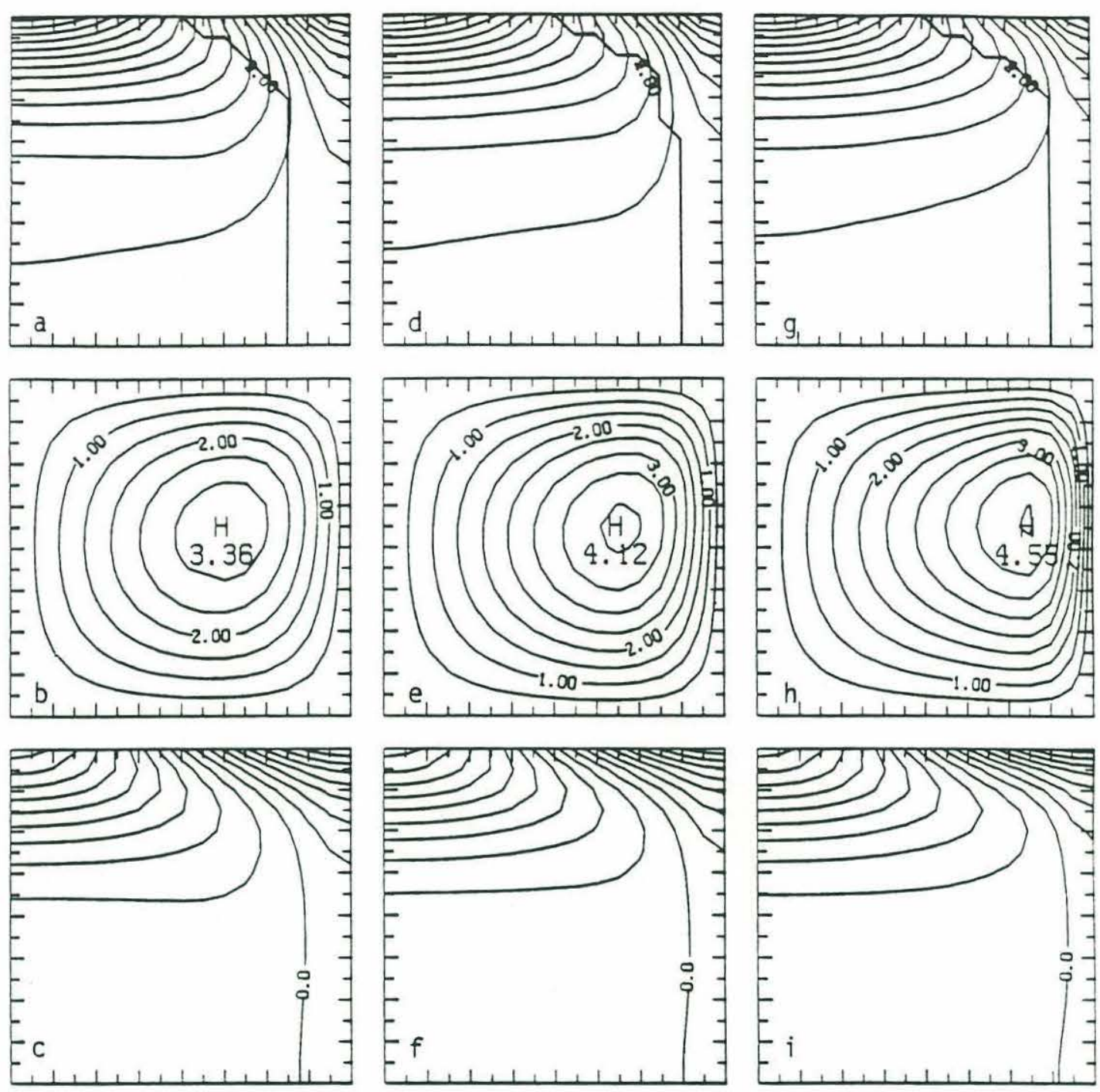

Figure 3.1: The steady state contours of $b(\mathrm{a}, \mathrm{d}, \mathrm{g}, \mathrm{j}$ and $\mathrm{m}), \psi(\mathrm{b}, \mathrm{e}, \mathrm{h}, \mathrm{k}$ and $\mathrm{n}$ ) and $b_{z}$ (c, f, i, 1 and o). $R_{a} \lambda^{2}=2 \times 10^{4}$ is fixed. $\lambda=1,0.5,0.1,0.01$ and 0.001 for the columns from left to right. 

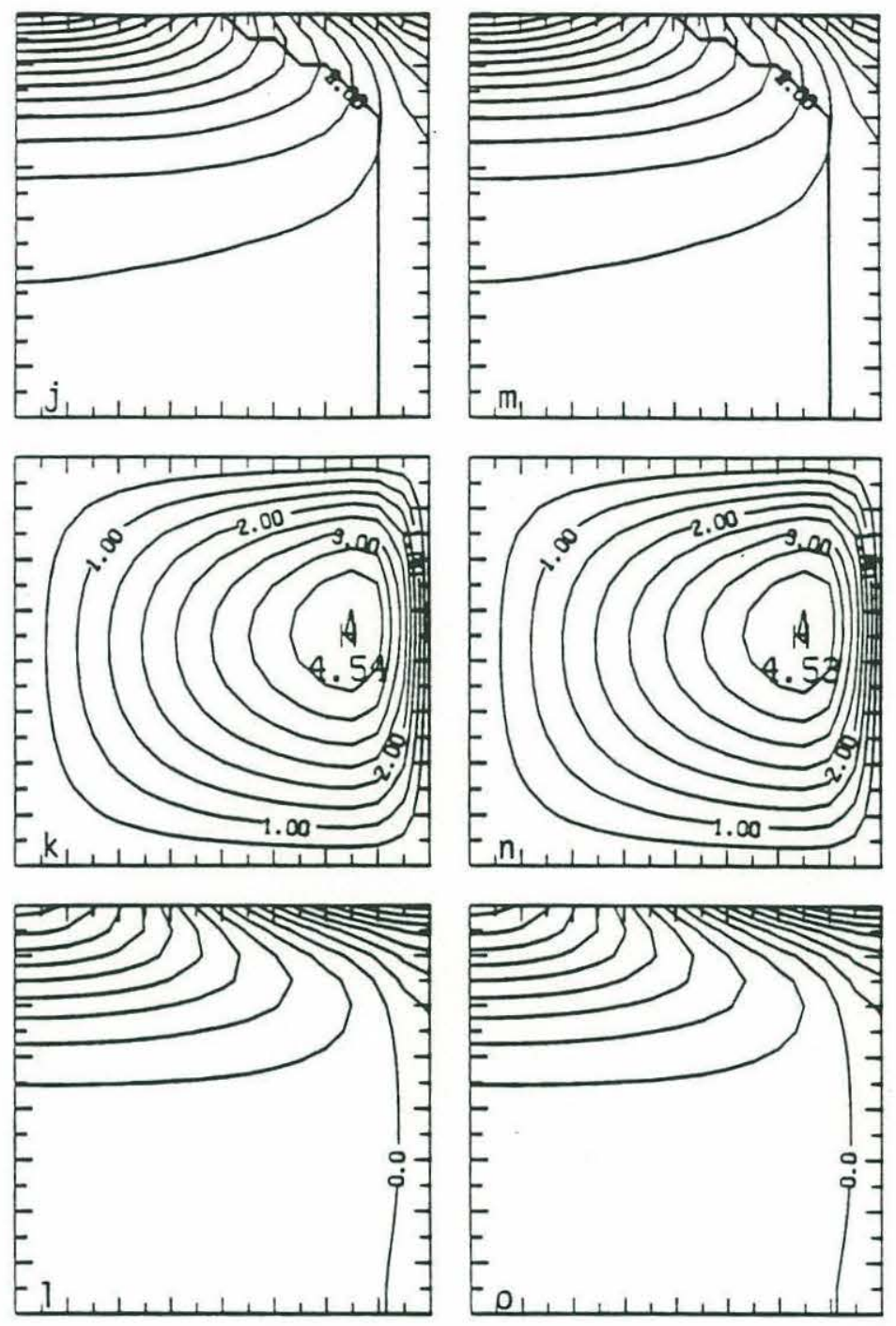

Figure 3.1: Continued. 

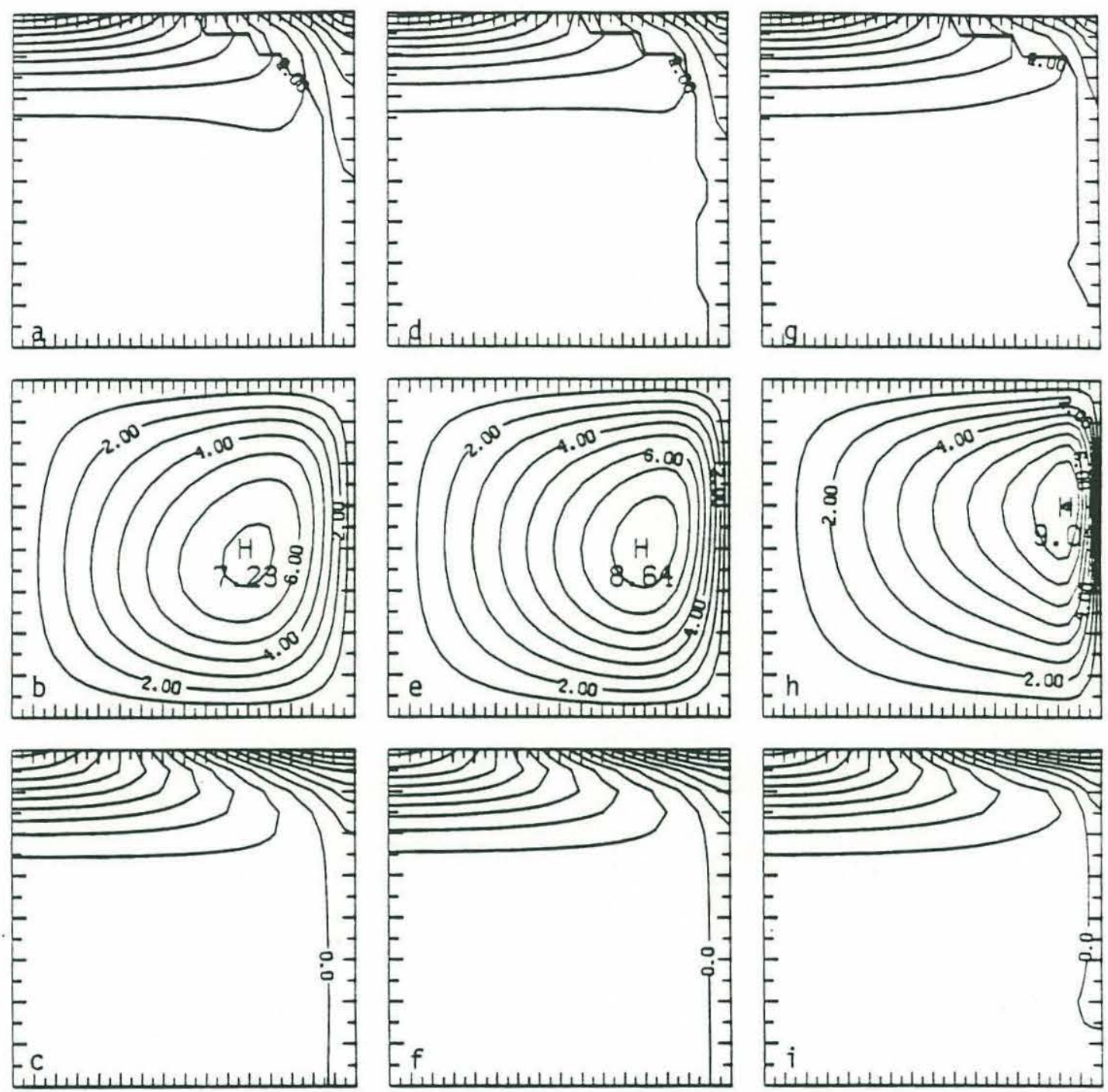

Figure 3.2: The same as figure 3.1, but for $R_{a} \lambda^{2}=2 \times 10^{5}$. 

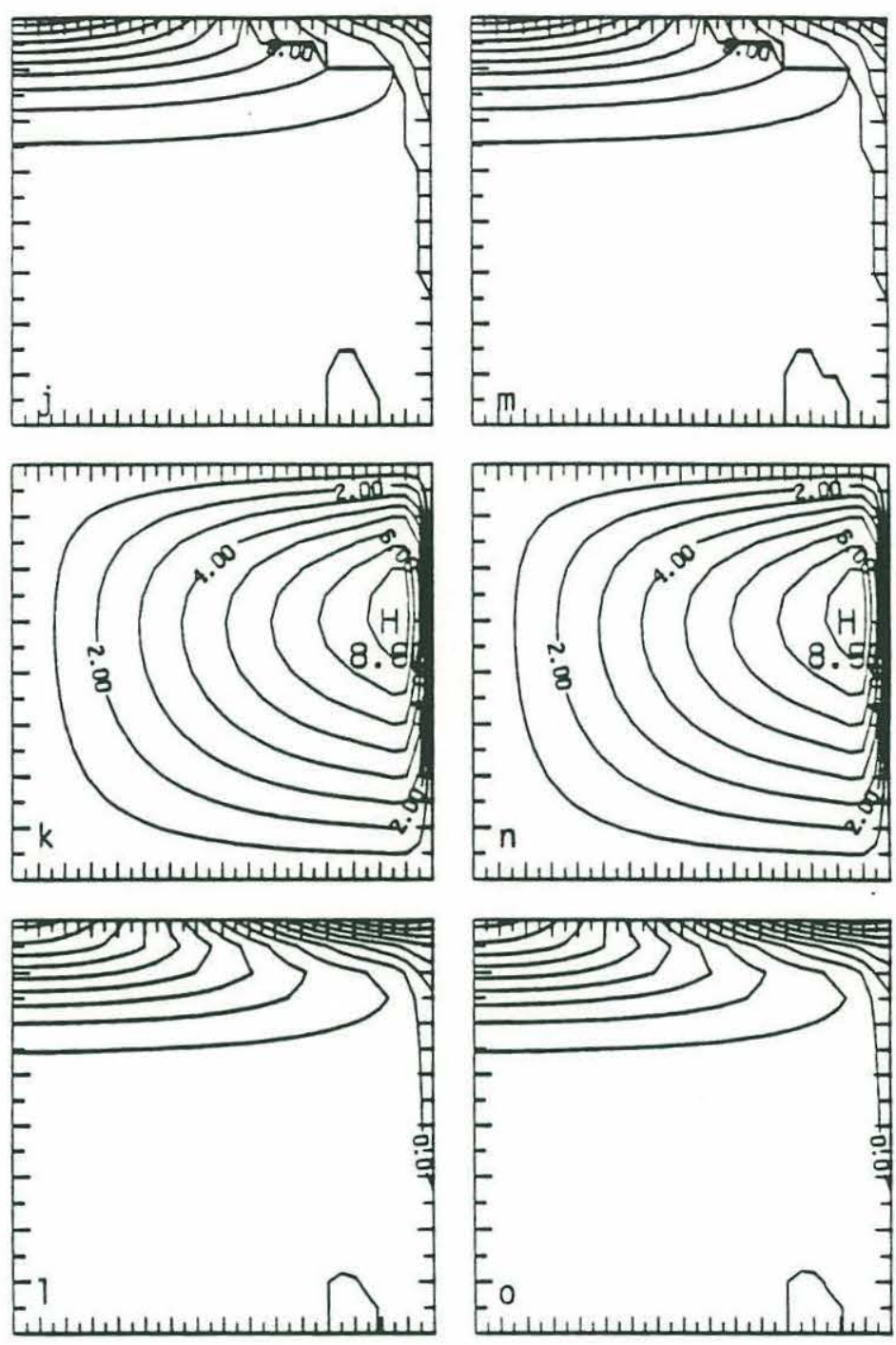

Figure 3.2: Continued. 

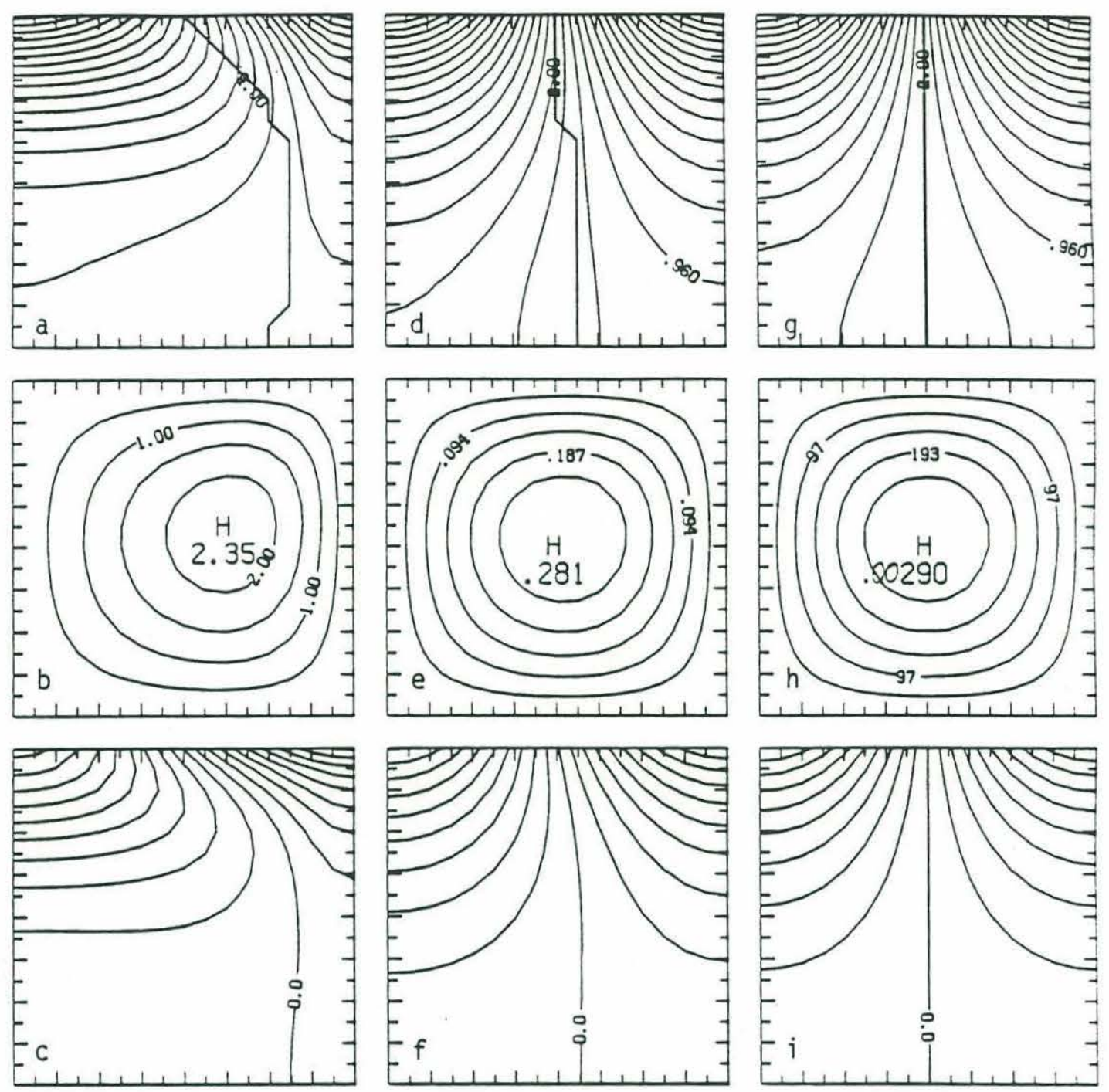

Figure 3.3: The steady state contours of $b$ (a, d and $\mathrm{g}$ ), $\psi\left(\mathrm{b}, \mathrm{e}\right.$ and $\mathrm{h}$ ) and $b_{\boldsymbol{z}}$ (c, f and i). $R_{a}=2 \times 10^{4}$ is fixed. $\lambda=0.5,0.1$ and 0.01 for the left, middle and right columns, respectively. 

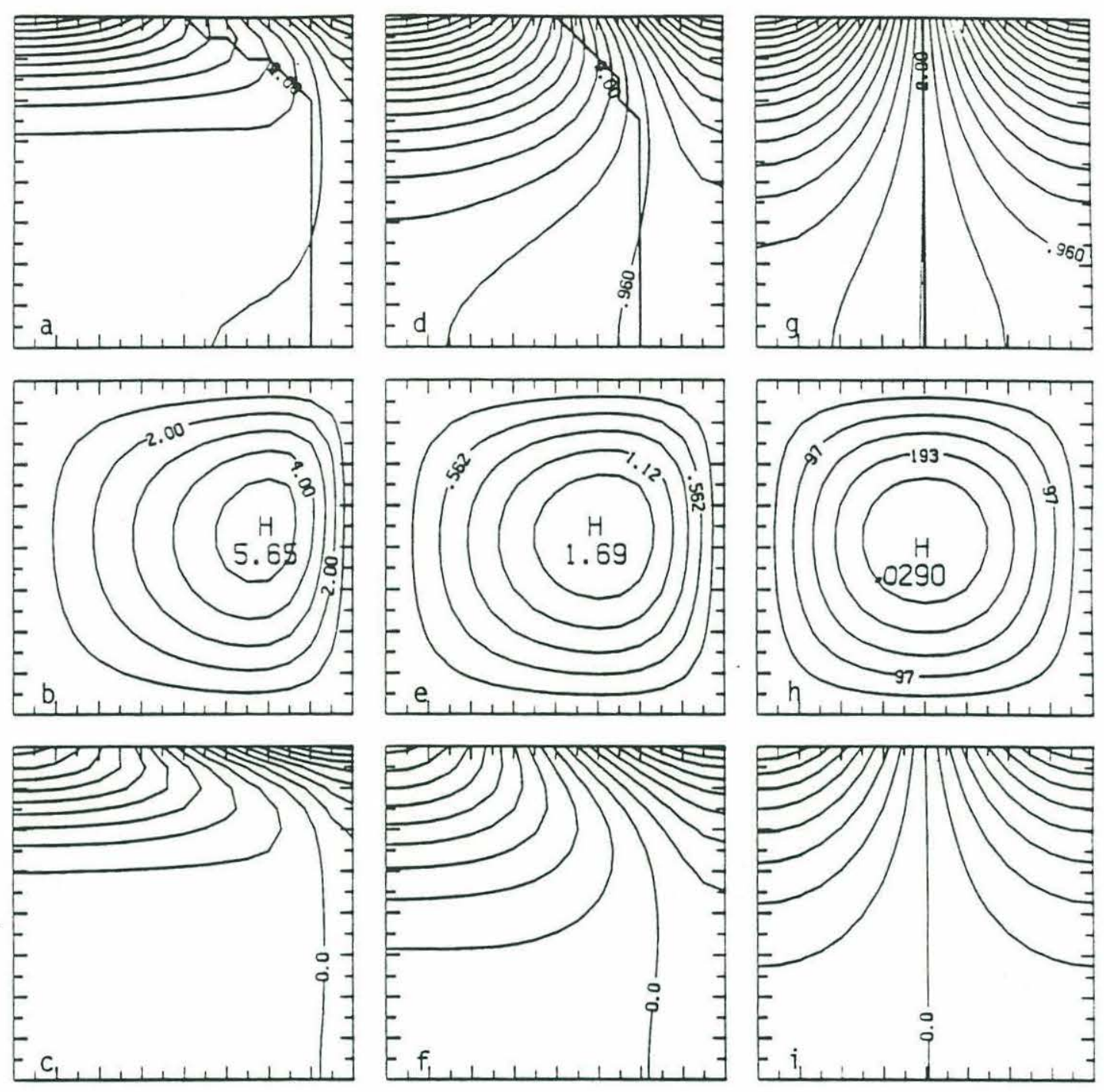

Figure 3.4: The same as figure 3.3, but for $R_{a}=2 \times 10^{5}$. 

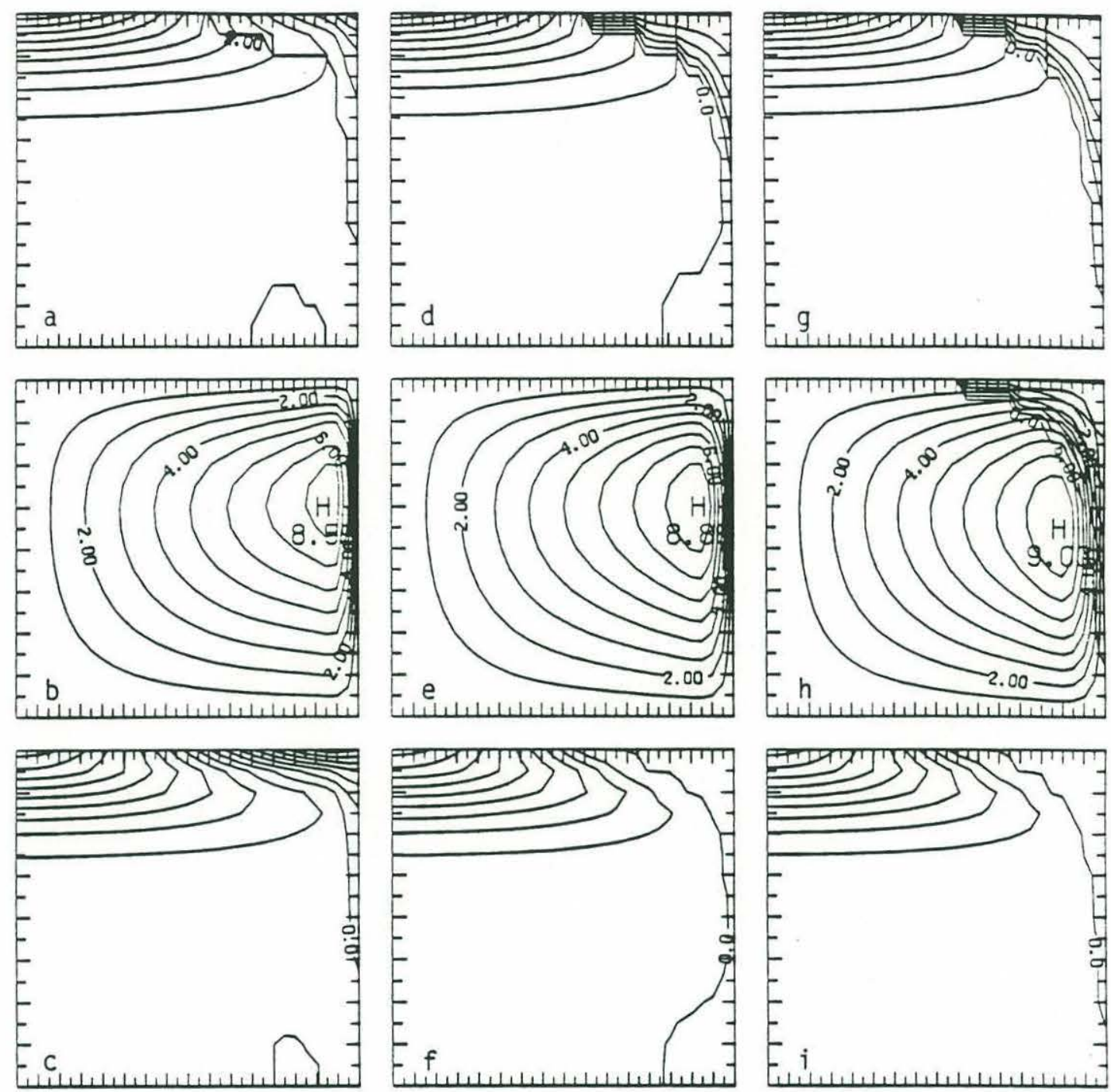

Figure 3.5: The steady state contours of $b(\mathrm{a}, \mathrm{b}$ and $\mathrm{g}), \psi(\mathrm{b}, \mathrm{e}$ and $\mathrm{h})$ and $b_{\boldsymbol{z}}(\mathrm{c}, \mathrm{f}$ and i). $R_{a} \lambda^{2}=2 \times 10^{5}$ and $\lambda=0.001$ are fixed. From left to right, the columns are for standard, density overturning and density-momentum overturning cases, respectively. The contours extending from top center to the right side wall represent $\kappa_{V}$ and $\mu_{V}$ used in the calculation. 

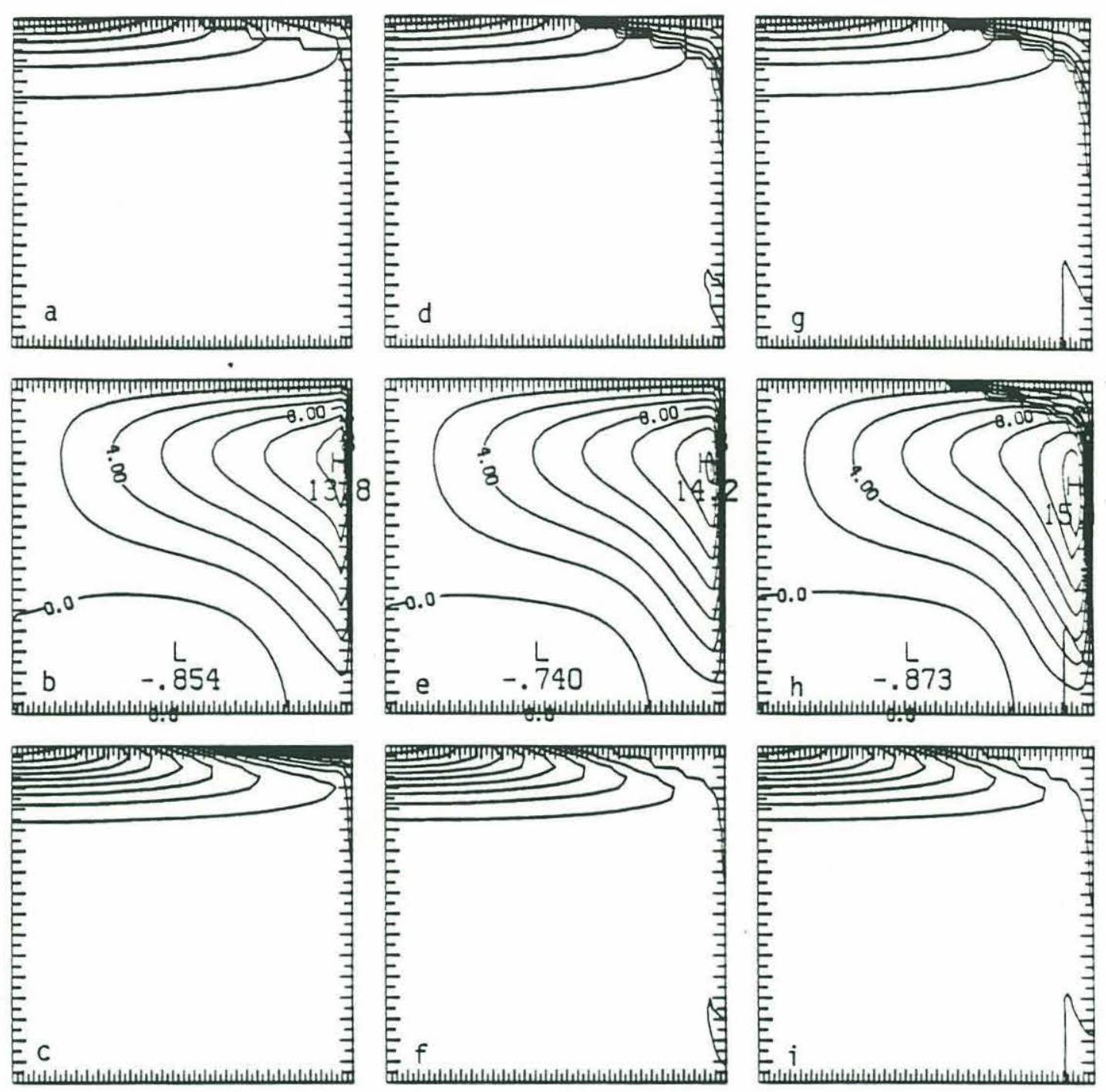

Figure 3.6: The same as figure 3.5, but for $R_{a} \lambda^{2}=2 \times 10^{6}$ and $\lambda=0.001$. 


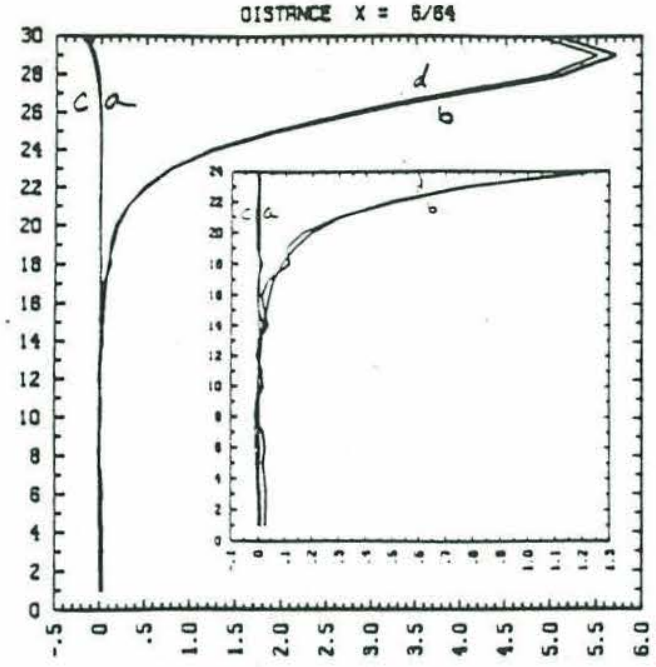

(i)

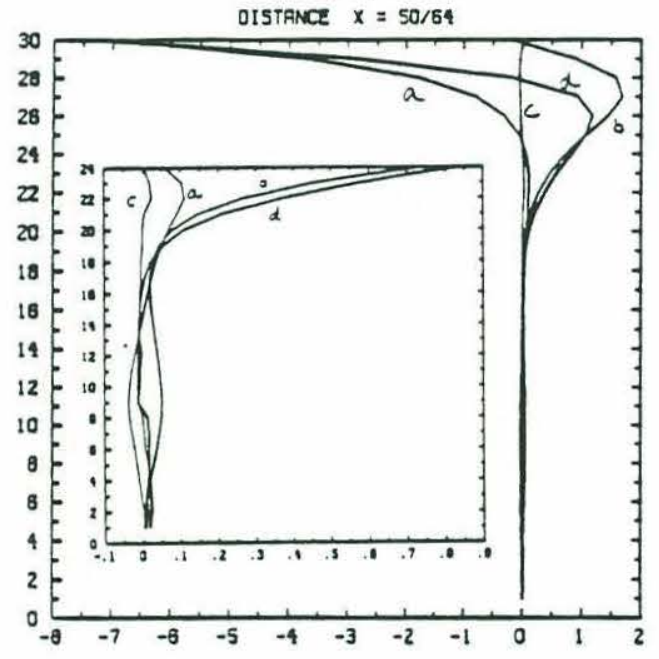

(iii)

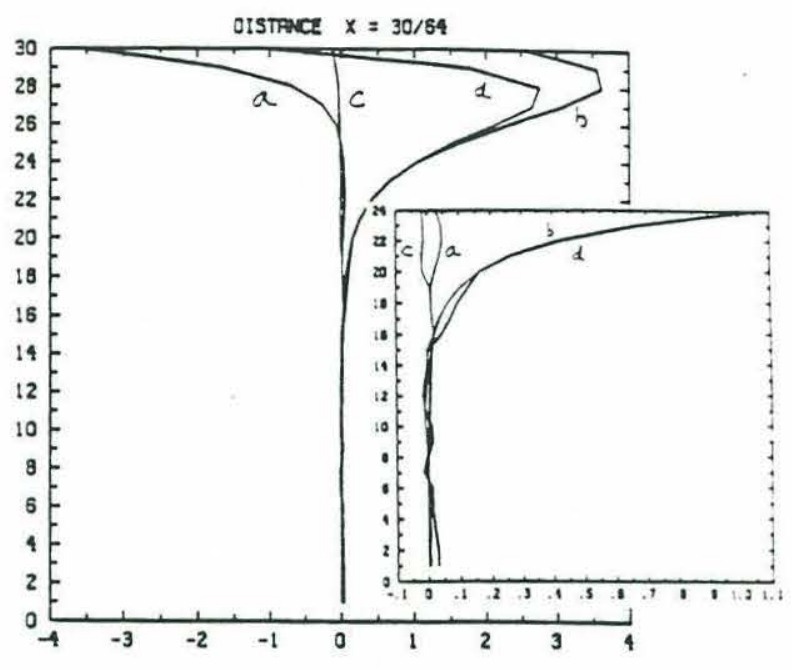

(ii)

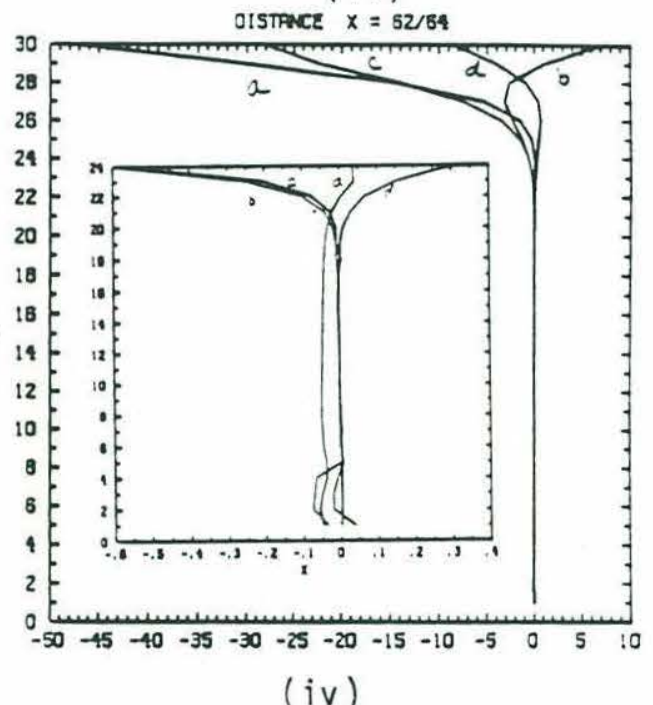

(iv)

Figure 3.7.1: The variation of the individual terms with depth in the buoyancy equation for standard case. $R_{a}=2 \times 10^{6}$ and $\lambda=0.001$. The curves in each plot are (a) $v b_{y}$, (b) $w b_{z}$, (c) $\bar{\kappa}_{H} b_{y y}$ and (d) $\left(\kappa_{V} b_{z}\right)_{z}$. The profiles are taken at four different latitudes with the inserted figure showing the variation below the thermocline. The south-north latitude values are normalized between 0 and 1 . Section plotted at latitudes (i) $6 / 64$, (ii) $30 / 64$, (iii) $50 / 64$ and (iv) $62 / 64$. 

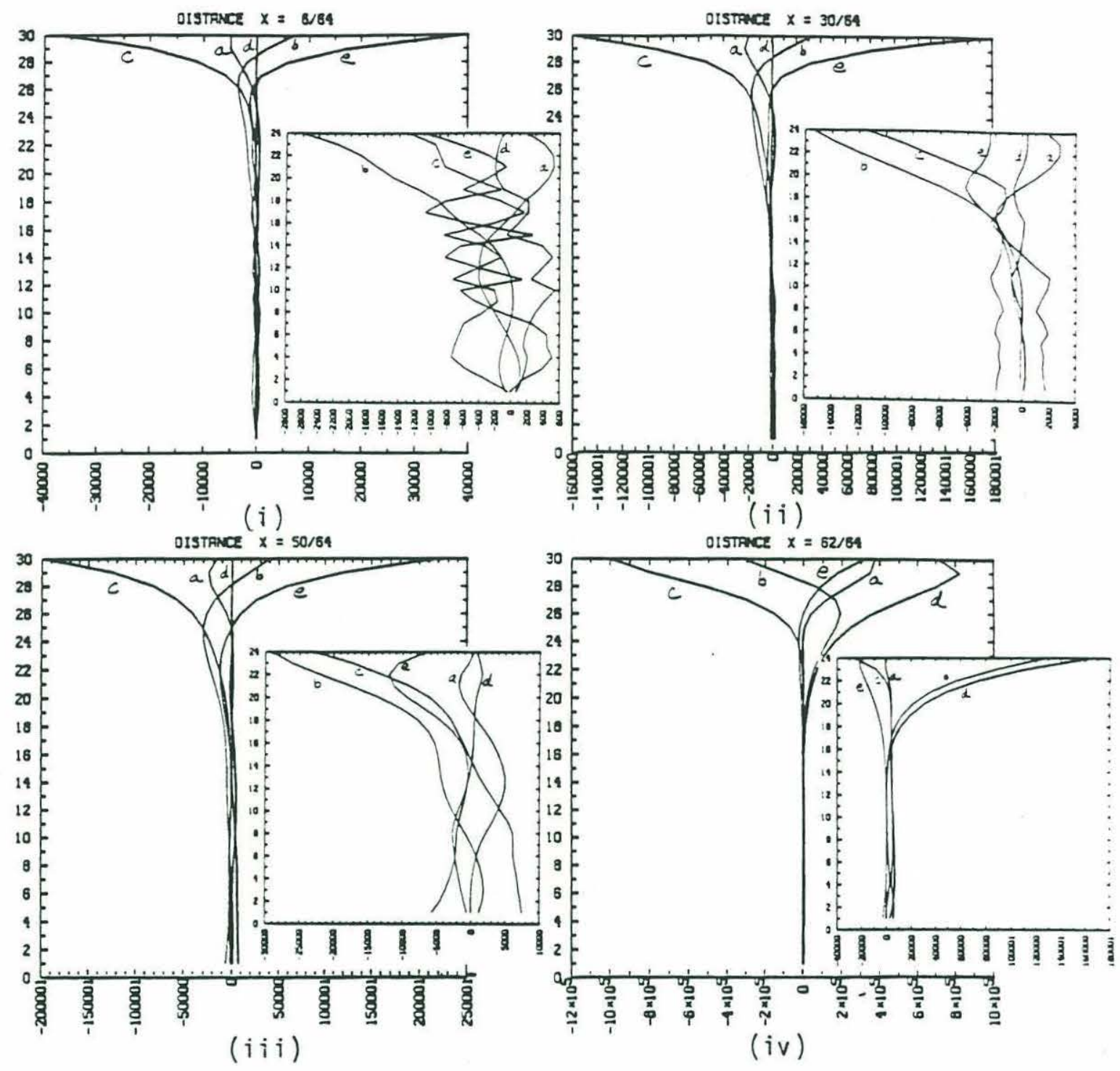

Figure 3.7.2: The variation of the individual terms with depth in the vorticity equation for standard case. $R_{a}=2 \times 10^{6}$ and $\lambda=0.001$. The curves in each plot are (a) $v\left(\nabla^{2} \psi\right)_{y}$, (b) $w\left(\nabla^{2} \psi\right)_{z}$, (c) $R_{a} \lambda^{2} b_{y}$, (d) $\bar{\mu}_{H}\left(\nabla^{2} \psi\right)_{y y}$ and (e) $\left[\nabla \cdot\left(\mu_{V} \nabla \psi_{z}\right)\right]_{z}$. The profiles are taken at four different latitudes with the inserted figure showing the variation below the thermocline. The south-north latitude values are normalized between 0 and 1 . Section plotted at latitudes (i) $6 / 64$, (ii) $30 / 64$, (iii) $50 / 64$ and (iv) $62 / 64$. 


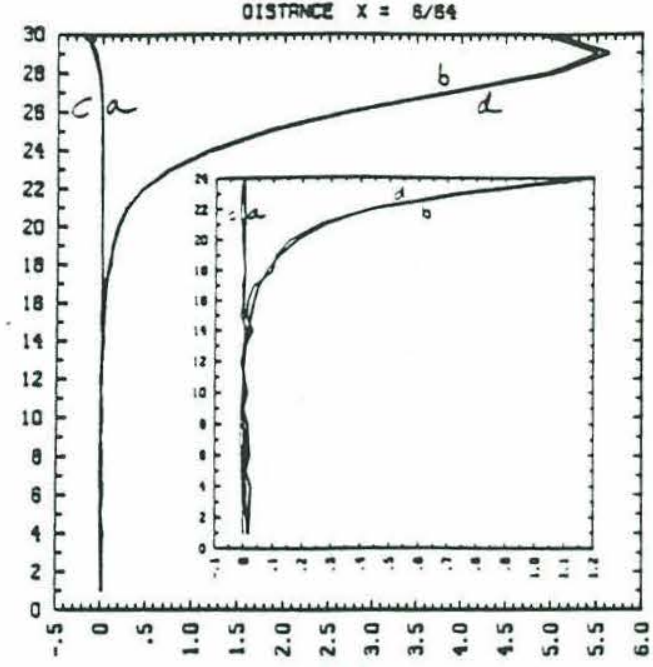

(i)

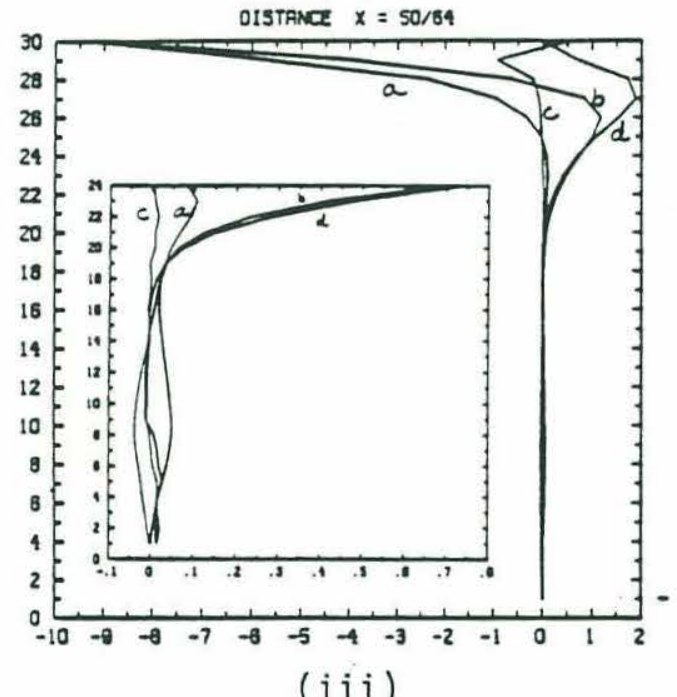

(iii)

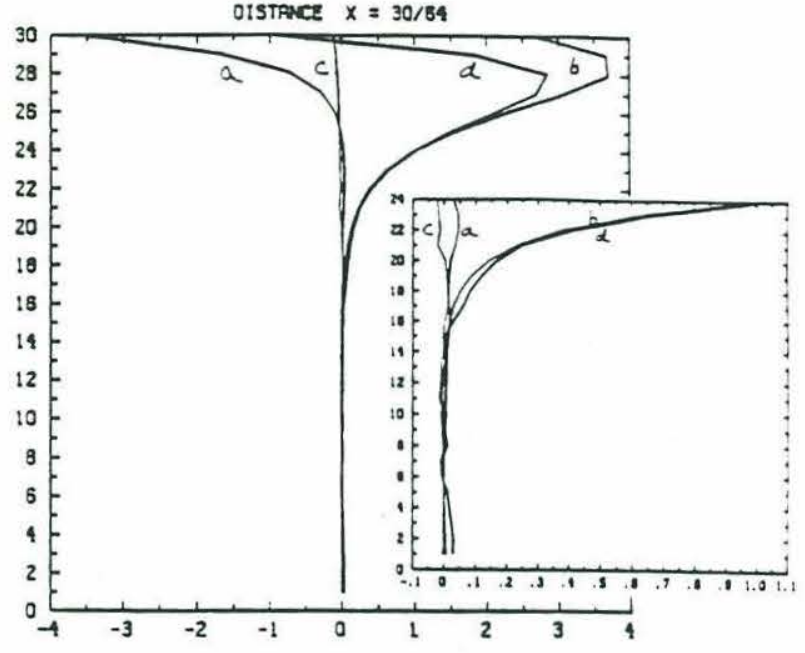

(ii)

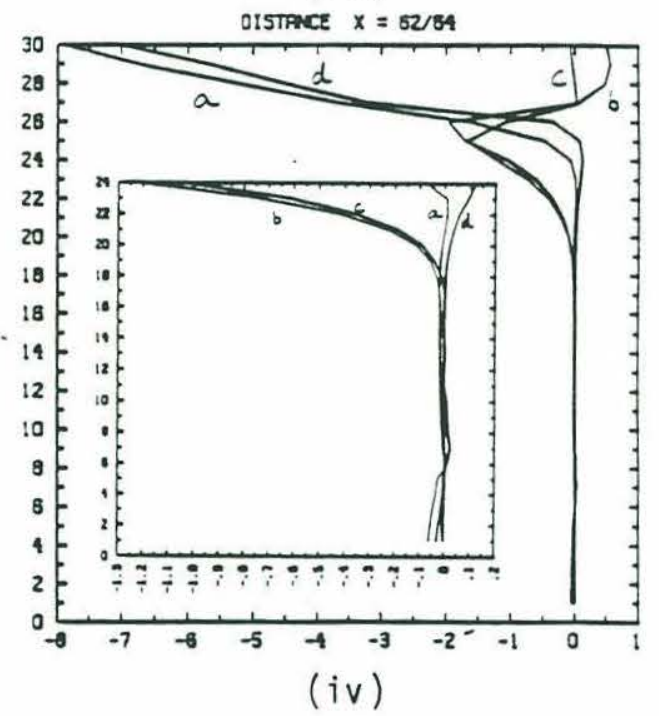

Figure 3.8.1: The same as figure 3.7.1, but for the density overturning case. 


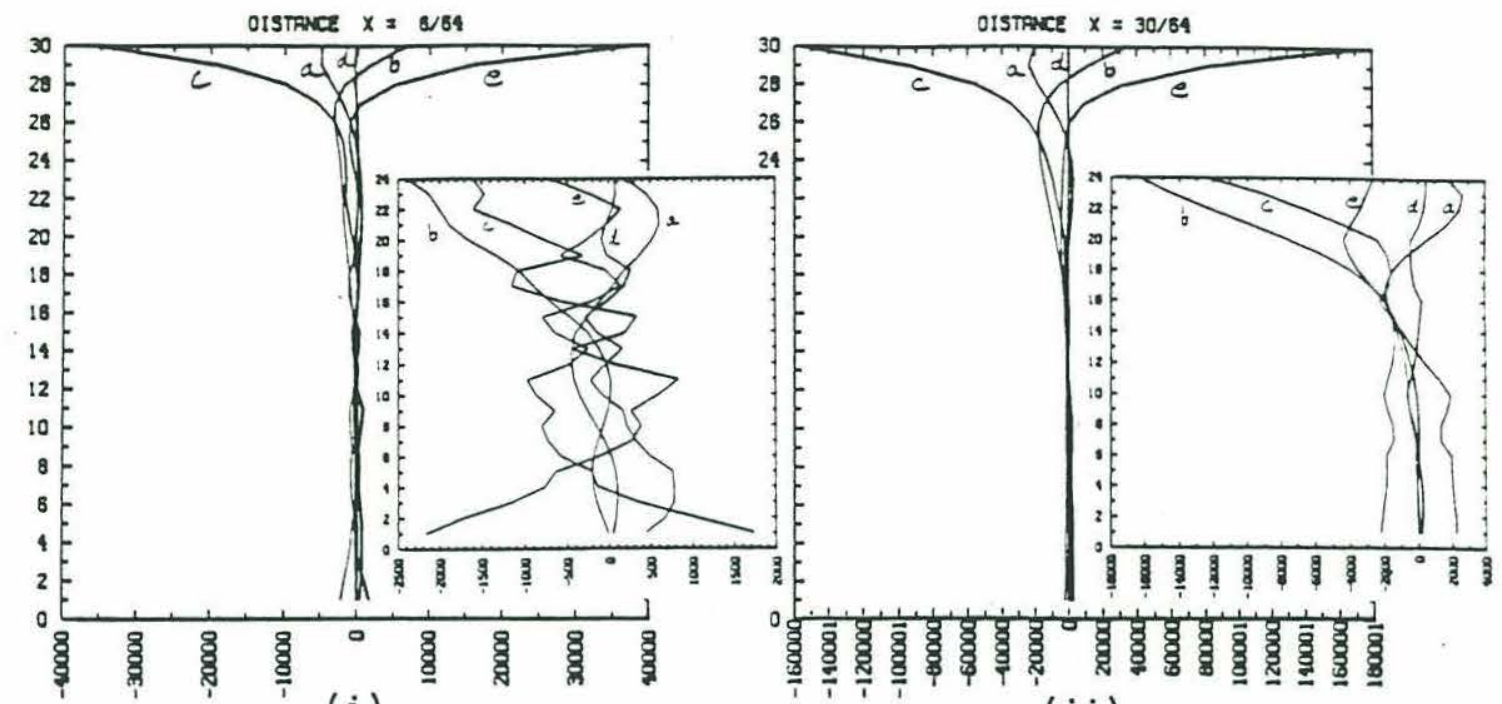

(i) $x=50 / 54$

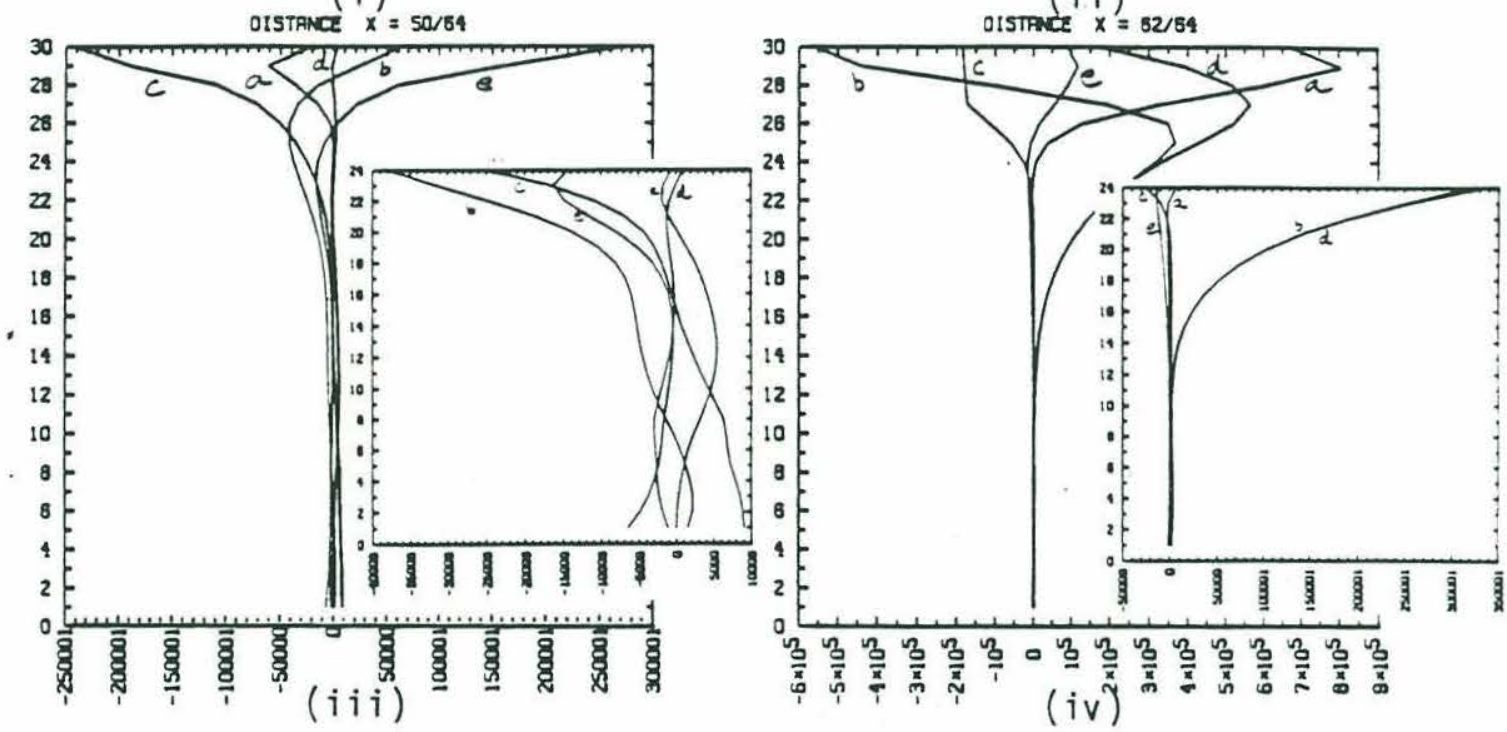

Figure 3.8.2: The same as figure 3.7.2, but for the density overturning case. 

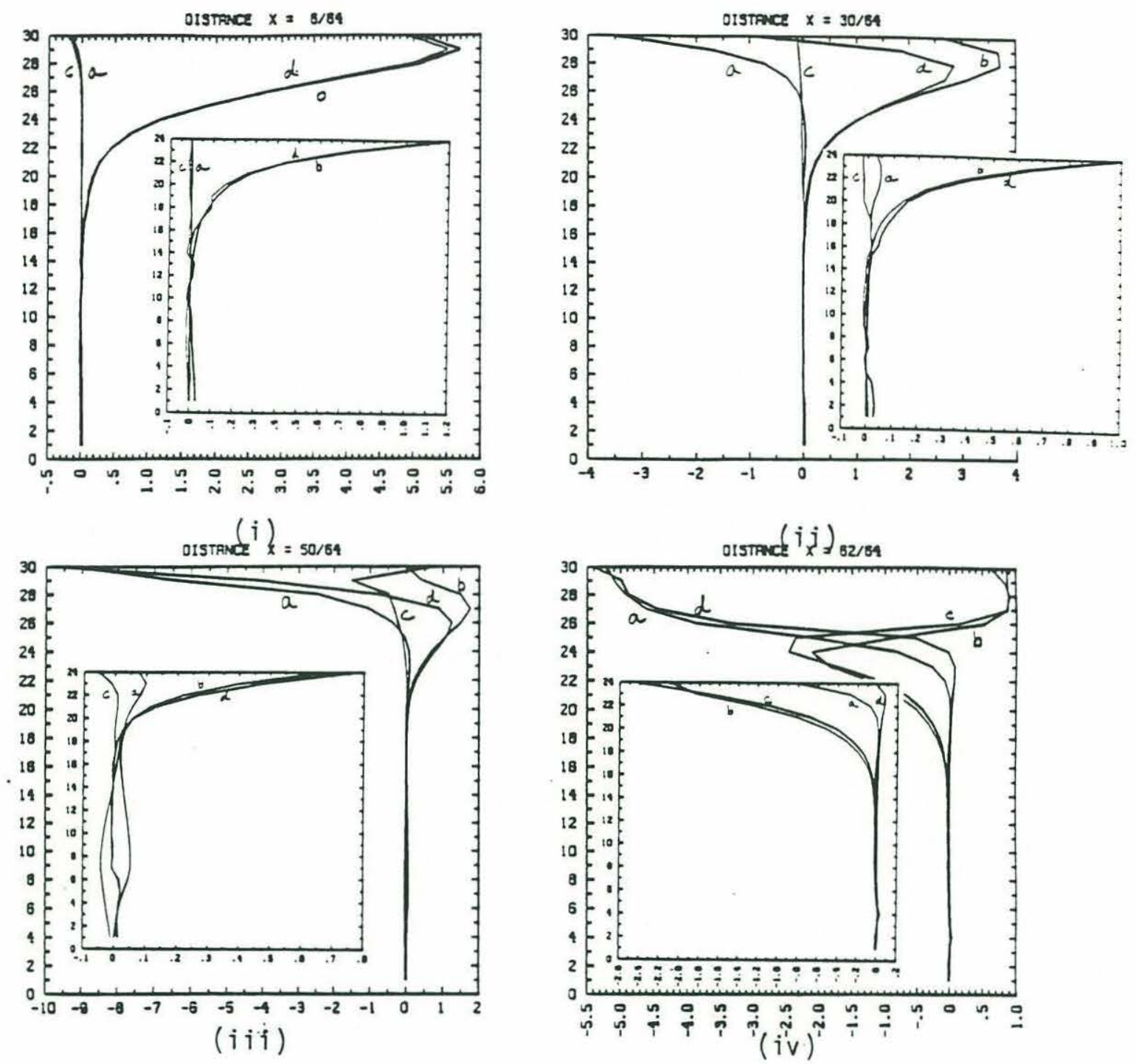

Figure 3.9.1: The same as figure 3.7.1, but for the density-momentum overturning case. 


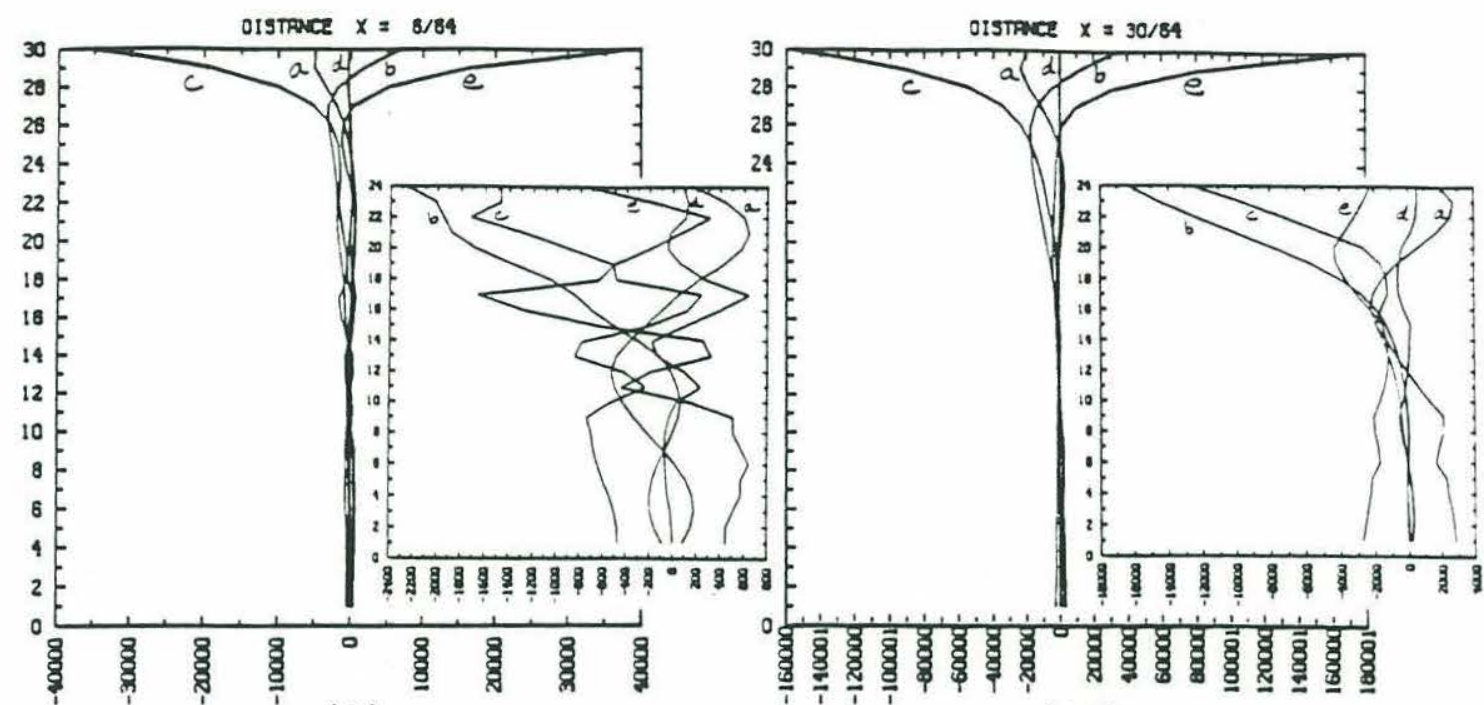

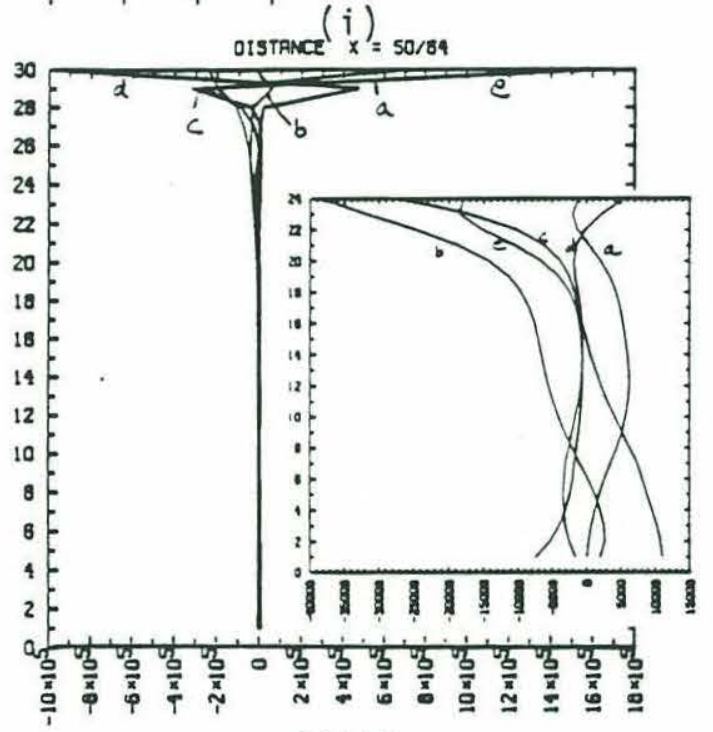

(iii)

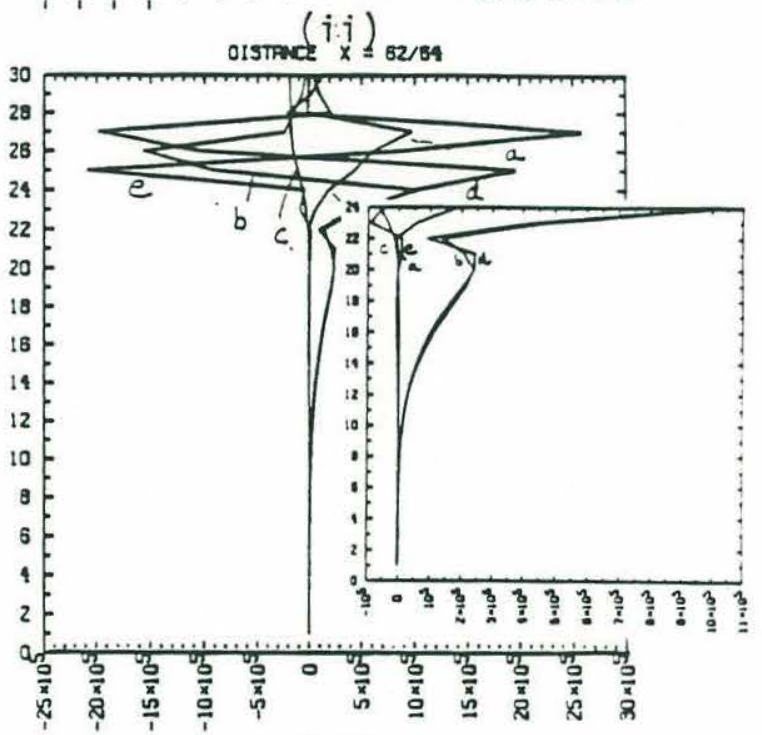

(iv)

Figure 3.9.2: The same as figure 3.7.2, but for the density-momentum overturning case. 


\section{TABLE 3.1}

\begin{tabular}{|c|c|c|c|c|c|}
\hline author & $\begin{array}{c}\text { aspect } \\
\text { ratio }\end{array}$ & range of $R_{a}$ & forcing & $\begin{array}{c}\text { boundary } \\
\text { sides }\end{array}$ & $\begin{array}{c}\text { condition } \\
\text { top/bottom }\end{array}$ \\
\hline $\begin{array}{c}\text { Barcilon } \\
\& \\
\text { Veronis }\end{array}$ & 1 & $10^{3}-10^{4}$ & $\begin{array}{c}\text { cosine } \\
\text { bottom } \\
\text { temperature }\end{array}$ & free & free/free \\
\hline $\begin{array}{c}\text { Beardsley } \\
\& \\
\text { Festa }\end{array}$ & 1 & $10^{3}-3 \times 10^{5}$ & $\begin{array}{c}\text { linear } \\
\text { bottom } \\
\text { temperature }\end{array}$ & nonslip & nonslip/nonslip \\
\hline Nelken & 1 & $2 \times 10^{4}-2 \times 10^{5}$ & $\begin{array}{c}\text { cosine } \\
\text { surface } \\
\text { flux }\end{array}$ & free & free/free \\
\hline Rossby & 0.4 & $1.1 \times 10^{7}-1.6 \times 10^{10}$ & $\begin{array}{c}\text { linear } \\
\text { bottom } \\
\text { temperature }\end{array}$ & nonslip & nonslip/nonslip \\
\hline Somerville & 0.1 & $10^{2}-10^{5}$ & $\begin{array}{c}\text { cosine } \\
\text { bottom } \\
\text { temperature }\end{array}$ & free & free/free \\
\hline Stommel & small & $R_{a} \lambda^{2} \ll 40$ & $\begin{array}{c}\text { cosine } \\
\text { surface } \\
\text { temperature }\end{array}$ & free & free/nonslip \\
\hline this study & $1-0.001$ & $2 \times 10^{4}-2 \times 10^{12}$ & $\begin{array}{c}\text { cosine } \\
\text { surface } \\
\text { flux }\end{array}$ & free & free/free \\
\hline
\end{tabular}


TABLE 3.2 $R_{a} \lambda^{2}=2 \times 10^{4}$

\begin{tabular}{|c|l|l|l|l|}
\hline aspect ratio & location of $\psi_{\max }$ & $\psi_{\max }$ & $b_{\min }$ & $b_{\max }$ \\
\hline 1 & $(11 / 17,10 / 17)$ & 3.355 & 0.814 & 1.230 \\
0.5 & $(12 / 17,10 / 17)$ & 4.116 & 0.833 & 1.224 \\
0.1 & $(14 / 17,10 / 17)$ & 4.552 & 0.850 & 1.225 \\
0.01 & $(14 / 17,10 / 17)$ & 4.536 & 0.852 & 1.225 \\
000.1 & $(14 / 17,10 / 17)$ & 4.535 & 0.852 & 1.225 \\
\hline
\end{tabular}

TABLE $3.3 R_{a} \lambda^{2}=2 \times 10^{5}$

\begin{tabular}{|c|c|c|c|c|}
\hline aspect ratio & location of $\psi_{\max }$ & $\psi_{\max }$ & $b_{\min }$ & $b_{\max }$ \\
\hline 1 & $(23 / 33,9 / 17)$ & 7.235 & 0.867 & 1.177 \\
0.5 & $(25 / 33,9 / 17)$ & 8.644 & 0.885 & 1.170 \\
0.1 & $(30 / 33,11 / 17)$ & 9.041 & 0.906 & 1.173 \\
0.01 & $(30 / 33,11 / 17)$ & 8.601 & 0.909 & 1.174 \\
000.1 & $(30 / 33,11 / 17)$ & 8.592 & 0.909 & 1.174 \\
\hline
\end{tabular}


TABLE 3.4 $R_{a}=2 \times 10^{4}$

\begin{tabular}{|c|c|c|c|c|}
\hline aspect ratio & location of $\psi_{\max }$ & $\psi_{\max }$ & $b_{\min }$ & $b_{\max }$ \\
\hline 0.5 & $(11 / 17,10 / 17)$ & 2.351 & 0.786 & 1.266 \\
0.1 & $(9 / 17,9 / 17)$ & 0.281 & 0.692 & 1.323 \\
0.01 & $(9 / 17,9 / 17)$ & 0.003 & 0.681 & 1.319 \\
\hline
\end{tabular}

TABLE 3.5 $R_{a}=2 \times 10^{5}$

\begin{tabular}{|c|c|c|c|c|}
\hline aspect ratio & location of $\psi_{\max }$ & $\psi_{\max }$ & $b_{\min }$ & $b_{\max }$ \\
\hline 0.5 & $(13 / 17,10 / 17)$ & 5.654 & 0.865 & 1.201 \\
0.1 & $(11 / 17,10 / 17)$ & 1.687 & 0.761 & 1.292 \\
0.01 & $(9 / 17,9 / 17)$ & 0.029 & 0.682 & 1.319 \\
\hline
\end{tabular}


TABLE 3.6

\begin{tabular}{|c|c|c|c|c|c|}
\hline case name & $R_{a} \lambda^{2}$ & location of $\psi_{\max }$ & $\psi_{\max }$ & $b_{\min }$ & $b_{\max }$ \\
\hline case A1 & $2 \times 10^{5}$ & $(30 / 33,11 / 17)$ & 8.592 & 0.909 & 1.174 \\
case A2 & $2 \times 10^{5}$ & $(30 / 33,11 / 17)$ & 8.859 & 0.981 & 1.173 \\
case A3 & $2 \times 10^{5}$ & $(29 / 33,10 / 17)$ & 8.995 & 0.981 & 1.172 \\
case B1 & $2 \times 10^{6}$ & $(63 / 65,25 / 33)$ & 13.845 & 0.984 & 1.123 \\
case B2 & $2 \times 10^{6}$ & $(62 / 65,25 / 33)$ & 14.249 & 0.990 & 1.122 \\
case B3 & $2 \times 10^{6}$ & $(62 / 65,23 / 33)$ & 15.519 & 0.990 & 1.122 \\
\hline
\end{tabular}




\section{Conclusions}

In this work we have treated an old problem with some new flavor. The buoyancy driven circulation in a simplified two-dimensional system is studied with the inclusion of turbulent mixing parameterization of both buoyancy and momentum and with an arbitrary aspect ratio. It is not surprising to see that the turbulent mixing homogenizes the density field and contributes to increasing the circulation. The effect of changing aspect ratio is non-intuitive and provides some insight and analogy to the oceanic problem. The combined results from this simple model study may be helpful in understanding the oceanic thermohaline circulation.

Numerical simulations have not been run at high Rayleigh number. Since tremendous difficulties in resolving boundary layers arise at high Rayleigh number, only moderate values have been used. In the past the highest Rayleigh number in numerical simulation was $3 \times 10^{5}$ (Beardsley and Festa, 1972). We have increased it to $2 \times 10^{12}$ in this study. The key to this is that we also chose a small aspect ratio. This value is still small compared to its oceanic counterpart, which is commonly $10^{17}$. But even at the present order of Rayleigh number, our model shows interesting new aspects, such as the extremely narrow northern jet layer and weak secondary circulation. It is believed that there are richer phenomina behind the increasing Rayleigh number, for example, the eventual occurrence of instability and turbulence.

This model has an advantage over the more complicated primitive equation general circulation model in its physical and mathematical simplicity. One can focus on a process and isolate it in the model without worrying about contamination or dominance by some other processes. With a properly selected physical system and solution methodology, one can gain important qualitative understanding to very complicated problem by relatively simple means. Future work should consider time dependent forcing and rotation. It will provide us with new physical insights and eventually contribute to the construction of highly realistic models of the oceanic general circulation. 


\section{References}

Arakawa, A., 1966. Computational design for long-term numerical integration of the equation of fluid motion: two-dimensional incompressible flow. Part I. Journal of Computational Physics, 1, 119-143

Arakawa, A. and V. R. Lamb, 1977. Computational design of the basic dynamical processes of the UCLA general circulation model. Methods of Computational Physics, 17, Academic Press, New York, 173-265

Barcilon, V., and G. Veronis, 1965. Thermal convection generated by differential heating along a horizontal surface. Unpublished paper, M.I.T.

Beardsley, R. C., and J. Festa, 1972. A numerical model of convection driven by a surface stress and a non-uniform horizontal heating. Journal of Physical Oceanography, 2, 444-455

Bryan, K., and M. D. Cox, 1968. A nonlinear model of an ocean driven by wind and differential heating: Part I. Description of the three dimensional velocity and density fields. Journal of the Atmospheric Sciences, 25, 945-967

Buneman, O., 1969. A compact non-iterative Poisson solver.SU-IPR, 294, 1-11

Chandrasekhar, S., 1961. Hydrodynamic and hydromagnetic stability. Oxford University Press, 220-271, 652pp

Killworth, P. D., 1976. The mixing and spreading phase of MEDOC. I. Progress in Oceanography, 7, 59-90

Nelken, H., 1987. Thermally Driven Circulation. Sc.D. Thesis. MIT/WHOI, WHOI87-33

Press, W. H., etc., 1986. Numerical Recipes. Cambridge University Press. 818pp

Roache, P. J., 1972. Computational Fluid Dynamics. Hermosa Publishers. 446pp

Rossby, H. T., 1965. On the thermal convection driven by non-uniform heating from below, an experimental study. Deep-Sea Research, 12, 9-16

Somerville, C. J. R., 1967. A nonlinear spectral model of convection in a fluid unevenly heated from below. Journal of the Atmospheric Sciences, 24, 665-676

Stommel, H., 1950. An example of thermal convection. Transection of the American Geophysical Union, 31, 553-554

Stommel, H., 1962. On the smallness of sinking region in the ocean. Proceedings of the National Academy of science, U.S., 48, 766-772

Stone, P. H., 1968. Some properties of Hadley regimes on rotating and non-rotating planets. Journal of the Atmospheric Sciences, 25, 644-657

Woods, J. D., 1973. Space-time characteristics of turbulence in the seasonal thermocline. Proceedings of the Hydrodynamics Colloquium, liege, Belgium 